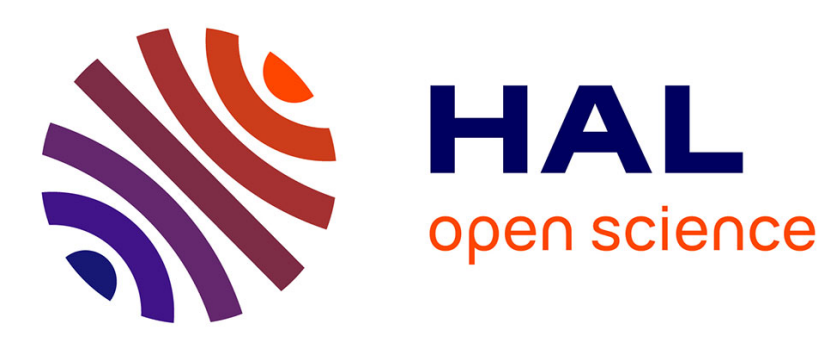

\title{
Fluid/Structure Coupled Aeroelastic Computations for Transonic Flows in Turbomachinery
}

\author{
Hirofumi Doi
}

\section{To cite this version:}

Hirofumi Doi. Fluid/Structure Coupled Aeroelastic Computations for Transonic Flows in Turbomachinery. Engineering Sciences [physics]. Stanford university, 2002. English. NNT: . tel-01333735

\section{HAL Id: tel-01333735 \\ https://theses.hal.science/tel-01333735}

Submitted on 25 Jun 2016

HAL is a multi-disciplinary open access archive for the deposit and dissemination of scientific research documents, whether they are published or not. The documents may come from teaching and research institutions in France or abroad, or from public or private research centers.
L'archive ouverte pluridisciplinaire HAL, est destinée au dépôt et à la diffusion de documents scientifiques de niveau recherche, publiés ou non, émanant des établissements d'enseignement et de recherche français ou étrangers, des laboratoires publics ou privés. 


\section{FLUID/STRUCTURE COUPLED AEROELASTIC COMPUTATIONS FOR TRANSONIC FLOWS IN TURBOMACHINERY}

Hirofumi Doi

August 1st, 2002

A dissertation submitted to the department of aeronautics and astronautics and the committee on graduate studies of stanford university in partial fulfillment of the requirements for the degree of doctor of philosophy

\section{committee members}

Juan J. Alonso

Antony Jameson

Holt Ashley

Roger L. Davis 


\title{
FLUID/STRUCTURE COUPLED AEROELASTIC COMPUTATIONS FOR TRANSONIC FLOWS IN TURBOMACHINERY
}

\author{
A DISSERTATION \\ SUBMITTED TO THE DEPARTMENT OF AERONAUTICS AND ASTRONAUTICS \\ AND THE COMMITTEE ON GRADUATE STUDIES \\ OF STANFORD UNIVERSITY \\ IN PARTIAL FULFILLMENT OF THE REQUIREMENTS \\ FOR THE DEGREE OF \\ DOCTOR OF PHILOSOPHY
}

Hirofumi Doi

August 2002 
(C) Copyright by Hirofumi Doi 2002

All Rights Reserved 
I certify that I have read this dissertation and that in my opinion it is fully adequate, in scope and quality, as a dissertation for the degree of Doctor of Philosophy.

Juan J. Alonso
(Principal Adviser)

I certify that I have read this dissertation and that in my opinion it is fully adequate, in scope and quality, as a dissertation for the degree of Doctor of Philosophy.

\section{Antony Jameson}

I certify that I have read this dissertation and that in my opinion it is fully adequate, in scope and quality, as a dissertation for the degree of Doctor of Philosophy.

\section{Holt Ashley}

I certify that I have read this dissertation and that in my opinion it is fully adequate, in scope and quality, as a dissertation for the degree of Doctor of Philosophy.

Roger L. Davis

(University of California, Davis)

Approved for the University Committee on Graduate Studies: 


\section{Abstract}

The unstable, self-excited or forced vibrations of rotor blades must be avoided in designing high performance turbomachinery components because they may induce catastrophic structural failures. In evaluating the stability of such vibrations, computational approaches have been bearing an increasing role due to the surprising progress of both computer technologies and advanced algorithms. They are now at a stage where time domain fluid/structure coupled simulations of aeroelastic phenomena in turbomachinery with realistic geometries can be used in practice. The present study demonstrates the capabilities of a fluid/structure coupled computational approach which consists of an unsteady three-dimensional Navier-Stokes flow solver, $T F L O$, a finite element structural analysis package, $M S C / N A S T R A N$, and the coupling interface between the two disciplines. The flow solver relies on a multiblock, cell-centered finite volume discretization and the dual time stepping time integration scheme with multigrid for convergence acceleration. Parallelization for multiple processors is also performed to achieve faster computations making use of the Message Passing Interface (MPI). As far as the interface is concerned, high accuracy is pursued with respect to load transfer, deformation tracking and synchronization. As a result, the program successfully predicts the aeroelastic responses of a high performance fan, NASA Rotor $6^{7}$, over a range of operational conditions. The major contribution to the aerodynamic damping for turbomachinery blade motions is observed to be the unsteady pressure generated at the location of the shock. The results show that the 
unsteady pressure may act to damp or excite the blade motion mainly depending on the inter-blade phase angle. It is concluded that the level of fidelity in the individual disciplines, together with an accurate coupling interface will allow for accurate prediction of flutter boundaries of turbomachinery components. 


\section{Acknowledgments}

I would like to express my deepest gratitude to my advisor, Professor Juan Jose Alonso for suggesting this project and providing guidance throughout the course of this thesis. He has given me considerable freedom and shown patience as I pursued the idea development in this project. I would also like to thank Professor Roger L. Davis for many fruitful suggestions and for providing me with the computational grid for Rotor 67. I would further like to thank the readers, Professor Antony Jameson and Professor Holt Ashley, for taking the time to read this thesis, and making valuable suggestions.

I thank my fellow students for useful discussions with them and various help including computer support and my priority in using computer resources. I thank the people involved in the TFLO development program for providing me with the upto-date sophisticated solver. I thank my friends for their encouragement which has been a vital contribution to this work. I would also like to acknowledge the financial support provided by the Japan Defense Agency. 


\section{Contents}

Abstract $\quad$ iv

Acknowledgments vi

1 Introduction $\quad 1$

1.1 Description of the Problem . . . . . . . . . . . . . . . 1

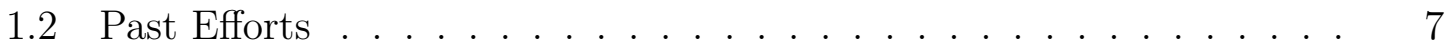

1.2.1 Linearized Unsteady Aerodynamic Theory . . . . . . . . . 7

1.2.2 Time-Linearized Computations for Unsteady Aerodynamics . 11

1.2.3 Time-Marching Computations for Unsteady Aerodynamics . . 12

1.2.4 Fluid/Structure Coupled Computations . . . . . . . . . . . 15

1.3 Motivations of the Research . . . . . . . . . . . . . . 17

2 Governing Equations $\quad 20$

2.1 Unsteady Aerodynamics . . . . . . . . . . . . . . . . . . . 21

2.1.1 Reynolds' Transport Theorem . . . . . . . . . . . . . . . 22

2.1 .2 Conservation of Mass . . . . . . . . . . . . . . . . . 22

2.1.3 Conservation of Momentum . . . . . . . . . . . . . . 23

2.1.4 Conservation of Energy . . . . . . . . . . . . . . . 25

2.1.5 The Reynolds-Averaged Navier Stokes Equations _. . . . . 27

2.1.6 Conservation Law Form _. . . . . . . . . . . . . 29

2.2 Structural Mechanics . . . . . . . . . . . . . . . . . . . 31

2.2.1 Differential Equations of Elasticity _ . . . . . . . . 32

2.2 .2 The Principle of Virtual Work . . . . . . . . . . . . . . . 34 
2.3 Fluid/Structure Interaction $\ldots \ldots \ldots \ldots$

2.3.1 Conservation of Loads and Energy _ . . . . . . . . . . 35

2.3.2 Geometric Conservation Law . . . . . . . . . . . . . . . . 36

3 Description of the Method $\quad 39$

3.1 Unsteady Aerodynamics . . . . . . . . . . . . . . . . . . . 40

3.1.1 Cell-Centered Finite Volume Scheme . . . . . . . . . . . . 43

3.1 .2 Artificial Dissipation . . . . . . . . . . . . . . 45

3.1 .3 Dual Time Stepping . . . . . . . . . . . . . . . 47

3.1.4 Time Marching Scheme . . . . . . . . . . . . . . . 50

3.1 .5 Multigrid . . . . . . . . . . . . . . . . 52

3.1 .6 Boundary Conditions . . . . . . . . . . . . . . . 54

3.1.7 Turbulence Model . . . . . . . . . . . . . . . . . . . . . . . . . 61

3.1 .8 Moving Mesh System . . . . . . . . . . . . . . . . . . . 64

3.2 Structural Mechanics . . . . . . . . . . . . . . . . . . . . 71

3.2.1 Finite Element Analysis _ . . . . . . . . . . . . . . 72

3.2 .2 Finite Element Model _. . . . . . . . . . . . . . . . . . 75

3.2 .3 Damping Characteristics . . . . . . . . . . . . . 76

3.2.4 Centrifugal and Coriolis Forces _. . . . . . . . . 78

3.2 .5 Time Integration $\ldots \ldots \ldots$. . . . . . . . . . . 81

3.3 Fluid-Structure Interface . . . . . . . . . . . . . . . . . 83

3.3.1 Deformation Tracking System . . . . . . . . . . . . . 84

3.3.2 Load Transfer System _. . . . . . . . . . . . . . . . . 88

3.3 .3 Synchronization . . . . . . . . . . . . . . . . . . 90

3.4 Parallelization . . . . . . . . . . . . . . . . . . . . 93

3.4 .1 Flow Solver . . . . . . . . . . . . . . . . . . . . . . . 94

3.4 .2 Structural Solver . . . . . . . . . . . . . . . . . 96

$\begin{array}{lll}4 & \text { Results } & 97\end{array}$

4.1 Code Evaluation - Cantilever Double Circular Arc Airfoil Wing . . . 97

4.2 Flutter Analysis Demonstration - NASA Rotor 67 . . . . . . . . 105

4.2 .1 NASA Rotor $67 \ldots \ldots \ldots$. . . . . . . . . . . 105 
4.2.2 Computational Grid . . . . . . . . . . . . . . . . 108

4.2.3 Blade Structural Model . . . . . . . . . . . . . . . . . . . . 112

4.2 .4 Steady Flow . . . . . . . . . . . . . . . . . 115

4.2.5 Structure-Coupled Unsteady Flow . . . . . . . . . . . . . . 121

5 Conclusions and Future Work $\quad 140$

$\begin{array}{ll}\text { Bibliography } & 143\end{array}$ 


\section{List of Tables}

4.1 Material Properties . . . . . . . . . . . . . . . . . . . . . 113

4.2 Case Matrix for Rotor 67 Aeroelastic Calculations . . . . . . . . . . 125 


\section{List of Figures}

1.1 Flutter Boundaries on a Compressor Map . . . . . . . . . . . . 2

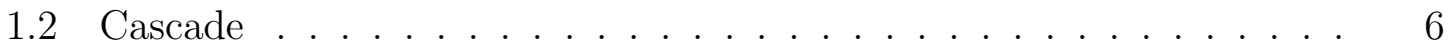

3.1 Fluid/Structure Coupling _. . . . . . . . . . . . . . . . 41

3.2 Control Volume . . . . . . . . . . . . . . . . . . . . . . . . 44

3.3 Multigrid Cycle . . . . . . . . . . . . . . . . . . . . 55

3.4 Halo Cells and Boundary Condition on Solid Body Surfaces . . . . 56

3.5 Computational Domain and Boundary Conditions for Turbomachinery 56

3.6 Moving Mesh Procedure . . . . . . . . . . . . . . 66

3.7 Moving Mesh Procedure in the Tip Clearance Region . . . . . . . . 69

3.8 Perturbed Mesh for the Tip Clearance Using the Usual Procedure . . 70

3.9 Perturbed Mesh for the Tip Clearance Using the Improved Procedure 70

3.10 Deformation Tracking System . . . . . . . . . . . 86

3.11 Load Transfer System . . . . . . . . . . . . . . . . 90

3.12 Simple Partitioned Stagger Procedures _. . . . . . . . . . . . 92

4.1 Cantilever Double Circular Airfoil Wing . . . . . . . . . . 98

4.2 Mode Shapes of the Cantilever Double Circular Arc Airfoil Wing . . . 99

4.3 Computational Grid for the Cantilever Double Circular Arc Airfoil Wing 101

4.4 Time History of the Displacements at the Mid-Chord of the Tip . . 102

4.5 Dynamic Pressure at the Flutter Onset for Various Mach Number . . 104

4.6 NASA Rotor $67 \ldots \ldots$. . . . . . . . . . . . . 107

4.7 Meridional View of Computational Grid for NASA Rotor 67 . . . . 109

4.8 Blade-to-Blade View of Computational Grid for NASA Rotor 67 . . . 110 
4.9 Grid Near the Tip Clearance . . . . . . . . . . . . . . . . . . . . 111

4.10 Finite Element Model of the NASA Rotor 67 . . . . . . . . . . . . . 112

4.11 Mode Shape of the Rotor 67 . . . . . . . . . . . . . . . . . 114

4.12 Comparison of Rotor Performance at Design Speed . . . . . . . . . . 117

4.13 Experimental and Numerical Relative Mach Number Contour for Near Peak Efficiency . . . . . . . . . . . . . . . . . 119

4.14 Flow Variable Distributions in the Span-Wise Direction at the Downstream of the Blade for Near Peak Efficiency . . . . . . . . . . . . . . 120

4.15 Experimental and Numerical Relative Mach Number Contour for Near

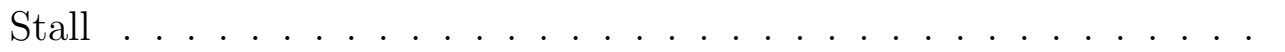

4.16 Flow Variable Distributions in the Span-Wise Direction at the Downstream of the Blade for Near Stall . . . . . . . . . . . . . . . . . 123

4.17 Deflection at the Mid-Chord of the Tip Section for Near Peak Effi-

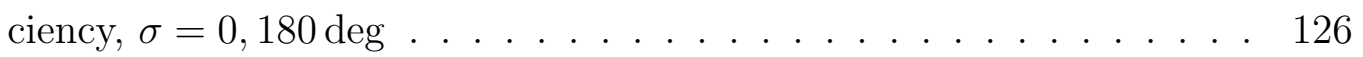

4.18 Deflection at the Mid-Chord of the Tip Section for Near Peak Effi-

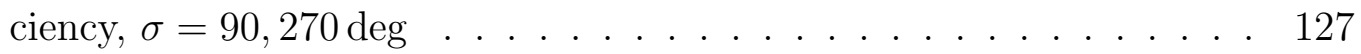

4.19 Unsteady Pressure Distribution on the Blade Surfaces for Near Peak

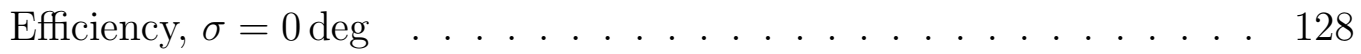

4.20 Unsteady Pressure Distribution on the Pressure Side for Near Peak

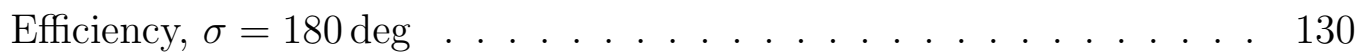

4.21 Work per Cycle Distribution on the Blade Surface for Near Peak Efficiency, $\sigma=0$ deg . . . . . . . . . . . . . . . . . . . . . . . 132

4.22 Work per Cycle Distribution on the Blade Surface for Near Peak Effi-

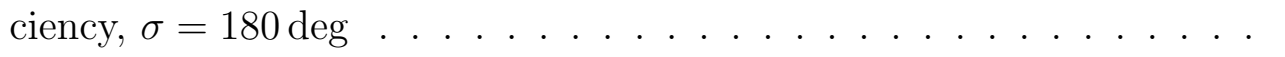

4.23 Deflection at the Mid-Chord of the Tip Section for Near Stall, $\sigma=$

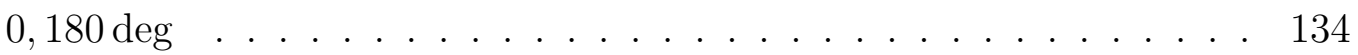

4.24 Work per Cycle Distribution on the Blade Surface for Near Stall, $\sigma=$ $0,180 \mathrm{deg} \ldots \ldots \ldots \ldots \ldots \ldots \ldots$

4.25 Experimental and Numerical Relative Mach Number Contour for Near

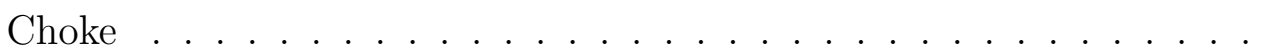


4.26 Deflection at the Mid-chord of the Tip Section for Near Choke, $\sigma=$ $0,180 \operatorname{deg} \ldots \ldots \ldots \ldots \ldots \ldots \ldots \ldots \ldots$

4.27 Work per Cycle Distribution on the Blade Surface for Near Choke, $\sigma=0,180 \operatorname{deg} \ldots \ldots \ldots \ldots \ldots \ldots \ldots$ 


\section{Chapter 1}

\section{Introduction}

\subsection{Description of the Problem}

In the operation of jet engines, the aeroelastic behavior of blades in fans and compressors related to phenomena such as inlet flow distortion, blade row interactions, flutter and limit cycle oscillations, could induce not only high cycle fatigue but also structural failure of the blades and, possibly, extensive damage to the engine with catastrophic consequences to the aircraft for which these engines provide the necessary thrust. The vibrations leading to such failures can be stable, as in the case of forced vibrations from inlet distortions or blade row interactions, or they can be unstable, as is the case of self-excited vibrations or flutter. Because of the close interaction between performance and structural integrity, designers of jet engines must place great importance on aeroelastic effects to optimize a given design. However, they must also pursue higher engine performance by reducing the blade thickness and weight, which has a negative impact on the aeroelastic behavior of the blades. As a result of the pursuit of high performance, the dynamic operating line during transients such as accelerations and decelerations, may intersect the flutter boundaries in the characteristic map shown in Figure1.1. This figure represents the overall characteristics 


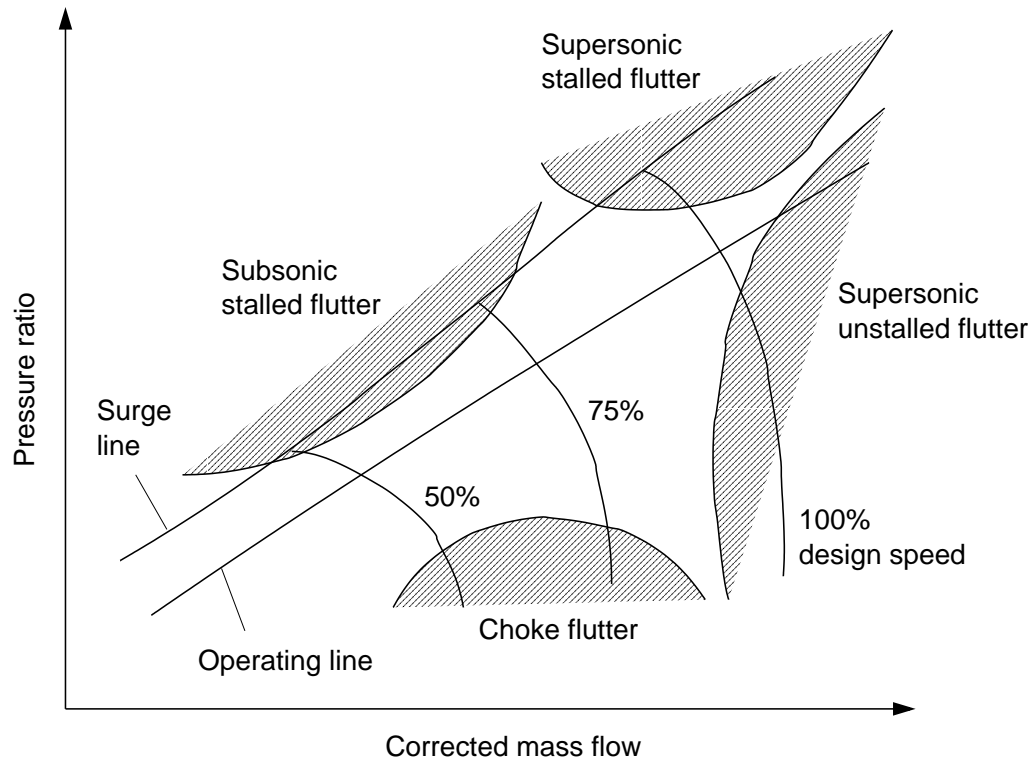

Figure 1.1: Flutter Boundaries on a Compressor Map

of a complete multi-stage compressor made up of a number of sequential stages. An important property of the compressor map is the fact that each point corresponds theoretically to a unique value for angle of attack and Mach number at any reference airfoil section in the compressor.

The aeroelastic behavior known as flutter depends on the point of operation on the characteristic compressor map [1]. Common types of flutter have been designated as unstalled supersonic flutter, subsonic/transonic stalled flutter, supersonic stalled flutter, and choke flutter. Supersonic flutter can occur on the operating line in Figure 1.1 on which the engine is designed to operate; the other types of flutter occur at off-design conditions despite being inside the surge line.

Very early in the history of turbomachinery development, rotor blades were found to experience severe vibrations at part-speed operation. Diagnosis revealed that they were in fact subsonic stalled flutter oscillations. Fortunately, the region of subsonic stalled flutter is above the operating line on the compressor map in Figure 1.1 and 
extends up to the surge line. The seriousness of the problem is, however, stressed by the fact that the conditions for this type of flutter could be achieved at ground conditions and re-entered during high speed flight at low altitude. Typically subsonic stalled flutter occurs at part-speed operation and is confined to usually the first two or three rotor stages operating at higher than average incidence [2]. Naturally, the stalled tip of a rotor blade must extract energy from the fluid flow resulting in a pitching vibration of airfoil sections near the tip. On the other hand, when considering threedimensional effects, the energy of the vibration is put back into the fluid flow by airfoil sections at smaller radii and that is dissipated from the system by damping. The stalled flutter occurs when the extracted energy is larger than the energy put back into the fluid. These effects usually make the mode shape of this kind of flutter the first torsional mode of the blade.

In the middle stages of a multi-stage compressor it may be possible to enter another region on the compressor map in Figure 1.1, where so-called choke flutter appears. This type of flutter normally occurs at part-speed operation and is confined to those rotor stages operating at lower than average incidence, where possibly negative values of incidence are encountered. This region of flutter normally lies below the usual operating line on a compressor map. This type of instability is related to the compressibility of the fluid and separation of the flow is typically involved. Presumably, separation at the leading edge of each blade on the pressure surface, and the relative motion between adjacent blades as they vibrate, conspire to change the effective throat location of the flow passage in a time dependent manner [2]. Those oscillatory changes affect the pressure distribution on each blade in such a way as to pump energy from the fluid into the blade vibration and thus sustain or amplify the motion. However the exact nature of the choke flutter mechanism is still controversial.

Similarly to subsonic stalled flutter, supersonic stalled flutter occurs at higher 
compressor pressure ratios, above the operating line, but near the 100 percent design speed. In this region, stalling of the flow is involved since the region is in the neighborhood of the surge limit line. Since the blades are operating at relatively high positive incidences in a supersonic inflow, there is a detached bow shock at the entrance of each blade passage as a consequence of maintaining a high pressure ratio. The motion of the impinging shock wave originating from the leading edge of the adjacent blade may cause flutter in the first bending mode associated with a certain inter-blade phase angle [3]. However the mechanism is still uncertain because little research has been done on this type of flutter.

Supersonic unstalled flutter, in either the torsion or bending mode, is usually encountered along the operating line, and then only at corrected over-speed conditions, in which large fan rotors tips are operating at supersonic Mach numbers. The boundary of this type of flutter is typically so close to the operating line at or near take-off power that it is taken as practically the most serious kind of flutter. It is said that there are two noticeable characteristics of supersonic unstalled flutter [4] that do not strongly appear in subsonic flutter. The first feature is a strong blade loading effect, which makes the boundary, i.e., the flutter onset rotation speed, change according to the pressure ratio, as shown in Figure 1.1. The second feature is the rapid increase of oscillatory motion observed when the boundary is crossed. Within the blade passage, shock waves are formed in various ways determined by the blade geometry, the Mach number and the pressure ratio. The unsteady aerodynamic forces resulting from these shock movements have a pronounced effect on flutter boundaries. Bendiksen [5] showed that both stabilizing and destabilizing effects are observed, depending on the shock structure and the vibrational inter-blade phase angle, which will be discussed later. In his work, it is implied that the flutter boundaries lie on the point on the compressor map corresponding to the change of shock structures, which are driven by the change of back pressure arising from the change of pressure ratio or rotor speed. 
He also suggests that the increase of back pressure, in other words, the decrease of Mach number, should stabilize torsional oscillations and destabilize bending oscillations in general. On the other hand, the increase of Mach number strengthens the shock wave and pushes the shock reflection point on the adjacent blade aft, which would help torsional oscillations with a certain inter-blade phase angle.

In addition to the Mach number and the dynamic pressure, which govern the stability of isolated wing flutter, the inter-blade phase angle is another extremely important factor affecting the stability of flow-induced vibrations in turbomachinery. The inter-blade phase angle is the phase difference between adjacent blades, with which each blade in a rotor executes the same motion. This angle is usually determined by periodicity in turbomachinery flow caused by, for example, blade row interactions. If rotors were perfectly fabricated and tuned, flutter mode shapes would be remarkably simple; all blades would vibrate with an identical modal amplitude, but with a constant phase angle $\sigma$ between adjacent blades as shown in Figure 1.2. For a rotor with $N$ blades, the possible inter-blade phase angles are given by,

$$
\sigma_{n}=2 \pi n / N ; \quad n=0,1, \ldots, N-1 .
$$

Unsteady aerodynamic forces acting on the blades change with these inter-blade phase angles. They can sometimes function so as to stabilize the vibrations of the blades but sometimes the effect is the opposite, even if the other conditions are the same. In analyzing the stability of the blade vibration, the inter-blade phase angle must be taken into account as well as the operating condition on the compressor map, i.e., a combination of the corrected mass flow and the pressure ratio in Figure 1.1. From a computational standpoint, this circumferential periodicity is extremely useful, because it allows a reduction in the aeroelastic degrees of freedom by a factor of $N$, from $p N$ to $p$ for an equivalent blade modeled with the same $p$ degrees of freedom. 


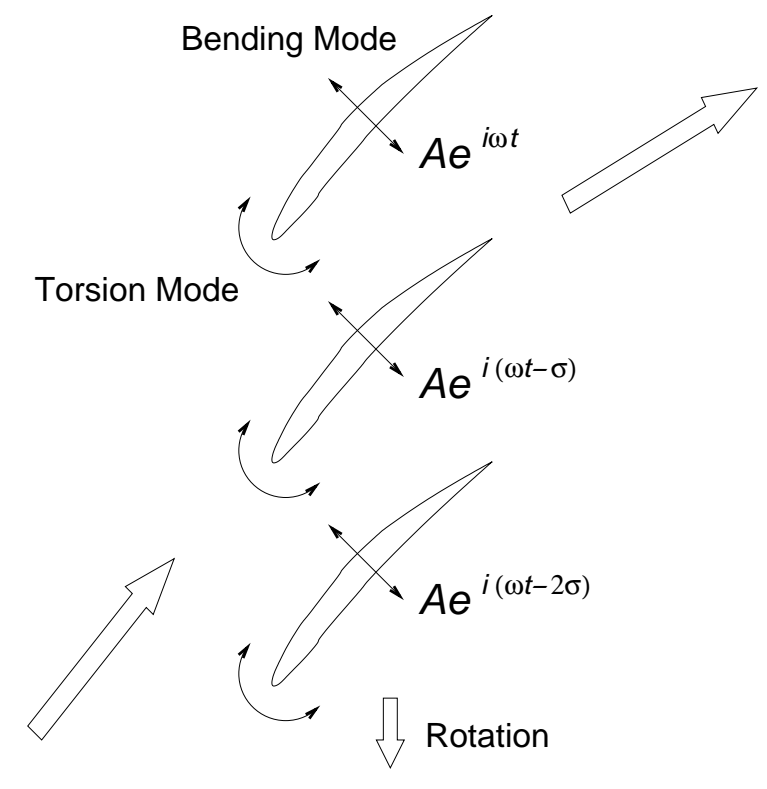

Figure 1.2: Cascade

Furthermore, turbomachinery flutter is considered to be essentially a single degreeof-freedom phenomenon. This is based on the argument that the mass ratio of a typical turbomachinery blade is so high that the effect of the aerodynamic forces on the critical modes and frequencies is negligible. The critical frequencies in air may, therefore, be identical to those in a vacuum and the frequencies of bending and torsion modes cannot coalesce, as is observed in classical bending-torsion flutter of isolated wings. Through an adjustment of modal amplitudes and inter-blade phase angles so as to extract energy from the air, flutter can occur with a pure single-degree-of-freedom mode. As discussed, adjacent blades are not necessarily in phase with each other, i.e., this strong cascade effect could bring about certain conditions where an out-of-phase aerodynamic force excites one of the blade vibration modes. These trends are, in fact, supported by some experimental evidence [6]. On the other hand, in the case of forced periodic aerodynamic excitations arising from, for example, inlet distortions or blade row interactions, the excitations are usually generated at multiples of the 
engine rotation frequency and their frequencies can coincide with a natural frequency of the blade.

\subsection{Past Efforts}

Many researchers have tried to simulate aeroelastic phenomena in turbomachinery. Their progress, in fact, is strongly associated with that of computers. In the following sections, past efforts in this field, which provided motivation for this thesis, are reviewed $[2,7,8,9]$.

\subsubsection{Linearized Unsteady Aerodynamic Theory}

It is well known that, compared to the aeroelasticity of isolated wings, the coupling between aerodynamics and structural dynamics in turbomachinery flutter problems is relatively weak. Unsteady aerodynamic analysis on turbomachinery has, therefore, generally been regarded as requiring more research attention than structural analysis. One of the methods to analyze flutter stability using only unsteady aerodynamics which has been widely used is the Energy Method [10]. It calculates the aerodynamic response of a blade row to prescribed blade motions, usually in its natural mode of vibration. Under circumstances where the coupling between the prescribed blade motion and the unsteady aerodynamic response permits the transfer of energy from the air flow to the blade, a self-excited instability occurs. In the following three sections, setting aside structural mechanics, prior work on unsteady aerodynamics around oscillating cascades is reviewed.

Because of the complexity of the flow through turbomachinery due to factors such as strong three-dimensional effects, complex shock structures, inherent unsteadiness, viscous effects especially in stall flutter, etc., a simplified analytical model has some difficulties in representing these phenomena. At the beginning of the computer 
era, researchers needed to construct simplified two-dimensional analytical models using small disturbance assumptions to linearize the governing equations. A twodimensional cascade representation of a three-dimensional multi-bladed structure is obtained by unwrapping the geometry obtained by a constant radius cut of a blade row. This means that the bending vibration of a blade may be represented by a translational motion in two-dimensions and that the torsional blade vibration becomes simply the rotation about an axis. A small disturbance formulation can be obtained with the assumption that the amplitude of vibration is small relative to the mean value. In addition, simplifications of the geometry, such as assuming small incidences and neglecting the blade thickness and camber, were necessary for the linearization. In other words, researchers were forced to analyze a flat-plate cascade operating at zero incidence to satisfy these simplifying assumptions. In order to examine the effect of the inter-blade phase angle, all blades must be assumed to vibrate with the same amplitude and with a constant phase angle between adjacent blades.

These assumptions cause the blades and their wakes to be regarded as vortex sheets. The goal of this kind of theories is to find the vorticity distribution that gives the correct velocity to satisfy the boundary conditions at the blade surfaces and in the wake. A popular model for evaluating the vorticity in subsonic incompressible flow was introduced by Whitehead [11] and extended to flat plate cascades for which the mean flow was not uniform but was actually deflected by the cascade [12]. This new model showed the importance of steady loading on the unsteady pressure distributions of vibrating cascades. Atassi and Akai $[13,14]$ also developed a two-dimensional model fully accounting for the geometry of the airfoil, though it was limited to incompressible flow. These theories suggested that unstalled pure torsional flutter is possible for low reduced frequencies, but pure bending flutter, on the other hand, should not occur in subsonic flow.

As in the case of an isolated wing, compressibility plays an important role on 
the stability of the vibration of a cascade. For turbomachinery, the first linearized potential flow analysis was performed by Lane and Friedman [15] for an oscillating flat plate staggered cascade with various inter-blade phase angles in a compressible subsonic flow. They also discovered acoustic resonance, in which a structural frequency and an acoustic frequency match for certain combinations of flow parameters, geometries and oscillation parameters. As a result, the aerodynamic damping vanishes leading to a flutter condition. Smith [16] also formulated linearized unsteady equations for compressible subsonic flat plate cascades. He conducted an experiment, and the agreement between his theory and experiment was reasonable for unloaded blade cases. Namba [17] extended the linear theory to remove the assumption of zero or very small lift and allowed cascades to have mean flow deflections. To be more realistic, mean flow analysis has been gradually replaced since then by nonuniform flow analysis.

Two-dimensional supersonic cascades also need to be studied because the relative flow becomes supersonic near the tips of low pressure compressor blades, even though the axial flow is still subsonic. For supersonic cascades, a linearized approach similar to that of the subsonic case can be used, but there are a couple of features which make the solution more difficult. One of them arises when the axial velocity is subsonic, which is of practical importance because a supersonic axial velocity very seldom occurs in real turbomachinery. Another difficulty arises with treating an infinite (countless number of blades) supersonic cascade. These difficulties were first overcome by Verdon and McCune [18] allowing them to solve the problem of infinite supersonic cascades in subsonic axial flows neglecting blade thickness, camber, and flow deflection. They examined a couple of shock reflection patterns that are determined by the Mach number and the spacing between adjacent airfoils. Their results indicated that pitching motions are unstable over a broad range of cascade 
parameter values. In their work, the solution was obtained in terms of velocity potentials, but Nagashima and Whitehead [19] formulated the unsteady equations in terms of pressure instead of velocity potentials in order to simplify the mathematical expressions and obtained almost identical results to Verdon and McCune's. Since aerodynamic loadings determine shock structures in supersonic cascades, the effects of shock structures and motions on flutter stability are more relevant than those of mean flow deflections. Bendiksen [5] examined the effects of various types of steady shock structures on flutter stability and provided an extensive explanation for the mechanism of supersonic unstalled flutter described in the previous section. Goldstein et al. [20] took quite a different approach formulating the problem in terms of velocity potentials but splitting the flow field into subsonic and supersonic regions for solving a cascade with bow shock waves detached from the leading edges of the blades. They provided for the possibility of shock induced bending flutter for relatively low reduced frequencies. They justified these results by experimentally observing bending flutter in compressors operating at high back pressures.

Following these studies, linear theories were extended to more practical methods for unsteady three-dimensional flows. Namba and Ishikawa [21] developed a lifting surface theory for three-dimensional flow in rotating subsonic, transonic and supersonic annular flat-plate cascades with fluctuating blade loadings. They showed the importance of three-dimensional effects in transonic flow, even though their theory was based on the strip hypothesis; the aerodynamics at one radial station are not coupled with the aerodynamics at any other station.

Finally, transonic flow equations are quite difficult to linearize because the governing equations are inherently nonlinear and would require linearization of the subsonic regions and supersonic flow regions independently. No relevant publications have been found in the literature regarding linearized theories for transonic flow in turbomachinery. 


\subsubsection{Time-Linearized Computations for Unsteady Aerody- namics}

One of the lessons learned from the application of linearized theories is that effects due to the steady blade loading have to be included to model the flow in a cascade accurately. The presence of strong shocks in the flow as well as the camber and thickness of the blade need to be accounted for, especially for transonic flows. Because of these modeling difficulties, a more realistic approach called time-linearization, in which the unsteady flow is regarded as a small perturbation of the fully nonuniform, possibly nonlinear, compressible steady flow, has been proposed. Using the timelinearized approach, the governing equations are linearized about a nonlinear steady or mean operating condition. The unsteady small disturbance quantities are assumed to be harmonic in time with frequency $\omega$, i.e., unsteady quantities are proportional to $e^{j \omega t}$, so that the time derivative $\partial / \partial t$ is represented by $j \omega$, where $j=\sqrt{-1}$. The resulting equations in terms of linear variable coefficients are discretized on a computational grid using conventional finite difference, finite volume, or finite element

approaches and solved numerically. Verdon and Casper [22] solved for the unsteady flow about a steady mean flow determined by the full potential equations with embedded supersonic regions and shocks on a subsonic flow field. They solved the linearized unsteady equations using a finite difference approximation and an implicit time integration. Whitehead [23] used a finite element approximation to solve the full potential flow equation on triangular elements generated around a cascade. Although he captured shocks rather than fitted them in his method, he still did not account for the production of steady and unsteady entropy and vorticity across shocks because of the assumption of potential flow. To avoid these errors, Hall and Crawley [24] introduced a time-linearized Euler analysis, in which the Euler equations are linearized 
about their steady solution. The resulting linearized unsteady equations are formulated in terms of the perturbation amplitudes. They used finite volume schemes for both steady and unsteady calculations. Hall and Lorence [25] afterwards extended their analysis to three-dimensions using a multigrid acceleration technique in their implementation.

All of the previous time-linearized methods assume that the time-averaged flow over a perturbation period must be the same as the steady flow, and consequently the nonlinear interaction between the unsteady flow and the time-averaged flow is completely neglected. Recent results, however, suggest that there are cases where the steady flow does not represent the actual mean flow. To eliminate this assumption, Ning and He [26] developed a method in which a time-averaged flow is used as the basis for harmonic perturbations. Their quasi-three-dimensional results for an oscillating cascade showed that their method can considerably improve the results compared to methods with a steady mean flow when the nonlinearity is strong, such as in transonic flows.

\subsubsection{Time-Marching Computations for Unsteady Aerody- namics}

Because their computation is too expensive, nonlinear unsteady aerodynamic theories are still not available for practical use. There are, however, situations where even small amplitude blade motions can lead to very large amplitude shock motions. In order to represent such shock motions, it is necessary to use at least the Euler equations, which can obtain the correct shock jump conditions, shock locations and velocities, and shock motion amplitudes and phase lags. Furthermore, the Reynolds-averaged Navier-Stokes (RANS) equations can account for potentially important unsteady flow 
phenomena, e.g., phenomena associated with boundary layer displacement, separation, etc. In order to capture such detailed unsteady behavior, these equations must be integrated in time to provide the solution at each period with deforming grids used to simulate the prescribed blade motions. In this section, the development of nonlinear unsteady aerodynamic theories are reviewed starting with the Euler equations, and then moving to the RANS equations.

The first time-marching method to solve the unsteady perturbation equations on vibrating two-dimensional flat plate cascades in compressible flow was developed by $\mathrm{Ni}$ and Sisto [27]. Their method can actually be considered as a hybrid of the time-marching and time-linearized methods since the equations are solved in a timemarching manner after being developed based on the time-linearized Euler equations. After their work, a method for solving the fully time-marching two-dimensional Euler equations was not reported until Fransson [28] applied these equations to vibrating cascades with thin blades. He reduced the computational region to one blade passage with the implementation of periodic boundary conditions. Taking advantage of the nonlinearity of the Euler equations, Gerolymos [29] focused on the shock motions in transonic flow for a cascade with supersonic inflow and a subsonic axial velocity component. He developed an algorithm to integrate the Euler equations using the MacCormack scheme with a finite difference formulation. L. He [30] took a different approach in solving the quasi-three-dimensional Euler equations, based on a cell-vertex finite volume discretization in space and the Runge-Kutta integration in time. He used a zonal moving mesh technique, in which only subregions near oscillating blades were moved, and the Direct Store method [31] for phase-shifted periodic boundary conditions.

It is not surprising that these methods are gradually being extended to threedimensions. Gerolymos [32] presented an algorithm for the three-dimensional unsteady Euler equations. The equations were discretized in finite volume formulations 
and integrated in time using the Runge-Kutta scheme. Similarly, Peitsch et al. [33] developed a three-dimensional Euler code adding a convergence acceleration method called the Foothold technique in which values on the periodic boundaries can be approximated over a period using interpolation.

In order to understand and predict the importance of viscous effects on the unsteady flows associated with blade vibrations, time-accurate Navier-Stokes analysis is necessary. Since the direct simulation of turbulence still lies far beyond the computer capabilities, either the thin-layer Navier-Stokes (TLNS) equations or the RANS equations need to be solved to obtain a viscous solution. The TLNS equations are solved by neglecting the viscous terms in the direction along the body. Both the TLNS and RANS equations are solved together with an appropriate turbulence model [34]. Since Huff [35] accomplished the first unsteady RANS simulation of two-dimensional vibrating cascaded airfoils, many researchers have been working on the development of unsteady Navier-Stokes codes. Siden [36] developed such a code for simulating quasi-three-dimensional unsteady viscous compressible flows. He accounted for viscosity by including a two-layer algebraic turbulence model. L. He and Denton [37, 38] developed a unique approach in which solutions of the Euler equations and integral boundary layer equations were coupled to provide unsteady viscous flow solutions. They showed that the inclusion of viscous effects changed the pattern of unsteady shock wave motions because the viscous blockages in the flow passage significantly affected both steady and unsteady flow fields. Faster convergence has been pursued since both TLNS and RANS computations still require high computational cost. Ji and Liu [39] took advantage of the message passing interface (MPI) to reduce computational wall clock time using multiple CPUs. They compared the Direct Store method on a single passage domain and MPI on multiple passage domains using a quasi-three-dimensional RANS solver, and showed that MPI reached the final periodic solution significantly faster than the single passage computation. They also 
implemented the multigrid method and the dual time stepping scheme discussed in Chapter. 3.

Finally, in order to analyze the aeroelastic stability of a real engine, threedimensional Navier-Stokes analysis is the most practical of aerodynamic tools for sim-

ulating the viscous flow around vibrating cascades with complete geometries. There are currently several three-dimensional Navier-Stokes codes available for such simulations. L. He and Denton [40] extended their previous work to a three-dimensional time-marching method for solving the TLNS equations. They used the cell-vertex finite volume scheme in space and the four-stage Runge-Kutta scheme in time and provided viscous flow solutions around the oscillating NASA Rotor 67 [41] transonic fan rotor. Bakhle et al. [42] developed an aeroelastic code called TURBO-AE, in which a finite volume discretization in space, the Gauss-Seidel iteration for the integration in time, and the Baldwin-Lomax turbulence model [43] were applied.

\subsubsection{Fluid/Structure Coupled Computations}

As discussed in Section 1.2.1, Carta's Energy Method [10] assumes that flutter occurs in one of the natural modes of the structure, which leads to the prescription of blade motions in computations and the simplification of the expression for the work per cycle. An experimental measurement of a fluttering fan [44], however, demonstrates instead that this assumption does not hold for the tip region of a low aspect ratio wide chord fan. Experiments show that the phase angle between bending and torsion may be changing and the blade motion may not be consistent as flutter is approached. This trend implies the necessity of a full fluid/structure coupled computation.

As long as both the structural model and the fluid equations are linear, the aeroelastic equations of motion can be solved with linearized unsteady aerodynamic coefficients to obtain amplitudes of displacements for an arbitrary frequency. For single-degree of freedom problems, Whitehead [45] solved the equations of motion 
in terms of the angular displacement of the blade with aerodynamic moment coefficients which were calculated by his own linear aerodynamic theory [11] for unsteady two-dimensional incompressible flows. Bendiksen and Friedmann [46] examined the possibility of bending/torsion coupled flutter by developing a method for determining the aeroelastic stability of a cascade. They coupled Whitehead's linear solution for incompressible unsteady flow [12] and the Typical Section structural model [47] with bending and torsional degrees of freedom. Their results illustrate that the bending/torsion interaction has a pronounced effect on the flutter boundary. To be more realistic regarding the structural model, Kaza and Kielb [48] used a straight, slender, twisted and nonuniform elastic beam with symmetric cross sections to represent a blade and Smith's linear theory [16] as a flow solver, and presented flutter boundaries for a mistuned blade row.

It is generally reasonable to assume that the structural problem remains fairly linear but that the aerodynamic problem generally does not, and the combined problem is to some extent nonlinear, especially in transonic flow. The coupling of a linear structural model and a fully nonlinear aerodynamic model requires a time-marching method and determines the frequency of the problem rather than specifying it as an input parameter. Gerolymos's method [49] is in a sense a time-marching method but the coupling relations are still formulated in the frequency domain, even though he uses his own three-dimensional Euler code [32]. He computed the initial vibratory modes using a finite element analysis, then updated the frequencies and the mode shapes with unsteady Euler solutions of the previous period to satisfy the structural equation at the end of the period, and repeated this procedure using the updated vibratory modes for the next period until the solution converged.

While Gerolymos's method might not be computationally expensive, it cannot demonstrate how the vibration decays or diverges in a time history. A full timemarching method is useful in that sense, because the aerodynamic and structural 
equations are integrated simultaneously or alternately in time to calculate the aeroelastic response which can be expressed in a time history. Reddy et al. [50] presented a full time-marching method with a two-dimensional unsteady aerodynamic Euler solver and the Typical Section structural model for each blade of the cascade. L. He [51] focused on the mechanism of rotating stall and stall flutter in turbomachinery and developed a two-dimensional coupled method. He solved the aeroelastic system for multiple blade passages in multiple blade rows by integrating the unsteady NavierStokes equations and the Typical Section structural equations simultaneously in time using the Runge-Kutta scheme. The most noticeable work on the fluid/structure coupled computations is done by Vahdati, Imregun and their colleagues. They performed dynamic aeroelastic computations of NASA's Rotor 67 [52] and those of a wide chord fan blade to predict flutter boundaries at 75 to 85 percent rotation speed [53] using unstructured meshes, a finite element RANS solver and a finite element linear structural model. In their work, identical structure and fluid surface meshes are used to avoid interpolations between the fluid and the structure. This particular type of flutter with these rotation speeds corresponds to supersonic stall flutter, but the results indicate that it can occur without stall. Their approach does not model the tip clearance, and the relative rotation of the annulus casing wall is not accounted for. Gottfried and Fleeter [54] followed their work by also making use of a finite element model which can handle both the structural equations and the three-dimensional Euler equations.

\subsection{Motivations of the Research}

The literature reviewed in the previous three sections is only a brief synopsis of the overall research in the area of turbomachinery aeroelasticity. Many computational 
studies have been carried out to predict aeroelastic stability in turbomachinery. However, the efforts mostly concentrate on recreating the phenomena and understanding their physical mechanisms; thus they often assume two-dimensional prescribed blade motions and therefore are not practical tools for designing high performance turbomachinery components. The same trend can be seen in experiments due to the difficulty of performing experiments on such phenomena and carrying out measurements. Similarly to the computational studies, in fact, all recent experimental studies on cascades have been directed at studying the unsteady flow through two-dimensional oscillating cascades, and not at predicting actual flutter boundaries. There have only been a few efforts to carry out flutter tests on rotating fans [6,44], but they unfortunately have not provided enough information, especially for the geometry and the structural properties of the blades, to compare properly with computational results. It can be said from these trends that, since past computational and experimental studies have revealed the mechanisms of the aeroelastic instability, studies should be more directed towards developing a practical computational tool for predicting the aeroelastic stability in actual turbomachinery geometries that can be validated concurrently by experiment.

As a matter of fact, recent progress in computer processing speeds and computational methods are revolutionizing the design of aircraft and turbomachinery. For some kinds of structural and aerodynamic designs, experiments have been mostly replaced by computational simulations. As far as aeroelastic predictions are concerned, they are, for the most part, still based on the classical linearized unsteady aerodynamic analysis. Since the experimental validation of the aeroelastic performance of aircraft and turbomachinery is greatly concerned with operational safety, designers want to predict the aeroelastic stability as accurately as possible before they fabricate the aircraft or turbomachinery component. Therefore, computational aeroelastic simulations would be the most logical alternative to the experimental validation of the 
design. To achieve this, it is necessary to integrate a nonlinear unsteady Navier-Stokes flow solver and a practical structural solver for turbomachinery. One possibility for this integration is to share the grid points on the interface and solve both equations with the same numerical method to carry out solutions simultaneously as Vahdati and Imregun $[52,53]$ did. On the other hand, since the methodologies of each individual discipline have matured independently, each solver has evolved to use a different type of grid generation, a different discretization method and a different time integration scheme so that high accuracy and efficiency can be individually achieved. In order to take advantage of the maturity of both types of solver, a more reasonable alternative would be to construct an interface procedure between a flow solver and a structural solver, in which the two solvers exchange the interface information and update the fluid and structural variables alternatively.

The present study [55] explores this possibility by integrating an unsteady NavierStokes flow solver and a finite element structural solver for aeroelastic problems in turbomachinery, using advanced fluid/structure coupling techniques: load transfer, deformation tracking, and synchronization. The flow solver used here is an unsteady three-dimensional Navier-Stokes solver called $\operatorname{TFLO}[56,57,58,59]$, originally developed to simulate unsteady flows due to blade row interactions in turbomachinery. The structural solver used here is one of the accepted industrial standards, $M S C / N A S T R A N$. The integrated aeroelastic solver in this study is validated by comparing solutions of some standard configurations with other computational and experimental works, and it is then extended to predict the flutter boundary of the fan stage of the NASA Rotor 67 geometry. 


\section{Chapter 2}

\section{Governing Equations}

An aeroelastic problem can be divided into two different components: aerodynamics and structural mechanics. They are individually governed by their own basic principles, and they usually have different frames of reference when their governing equations are formulated: while the fluid equations are typically written using spatial coordinates as independent variables (Eulerian frame), the structural equations are usually formulated using material coordinates (Lagrangian frame). Regardless of the frame used, as long as a structure exists within a fluid, the two components interact through structural deformations, aerodynamic pressure and viscous forces, and heat transfer effects. In this study, aeroelastically small effects such as viscous forces and heat conduction are neglected. However, there still are some basic principles that represent the physics for transforming deformations and pressures between the fluid and the structure which use different frames of reference. This chapter explains the individual governing equations for both unsteady aerodynamics and structural mechanics, and the principles that must be conserved for fluid/structure interactions through structural deformations and aerodynamic pressures. 


\subsection{Unsteady Aerodynamics}

For a fluid in motion, the velocity vector may be different at each location within the fluid. In order to describe the physical properties of a moving fluid, it is convenient to use an Eulerian frame of reference, in which the observer focuses attention on a particular volume in space and studies the fluid as it passes through the volume. This particular volume is called a control volume. A spatial coordinate system, which is fixed in space, is used to describe control volumes and flow variables, such as density, velocity, pressure, temperature, etc. Every flow variable is regarded as a function of the spatial coordinates and time for an unsteady flow. Thus, an arbitrary scalar quantity $\chi$ can be expressed as $\chi\left(x_{1}, x_{2}, x_{3}, t\right)$. This expression can hold for any kind of unsteady flow, but there is a difference between the formulation for an unsteady flow with a fixed control volume in space and that for a moving control volume which is necessary in the computation of a dynamic fluid/structure system which has moving boundaries that displace the computational mesh. In the following sections, fundamental physical principles including conservation of mass, momentum and energy, are applied to a finite moving control volume. The equations so obtained are expressed in integral form and are called the conservation form of the governing equations. In integral form these equations can be conveniently discretized using a finite volume scheme discussed in Chapter 3. In addition, because the derivation of the governing equations can be most easily carried out in a Cartesian system of coordinates, it is employed hereafter.

As far as the behavior of a fluid is concerned, the material medium of the fluid is modeled in an approximate sense, by using a set of equations describing its mechanical and thermodynamic properties. These properties are called constitutive relations. In this study, the behavior of a fluid follows some constitutive relations including the concept of a Newtonian fluid the viscous stress-strain relationship, the Fourier law of 
heat conduction, and the perfect gas law.

\subsubsection{Reynolds' Transport Theorem}

In order to derive the governing equations of fluid mechanics, it is convenient to make use of the Reynolds' transport theorem. Consider the time-rate of change of a scalar quantity $\chi$ within a control volume $V(t)$, with outward unit normal vector $\boldsymbol{n}$ and local boundary velocity $\boldsymbol{b}$ varying over the surface $S(t)$ of the control volume. Then the theorem can be expressed as,

$$
\frac{d}{d t} \int_{V(t)} \chi d V=\int_{V(t)} \frac{\partial \chi}{\partial t} d V+\int_{S(t)} \chi(\boldsymbol{b} \cdot \boldsymbol{n}) d S .
$$

Equation 2.1 states that the time-rate of change of the total amount of a scalar quantity $\chi$ in a varying volume $V(t)$, enclosed by the surface $S(t)$ is composed of two terms: the time-rate of change of the scalar integrated over the entire volume and the effect of the changing size of the volume on the total amount of scalar in the volume. The theorem is often applied in a Lagrangian frame, in which case the boundary velocity $\boldsymbol{b}$ is just the fluid velocity $\boldsymbol{u}=\left(u_{1}, u_{2}, u_{3}\right)$ and the statement becomes,

$$
\frac{d}{d t} \int_{V(t)} \chi d V=\int_{V(t)} \frac{\partial \chi}{\partial t} d V+\int_{S(t)} \chi(\boldsymbol{u} \cdot \boldsymbol{n}) d S
$$

\subsubsection{Conservation of Mass}

In developing the governing equations, it is sometimes useful to associate them with the Lagrangian frame where a finite control volume $V$ is moving with the fluid in question. In addition, if the closed surface $S$ is attached to an individual fluid particle

and consequently both $S$ and $V$ have the same velocity as the particle, the total mass of the control volume must be constant. In other words, its time rate of change must be zero. Therefore, 


$$
\frac{d}{d t} \int_{V} \rho d V=0
$$

Since the control volume has the same velocity $\boldsymbol{u}$ as the fluid particle, applying the Reynolds' transport theorem,

$$
\int_{V} \frac{d \rho}{d t} d V+\int_{S} \rho(\boldsymbol{u} \cdot \boldsymbol{n}) d S=0 .
$$

On the other hand, when the Eulerian frame is applied to the same flow field, the Reynolds' transport theorem still can be valid for a control volume with moving boundary whose velocity is $\boldsymbol{b}$. Then,

$$
\frac{d}{d t} \int_{V(t)} \rho d V=\int_{V(t)} \frac{d \rho}{d t} d V+\int_{S(t)} \rho(\boldsymbol{b} \cdot \boldsymbol{n}) d S .
$$

Notice that the most important contribution to $\boldsymbol{b}$ in turbomachinery is due to the rotation of the wheel. For a rigid grid fixed to the casing or a stator, $\boldsymbol{b}=0$. For a rigid grid fixed to a rotor without elastic deformations, $\boldsymbol{b}=\vec{\Omega} \times \boldsymbol{r}$, where $\Omega$ is the angular velocity of rotation defined in the $x_{1}$-direction, and $\boldsymbol{r}$ is the displacement vector from the rotor axis. In aeroelastic calculations, surface velocities due to the aeroelastic deformations are added to $\boldsymbol{b}$.

Equating the first right-hand term in 2.5 to the equivalent term in 2.4 gives,

$$
\frac{d}{d t} \int_{V(t)} \rho d V+\int_{S(t)} \rho(\boldsymbol{u}-\boldsymbol{b}) \cdot \boldsymbol{n} d S=0 .
$$

This resulting equation states the conservation of mass for an unsteady flow with moving boundaries.

\subsubsection{Conservation of Momentum}

Conservation of momentum, sometimes referred to as Newton's second law, says that the net force on the fluid element equals its mass times the acceleration of the element. 
The net forces on the fluid particles in the volume $V(t)$ along the three axes can be expressed as the collection of the surface stress vector $\boldsymbol{T}$ acting on $S(t)$ and the body force vector $\boldsymbol{G}$ acting throughout $V(t)$. The expression for this principle can be written in a similar form, using Reynolds' transport theorem as in the case of conservation of mass,

$$
\frac{d}{d t} \int_{V(t)} \rho \boldsymbol{u} d V+\int_{S(t)} \rho \boldsymbol{u}(\boldsymbol{u}-\boldsymbol{b}) \cdot \boldsymbol{n} d S=\int_{S(t)} \boldsymbol{T} d S+\int_{V(t)} \rho \boldsymbol{G} d V .
$$

Here the body force vector $\boldsymbol{G}$, defined as an overall force proportional to the amount of mass, may represent gravity or an electro-magnetic effect. The surface stress vector $\boldsymbol{T}$ can be expressed in terms of the stress tensor $\sigma_{i j}$ as follows;

$$
\boldsymbol{T}=\sigma_{i j} \boldsymbol{e}_{i}
$$

where $\boldsymbol{e}_{i}$ is the unit vector in $i$-direction of the Cartesian coordinate. The surface stress tensor can be decomposed into the viscous stress tensor $\tau_{i j}$ and the hydrostatic pressure $p$. The first constitutive relation is introduced here. A Newtonian fluid is defined to be one for which the viscous stress contributes only to the deformation of the fluid, but not to the translation or rigid body rotation. On making use of this definition, the number of constants in the constitutive relation remarkably reduces to just one, when use of the Stokes hypothesis is made. The Stokes hypothesis postulates that the hydrostatic pressure can be chosen to be equal to the mean of the normal stresses, $\sigma_{i i}=-3 p$, that the bulk viscosity is assumed to be negligible, $\tau_{k k}=0$, and the viscous stress tensor is symmetric, $\tau_{i j}=\tau_{j i}$. Finally the expression for the surface stress tensor for a Newtonian fluid is given as follows;

$$
\sigma_{i j}=-p \delta_{i j}+\tau_{i j}=-p \delta_{i j}+\mu\left[\frac{\partial u_{i}}{\partial x_{j}}+\frac{\partial u_{j}}{\partial x_{i}}\right]-\frac{2}{3} \mu\left[\frac{\partial u_{k}}{\partial x_{k}}\right] \delta_{i j},
$$

where $\mu$ is the coefficient of viscosity and $\delta_{i j}$ is the Kronecker's delta. 


\subsubsection{Conservation of Energy}

The physical principle of conservation of energy is nothing more than the first law of thermodynamics. The effects that can change the energy stored in $V(t)$, i.e., the internal energy $e$ and the kinetic energy $\frac{u^{2}}{2}=\frac{1}{2}\left(u_{1}^{2}+u_{2}^{2}+u_{3}^{2}\right)$ of the fluid, can be divided into two categories. One is the rate of work done on the fluid by external forces, and the other is the net flux of heat into the fluid. The rate of work done consists of that done by the surface stress vector $\boldsymbol{T}$ on the boundary $S(t)$ and that done by the body force $\boldsymbol{G}$ throughout the volume. The net flux of heat consists of the rate of conductive heat loss $\boldsymbol{-} \boldsymbol{q}$ through the surface and the rate of volumetric energy addition $Q$, which can represent radiation, chemical heat release, or resistive losses produced by an electric current, to name a few. Based on the Reynolds transport theorem, the conservation of energy statement can be written by collecting all of the above terms as follows,

$$
\begin{array}{r}
\frac{d}{d t} \int_{V(t)} \rho\left(e+\frac{\boldsymbol{u}^{2}}{2}\right) d V+\int_{S(t)} \rho\left(e+\frac{\boldsymbol{u}^{2}}{2}\right)(\boldsymbol{u}-\boldsymbol{b}) \cdot \boldsymbol{n} d S \\
=\int_{S(t)} \boldsymbol{T} \cdot(\boldsymbol{u}-\boldsymbol{b}) d S+\int_{V(t)} \rho \boldsymbol{G} \cdot \boldsymbol{u} d V-\int_{S(t)} \boldsymbol{q} \cdot \boldsymbol{n} d S+\int_{V(t)} Q d V .
\end{array}
$$

The heat conducting behavior of an isentropic fluid under ordinary conditions of pressure and temperature is represented quite well by a linear relation between the temperature gradient and the heat flux vector $\boldsymbol{q}$. This is one of the constitutive relations called the Fourier law of heat conduction which is described by

$$
\boldsymbol{q}=-k \nabla T
$$

where $T$ is the temperature of the fluid, and $k$ is the coefficient of thermal conductivity, which is assumed to depend on temperature alone and does not depend on density in 
this study. First, the temperature dependence of the viscosity coefficient is modeled by Sutherland's law

$$
\frac{\mu}{\mu_{0}}=\left(\frac{T}{T_{0}}\right) \frac{T+110 K}{T+T_{0}},
$$

where the subscript 0 denotes the condition at a reference state, which is usually taken to be a freestream condition. The heat conduction coefficient is then determined by assuming a constant Prandtl number, $P r$,

$$
\operatorname{Pr}=\frac{c_{p} \mu}{k},
$$

where $c_{p}$ is the specific heat at constant pressure.

In order to study the compressible flow of gas in equilibrium, thermodynamic equilibrium conditions must be paid attention to in order to obtain another constitutive relation for describing the physical properties of the fluid. In this study, the fluid of interest is defined to be a thermally perfect gas defined by

$$
p=\rho R T,
$$

where $R$ is the specific gas constant. This equation is sometimes labeled the thermal equation of state. The other property used here is the fact that the gas is assumed to be calorically perfect as defined by

$$
e=c_{v} T,
$$

where $c_{v}$ is the specific heat at constant volume. This equation is sometimes labeled the caloric equation of state. 


\subsubsection{The Reynolds-Averaged Navier Stokes Equations}

The collection of these three conservation laws, Equations 2.6, 2.7 and 2.10, and the constitutive relations, Equations 2.9, 2.11, 2.14 and 2.15, are called the Navier-Stokes equations, which are in general considered to be capable of describing Newtonian turbulent viscous flows. However, solving high Reynolds-number flows directly with the Navier-Stokes equations requires such small temporal and spatial scales associated with turbulent fluctuations that, for complex geometries, a tremendous amount of mesh points far beyond the limits of the current computer technology would be required to carry out such a computation. Usually in engineering, the mean values of the flow variables are the quantities of interest especially in predicting the performance of machinery in design. It is possible to reconstruct the Navier-Stokes equations for the mean values of the flow variables by taking a time average of the flow variables over a sufficiently long period, $\tau$, compared with the frequencies of turbulent fluctuations, and arrive at the Reynolds-averaged Navier-Stokes equations.

An unsteady turbulent flow can often be idealized as consisting of two parts: a slowly varying mean flow plus a rapidly fluctuating component related to turbulence. The more disparate these two time scale are the more realistic this description becomes. Each variable $\chi$ in the Navier-Stokes equations can be written as the sum of the mean value $\bar{\chi}$ over a time interval $\tau$ and a time-dependent fluctuation $\chi^{\prime}$,

$$
\chi=\bar{\chi}+\chi^{\prime}=\frac{1}{\tau} \int_{t}^{t+\tau} \chi d t+\chi^{\prime} .
$$

This averaging is used for density, pressure, and both the stress tensor and the heat flux.

$$
\begin{aligned}
& \rho=\bar{\rho}+\rho^{\prime}, \quad p=\bar{p}+p^{\prime}, \\
& \tau_{i j}=\overline{\tau_{i j}}+\tau_{i j}^{\prime}, \quad \boldsymbol{q}=\overline{\boldsymbol{q}}+\boldsymbol{q}^{\prime} .
\end{aligned}
$$

For compressible flow, Rubesin and Rose [60] proposed the concept of Farve averaging 
in which a conservative variable $\chi$ is averaged in terms of its mass-weighted value to form an alternative form of the sum of the mean value $\tilde{\chi}$ and the time-dependent fluctuation $\chi^{\prime \prime}$,

$$
\chi=\tilde{\chi}+\chi^{\prime \prime}=\frac{\int_{t}^{t+\tau} \rho \chi d t}{\tau \bar{\rho}}+\chi^{\prime \prime} .
$$

This averaging is used for velocity, specific energy, viscosity and heat conductivity to result in a remarkable simplification of the equations hereafter. $\tau$ is assumed to be sufficiently large to ensure that the mean of the fluctuations $\chi^{\prime}$ and $\chi^{\prime \prime}$ are zero.

$$
\begin{aligned}
& \boldsymbol{u}=\tilde{\boldsymbol{u}}+\boldsymbol{u}^{\prime \prime}, \\
& E=e+\frac{\boldsymbol{u}^{2}}{2}=\tilde{E}+E^{\prime \prime}
\end{aligned} \quad e=\tilde{e}+e^{\prime \prime},
$$

Consider a flow in which body forces and volumetric heat addition are not present, i.e., $\boldsymbol{G}=0$ and $Q=0$, for a domain $\Omega$ with boundary $\partial \Omega$. Substituting the appropriate decomposition of the flow variables in the Navier-Stokes equations and taking a time average of the equations yields the following equations after some algebra and simplifications,

$$
\begin{gathered}
\frac{d}{d t} \int_{V(t)} \bar{\rho} d V+\int_{S(t)} \bar{\rho}(\tilde{\boldsymbol{u}}-\boldsymbol{b}) \cdot \boldsymbol{n} d S=0, \\
\frac{d}{d t} \int_{V(t)} \bar{\rho} \tilde{\boldsymbol{u}} d V+\int_{S(t)}\left\{\bar{\rho} \tilde{\boldsymbol{u}} \cdot(\tilde{\boldsymbol{u}}-\dot{\boldsymbol{x}})+\bar{p} \delta_{i j}\right\} \boldsymbol{n} d S=\int_{S(t)}\left(\bar{\tau}_{i j}-\overline{\rho u_{i}^{\prime \prime} u_{j}^{\prime \prime}}\right) \boldsymbol{n} d S, \\
\frac{d}{d t} \int_{V(t)} \bar{\rho} \tilde{E} d V+\int_{S(t)}(\bar{\rho} \tilde{E}+\bar{p})(\tilde{\boldsymbol{u}}-\boldsymbol{b}) \cdot \boldsymbol{n} d S= \\
\int_{S(t)}\left\{\left(\bar{\tau}_{i j}-\overline{\rho u_{i}^{\prime \prime} u_{j}^{\prime \prime}}\right)(\tilde{\boldsymbol{u}}-\boldsymbol{b})-\left(\overline{\boldsymbol{q}}-\overline{\rho e^{\prime \prime} \boldsymbol{u}^{\prime \prime}}\right)+\left(\frac{1}{\left(\tau_{i j}-\frac{1}{2} \rho u_{i}^{\prime \prime} u_{j}^{\prime \prime}\right) \boldsymbol{u}^{\prime \prime}}\right\} \cdot \boldsymbol{n} d S .\right.
\end{gathered}
$$


The mean energy dissipation $\overline{\left(\tau_{i j}-\frac{1}{2} \rho u_{i}^{\prime \prime} u_{j}^{\prime \prime}\right) \boldsymbol{u}^{\prime \prime}}$ is neglected following an order of magnitude estimate. The above equations are equivalent to their laminar counterparts except for two terms: the Reynolds stress $-\overline{\rho u_{i}^{\prime \prime} u_{j}^{\prime \prime}}$ and the Reynolds heat flux $-\overline{\rho e^{\prime \prime} \boldsymbol{u}^{\prime \prime}}$. They can be written in exactly the same form as the Navier-Stokes equations with the following redefinition of the viscous stresses and heat fluxes,

$$
\begin{aligned}
\tau_{i j, t o t a l} & =\bar{\tau}_{i j}-\overline{\rho u_{i}^{\prime \prime} u_{j}^{\prime \prime}}, \\
q_{j, t o t a l} & =\bar{q}_{j}-\overline{\rho e^{\prime \prime} u_{j}^{\prime \prime}} .
\end{aligned}
$$

While the mean value of the viscous stress and heat conduction are related to the mean flow variable with constitutive relations represented by the Stokes hypothesis, the Fourier law and Sutherland's law, there is not a known constitutive relation to associate the Reynolds stress and the Reynolds heat flux with the mean flow variables. This requires the introduction of turbulence closure models.

\subsubsection{Conservation Law Form}

The conservation form of the governing equations is convenient for use with numerical approaches because the conservations of mass, momentum, and energy in the conservation form can all be expressed by the same generic equation. After elimination of the bars and tildes in the Equations 2.1.2 and proper nondimensionalization, the conservation laws for the appropriate averaged variables can be written in an integral form of the same generic equation as

$$
\frac{d}{d t} \int_{\Omega} \boldsymbol{W} d V+\int_{\partial \Omega} \boldsymbol{F} \cdot d \boldsymbol{S}=\frac{1}{R e} \int_{\partial \Omega} \boldsymbol{f} \cdot d \boldsymbol{S}
$$

where $\boldsymbol{S}=\left(S_{1}, S_{2}, S_{3}\right)$ are the components of the projected areas in the three Cartesian coordinate directions, $\boldsymbol{W}$ represents the conserved quantity, $\boldsymbol{F}=\left(\boldsymbol{F}_{1}, \boldsymbol{F}_{2}, \boldsymbol{F}_{3}\right)$ 
are the corresponding flux vectors

$$
\boldsymbol{W}=\left(\begin{array}{c}
\rho \\
\rho u_{1} \\
\rho u_{2} \\
\rho u_{3} \\
\rho E
\end{array}\right), \quad \boldsymbol{F}_{i}=\left(\begin{array}{c}
\rho\left(u_{i}-b_{i}\right) \\
\rho u_{1}\left(u_{i}-b_{i}\right)+p \delta_{1 i} \\
\rho u_{2}\left(u_{i}-b_{i}\right)+p \delta_{2 i} \\
\rho u_{3}\left(u_{i}-b_{i}\right)+p \delta_{3 i} \\
\rho H\left(u_{i}-b_{i}\right)
\end{array}\right)
$$

and $\boldsymbol{f}=\left(\boldsymbol{f}_{1}, \boldsymbol{f}_{2}, \boldsymbol{f}_{3}\right)$ is the vector of viscous source terms

$$
\boldsymbol{f}_{i}=\left(\begin{array}{c}
0 \\
\tau_{1 i} \\
\tau_{2 i} \\
\tau_{3 i} \\
\tau_{i j} u_{j}+q_{i}
\end{array}\right)
$$

The thermodynamic static pressure $p$ can be related to the total energy $E$ as follows

$$
p=(\gamma-1) \rho\left(E-\frac{\boldsymbol{u}^{2}}{2}\right),
$$

where $\gamma$ is the ratio of specific heats. In addition,

$$
H=E+\frac{p}{\rho}=\frac{c^{2}}{\gamma-1}+\frac{\boldsymbol{u}^{2}}{2}
$$

the total enthalpy, is important in fluid dynamics because it is found to be a constant in many important flows. $c$ is the isentropic speed of sound defined by

$$
c^{2}=\frac{\gamma p}{\rho}
$$

In order to maintain the equations in the same form independently of the use of units, all the variables in Equations 2.23 are all properly nondimensionalized with the major reference values. They are listed below, 


$$
\begin{array}{ll}
\text { length: } & l_{\text {ref }}=\text { Chord of airfoil or blade } \\
\text { pressure: } & p_{\text {ref }}=p_{\infty} \\
\text { density: } & \rho_{\text {ref }}=\rho_{\infty} \\
\text { velocity: } & V_{\text {ref }}=\sqrt{p_{r e f} / \rho_{r e f}} \\
\text { energy: } & E_{r e f}=V_{r e f}^{2} \\
\text { viscosity: } & \mu_{\text {ref }}=\mu_{\infty} \\
\text { heat conductivity: } & k_{\text {ref }}=\mu_{\infty} / c_{p}
\end{array}
$$

$R e$ is the Reynolds number based on the above reference values,

$$
R e=\frac{\rho_{r e f} V_{r e f} l_{r e f}}{\mu_{r e f}} .
$$

Notice that when $R e$ is large, the right hand side of Equations 2.23 becomes relatively small enough to ignore it in most of the flow field except near solid walls. In other words, most of the flow field can be properly represented by the Euler equations and only the regions near the wall experience a strong effect of viscosity. Since the viscous terms in Equations 2.23 cause rapid variations in the solution, the regions with strong effect of viscosity requires fine mesh sizes for stability.

\subsection{Structural Mechanics}

In order to determine the distribution of static or dynamic displacements in a structure evolving under a set of prescribed external force and temperature loadings, the basic equations of the theory of elasticity must be formulated and solved. For a general three-dimensional structure these governing laws are composed of fifteen equations for fifteen unknown variables, which are three displacements, six strains, and six stresses. The governing equations are then three equations of motion, six strain-displacement equations, and six stress-strain relations. In this section, all the basic equations of 
the elasticity are summarized, and the principle of virtual work, on which the finite element method is based, is presented to provide numerical solutions for these equations.

\subsubsection{Differential Equations of Elasticity}

Consider a material point of an elastic structure at a position $\boldsymbol{x}\left(x_{1}, x_{2}, x_{3}\right)$ without deformation. The deformed shape under a given system of loads and temperature distributions can be expressed in terms of the displacement vector $\boldsymbol{u}\left(u_{1}, u_{2}, u_{3}\right)$. In general, the deformed configuration is uniquely defined if the displacement components are given as a function of $\boldsymbol{x}$ everywhere within the structure of interest. Thus, three normal strain components $\epsilon_{i i}$ and six shear strain components $\epsilon_{i j}$ can be defined to describe the general deformation in a three-dimensional structure. For small deformations, the strain-displacement relations are linear, and the strain components are expressed as partial derivatives of the displacement $\boldsymbol{u}$,

$$
\epsilon_{i i}=\frac{\partial u_{i}}{\partial x_{i}}, \quad \epsilon_{i j}=\epsilon_{j i}=\frac{\partial u_{j}}{\partial x_{i}}+\frac{\partial u_{i}}{\partial x_{j}}
$$

Next, although the determination of the thermal stress plays an important part in the design of structures operating at high temperatures, the stress-strain equations do not include the effect of temperature in this study, because it is negligible compared to the effect of the pressure distribution imposed by the fluid. Stress is defined as a force per unit area and has three components of normal stress $\sigma_{i i}$ and six components of shear stress $\sigma_{i j}$. The normal stress and the normal strain are in general linearly proportional to each other and the constant of proportionality is called the Young's moduli $E_{i}$. In the case of uniaxial stress, lateral strains are also related by the Poisson ratio $\nu_{i}$. If the material is isotropic, i.e., its mechanical properties are not direction dependent, all the Young's moduli and the Poisson ratios are identical. 
Furthermore, in the linear stress-strain relations, all stress components are given by linear superposition. Thus the stress-strain relations are,

$$
\sigma_{i i}=\frac{E}{(1+\nu)(1-2 \nu)}\left[(1-\nu) \epsilon_{i i}+\nu\left(\epsilon_{j j}+\epsilon_{k k}\right)\right], \quad \sigma_{i j}=\frac{E}{2(1+\nu)} \epsilon_{i j} .
$$

It can be shown that the shear stresses are symmetric, i.e., $\sigma_{i j}=\sigma_{j i}$ in Equation 2.31, and this can also be verified by considering the equilibrium of moments about the $x_{1}$, $x_{2}$, and $x_{3}$ axes.

Finally, equations of motion relating the nine stress components are derived by considering equilibrium of internal and external forces acting on a small moving particle with unit volume. Under dynamic loading conditions, measured by the natural frequencies of the system, inertia forces $-\rho \ddot{\boldsymbol{u}}$ need to be considered. In addition, in actually measured dynamic responses of structures, it is observed that energy is dissipated during vibration, which is usually taken into account in vibration analysis by introducing velocity dependent damping forces $-\kappa \dot{\boldsymbol{u}}$, where $\kappa$ is the coefficient of viscous damping per unit volume. Resolving inertia forces in the $i$-direction, three partial differential equations are obtained,

$$
\frac{\partial \sigma_{i j}}{\partial x_{j}}+\rho \ddot{u}_{i}+\kappa \dot{u}_{i}=G_{i} .
$$

where $G_{i}$ represents the body force in the $i$-direction. Equation 2.32 must be satisfied at all points of the structure. The stresses $\sigma_{i j}$ vary throughout the structure, and at its boundary surface they must be in the equilibrium with the external forces applied to the surface. 


\subsubsection{The Principle of Virtual Work}

The exact solution of the partial differential equations of elasticity for complex structures can be obtained in closed form only in special cases. It is, however, possible to solve these equations in an approximate way by introducing energy methods, which are restricted to small strains and displacements so that the strain-displacement relationships can be remain linear. The most popular of the many existing energy methods assumes that the forces remain constant while the displacements are varied from $\boldsymbol{u}$ to $\boldsymbol{u}+\delta \boldsymbol{u}$, where $\delta \boldsymbol{u}$ is the virtual displacement. This assumption leads to the principle of virtual work, which states that an elastic structure is in equilibrium under a given set of load distributions if the virtual work is equal to the virtual strain energy for any virtual displacement $\delta \boldsymbol{u}$ from a compatible state of deformation $\boldsymbol{u}$. Since the inertia and damping forces are proportional to the volume of the element, they contribute to the virtual work done by the body forces. As a result, the principle is given by integrating Equation 2.32 times the virtual displacement $\delta \boldsymbol{u}$ over the structure of interest,

$$
\int_{V}\left(\frac{\partial \sigma_{i j}}{\partial x_{j}}+\rho \ddot{u}_{i}+\kappa \dot{u}_{i}\right) \delta \boldsymbol{u} d V=\int_{S} p \delta \boldsymbol{u} d \boldsymbol{S},
$$

where the right hand side corresponds to the external work done by the aerodynamic pressure on the boundary surface of the structure. Introducing the strain vector $\epsilon$ and the stress vector $\boldsymbol{\sigma}$ as

$$
\boldsymbol{\epsilon}=\left(\epsilon_{11}, \epsilon_{22}, \epsilon_{33}, \epsilon_{12}, \epsilon_{23}, \epsilon_{31}\right), \quad \boldsymbol{\sigma}=\left(\sigma_{11}, \sigma_{22}, \sigma_{33}, \sigma_{12}, \sigma_{23}, \sigma_{31}\right),
$$

Equation 2.33 can be rewritten as follows:

$$
\int_{V} \boldsymbol{\sigma} \delta \boldsymbol{\epsilon} d V=\int_{S} p \delta \boldsymbol{u} d \boldsymbol{S}-\int_{V} \delta \boldsymbol{u}[\rho \ddot{\boldsymbol{u}}+\kappa \dot{\boldsymbol{u}}] d V
$$


The internal energy derived from the virtual displacement $\delta \boldsymbol{u}$ in the left hand side of the equation is called thevirtual strain energy and the work derived from $\delta \boldsymbol{u}$ in the right hand side of the equation is called the virtual work.

\section{$2.3 \quad$ Fluid/Structure Interaction}

Although the fluid and the structure do not mix, there exists a fluid/structure interface as a two-dimensional manifold in three-dimensional space. The two disciplines can interact only on the interface itself due to the displacement field determined by the structural system and the pressure field determined by the fluid system. In constructing the governing equations for the continuous system as developed in Sections 2.1.6 and 2.2.2, it is obvious that the displacement and pressure fields must be identical in the representations of the two disciplines. Similarly, in the computation of the aeroelastic system with discrete formulations, these identities must be maintained by some mathematical principles. As examples of such principles, conservation of loads and energy with respect to load transfers from the fluid to the structure, and the geometric conservation law with respect to the deformation tracking from the structure to the fluid are introduced in this section.

\subsubsection{Conservation of Loads and Energy}

In general, while the fluid system addresses the pressure field on the cell surfaces on the interface, the structural system is solved based on a set of concentrated forces at the nodes on the interface. A distributed pressure load, therefore, must be first transfered into equivalent nodal forces. Such a transformation must satisfy two requirements. The first one is that the nodal forces must yield the same net forces as the original distributed pressure loads. Thus, 


$$
\sum_{m} \boldsymbol{f}^{(m)}=\int_{\partial \Omega} p d \boldsymbol{S}
$$

where $\boldsymbol{f}^{(m)}$ is the nodal force vector at the node $m$ in the structural system.

However, an infinite number of possible nodal force sets can easily satisfy this requirement. To determine which of these nodal force sets is the correct one, a second requirement must be introduced, which states the conservation of energy. For the evaluation of the energy, the definition of virtual work is conveniently used so that the term corresponding to the work done by the pressure loads in Equation 2.35 can be replaced by the proper term in formulating the finite element method. Equating the virtual work performed by the nodal forces $\boldsymbol{f}^{(m)}$ acting on a virtual nodal displacement $\delta \boldsymbol{q}^{(m)}$ with that done by the original distributed surface pressure $p$ moving through the equivalent distributed virtual displacement $\delta \boldsymbol{u}$, the second requirement is given as follows;

$$
\sum_{m} \boldsymbol{f}^{(m)} \delta \boldsymbol{q}^{(m)}=\int_{\partial \Omega} p \delta \boldsymbol{u} d \boldsymbol{S} .
$$

Equations 2.36 and 2.37 are what we call conservation of loads and energy.

\subsubsection{Geometric Conservation Law}

In computational aeroelastic applications, the computational domain may be enclosed by moving boundaries. When the boundaries experience motions, there must be at least some part of the computational mesh which moves along the boundary motion. This requires the computation of some geometric quantities that include the grid positions and velocities. These quantities must be evaluated under the enforcement of the so-called geometric conservation law first discussed by Thomas and Lombard [61]. This law states that the computation of the geometric parameters must be performed such that the resulting numerical scheme preserves the state of a uniform 
flow, independently of the mesh motions.

Consider the integral form, Equation 2.23, for an inviscid flow, and let $\Delta t$ and $t^{n}=$ $n \Delta t$ denote respectively the chosen time step and the $n$th time interval. Integration of Equation 2.23 between $t^{n}$ and $t^{n+1}$ leads to

$$
\begin{array}{r}
\int_{t^{n}}^{t^{n+1}} \frac{d}{d t} \int_{\Omega} \boldsymbol{W} d V d t+\int_{t^{n}}^{t^{n+1}} \int_{\partial \Omega} \boldsymbol{F} \cdot d \boldsymbol{S} d t=0, \\
\int_{\Omega} \boldsymbol{W}^{n+1} d V\left(t^{n+1}\right)-\int_{\Omega} \boldsymbol{W}^{n} d V\left(t^{n}\right)+\int_{t^{n}}^{t^{n+1}} \int_{\partial \Omega} \boldsymbol{F} \cdot d \boldsymbol{S} d t=0 .
\end{array}
$$

When $\boldsymbol{W}^{*}$ is assumed to be a given uniform state of the flow, a proposed scheme obviously cannot be acceptable unless it conserves it. Substituting $\boldsymbol{W}^{*}=\boldsymbol{W}^{n+1}=$ $\boldsymbol{W}^{n}$ and $\boldsymbol{F}^{*}=\boldsymbol{F}\left(\boldsymbol{W}^{*}\right)$ in Equation 2.38 , it becomes

$$
\boldsymbol{W}^{*}\left[\int_{\Omega} d V\left(t^{n+1}\right)-\int_{\Omega} d V\left(t^{n}\right)\right]+\int_{t^{n}}^{t^{n+1}} \int_{\partial \Omega} \boldsymbol{F}^{*} \cdot d \boldsymbol{S} d t=0 .
$$

Here the diffusive terms in the flux $\boldsymbol{F}^{*}$ are zero due to the uniformity of the flow. In addition, the convective terms in the flux carried by the fluid velocities are canceled in the spatial integration on the cell boundaries. These two facts can be used to rewrite the integral of the flux as

$$
\int_{\partial \Omega} \boldsymbol{F}^{*} \cdot d \boldsymbol{S}=-\int_{\partial \Omega} \boldsymbol{W}^{*} \boldsymbol{b} \cdot d \boldsymbol{S}
$$

Substituting Equation 2.40 into Equation 2.39 yields

$$
\begin{aligned}
\boldsymbol{W}^{*}\left[\int_{\Omega} d V\left(t^{n+1}\right)-\int_{\Omega} d V\left(t^{n}\right)\right] & =\int_{t^{n}}^{t^{n+1}} \int_{\partial \Omega} \boldsymbol{W}^{*} \boldsymbol{b} \cdot d \boldsymbol{S} d t \\
\int_{\Omega} d V\left(t^{n+1}\right)-\int_{\Omega} d V\left(t^{n}\right) & =\int_{t^{n}}^{t^{n+1}} \int_{\partial \Omega} \boldsymbol{b} \cdot d \boldsymbol{S} d t .
\end{aligned}
$$


In more general terms,

$$
\frac{d}{d t} \int_{V} d V(t)=\int_{S} \boldsymbol{b} \cdot d \boldsymbol{S}(t) .
$$

The resulting equation is called the integral form of the geometric conservation law [62]. This states that the change in volume of each control volume in a certain period must be equal to the volume swept by the cell boundary during that period. Therefore the updating of the boundary coordinates and $\boldsymbol{b}$ cannot be based on mesh distortion issues alone. 


\section{Chapter 3}

\section{Description of the Method}

In order to predict the dynamic response of a flexible structure in a fluid flow, the equations of motion of the structure and the fluid equations must be solved simultaneously. One difficulty in handling the fluid/structure coupling numerically comes from the fact that the structural equations are usually formulated with material (Lagrangian) coordinates while the fluid equations are typically written using spatial (Eulerian) coordinates, in other words, the fluid mesh is fixed in space but the structure mesh is not. If the interface between the two disciplines share a common mesh and the same numerical method is used, combining equations for two displines into a system of equations to provide updated solutions in a single step is still possible with finite element methods, but this approach sometimes suffers ill-conditioning of the matrix for solving the two systems due to the extreme difference in stiffness of the fluid and the structure.

Although the numerical approaches are different from each other, flow solvers and structural solvers have independently matured to the point where they are being used as design tools in industries. Taking advantage of the maturity of both, an alternate approach for a fluid/structure coupled calculation is to combine a flow solver and a structural solver that have been developed in isolation. In such an approach, the 
procedure is advanced in time by solving the flow field and the structural deformation alternatively by independent flow and structural solvers which exchange information on the structural body surface as illustrated in Figure 3.1. The flow solver provides the aerodynamic loads to the structural solver in order for the structural solver to calculate the displacement field of the structure. In return, the structural solver provides the surface deflections to the flow solver which changes the flow fields through the boundary conditions on the structural body surface. In this study, a three-dimensional unsteady RANS flow solver for turbomachinery flows called TFLO [56, 57, 58] and a finite element structural analysis package called MSC/NASTRAN are coupled to perform aeroelastic computations. In the coupling, great attention must be paid to the construction of an interaction procedure between the two disciplines in order not to ruin the accuracy of the combined aeroelastic system. This chapter presents the mathematical descriptions of the computational methods for the unsteady flow solver and the structure solver adapted in this study, the interaction procedure, and finally the parallelization strategy on computations.

\subsection{Unsteady Aerodynamics}

The four major considerations in developing a flow solver for unsteady aerodynamics are the capability to treat flows over complex geometries, proper shock capturing, treatment of viscous effects and computational efficiency. The principles underlying such considerations are now quite well established. In spite of the established principles and the growing computer capabilities, however, direct simulations of the time dependent behavior of eddies are still too computationally costly to be practical. Since the primary purpose of this study is not to understand the details of the flow physics in the boundary layer but to include viscous effects in aeroelastic stability computations, the Reynolds-Averaged Navier-Stokes (RANS) equations are 
TFLO

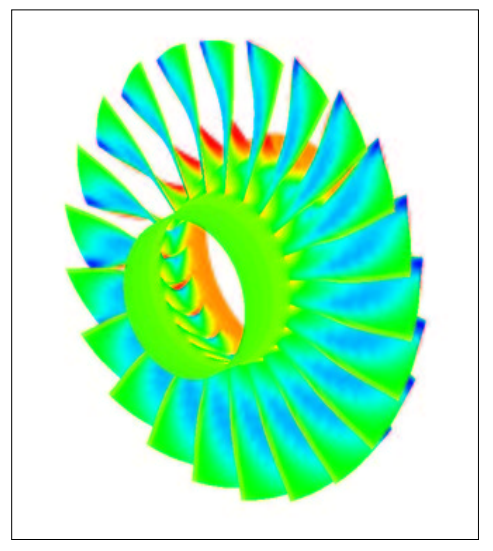

Flow Solver

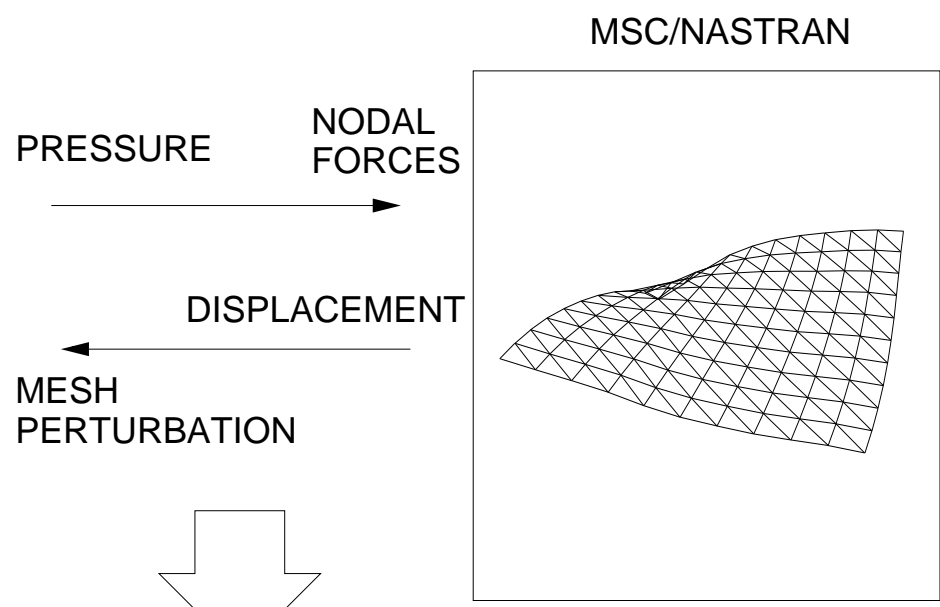

Structural Solver

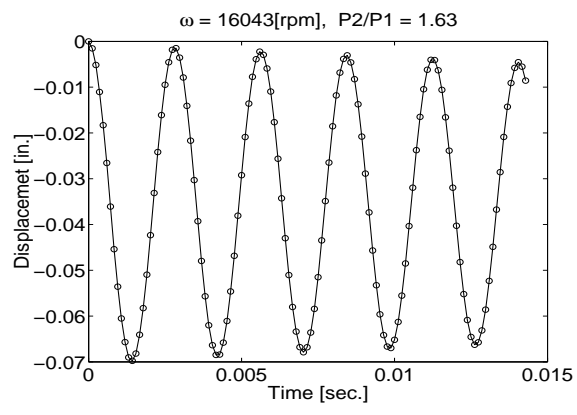

Aeroelastic Response of the Blade

Figure 3.1: Fluid/Structure Coupling 
solved with the introduction of a turbulence model which is discussed later in this section to estimate the effect of turbulence by Reynolds-averaging of the fluctuating components of the viscous flow.

In RANS simulations, the choice of mesh type is of critical importance. In this study, the widely-used structured body-fitted curvilinear meshes are chosen rather than unstructured tetrahedral meshes. Body-fitted structured meshes are well-suited for viscous flow because they can be easily compressed near all solid surfaces. Using a multiblock approach, they are also convenient for discretizing the flow passages in turbomachinery flows with rather straightforward geometries which may, however, include blade tip clearances and relative motion. For computation of unsteady flows around moving body surfaces using these multiblock structural meshes, the cell-centered finite volume scheme is the best choice for reasons discussed later in this section.

In addition to the spatial discretization, there are discussions on two other issues in this section, which should be particularly addressed when using a time-marching method to solve unsteady flows through cascades. The first one is the dual time stepping method, which is one of the cost saving approaches for advancing a equation in physical time with a large time step making use of fast convergence techniques such as multigrid. The second is concerned with the conditions on the periodic boundaries of the blade passage.

These features are all included in the unsteady three-dimensional RANS flow solver for turbomachinery called TFLO, which was originally developed for simulating the flow through multiple compressor or turbine stages. The biggest modification made in TFLO for this study is the addition of the capability to treat a moving mesh system, which is required to ensure that computational grids always fit the blade surfaces as they deform. The modified version of TFLO is combined with a structural solver MSC/NASTRAN to demonstrate the capability for aeroelastic calculations. 


\subsubsection{Cell-Centered Finite Volume Scheme}

A computational domain can be discretized using any of three main methods: finite difference, finite volume, or finite element. Among these, finite volume schemes are the most advantageous for a moving grid because the discretization can be directly made in the physical coordinate system. On the other hand, finite difference schemes can be implemented by means of a transformation from the physical to the computational domain, which requires the evaluation of the Jacobian at every time step when coupled with a moving mesh. Furthermore, combined with a cell-centered discretization, finite volume schemes can integrate the fluxes over each cell more easily to evaluate the time rate of change of the variables at the center of the cell. As for the mesh generation, since passages are rectangular in shape, it is easier to use a structured mesh with hexahedral cells than an unstructured mesh with tetrahedral cells for turbomachinery blade passages. In fact, for unstructured tetrahedral meshes, the finite volume scheme is essentially equivalent to a finite element scheme when a linear shape function is used to approximate the solution. In the present work, the discretization scheme is based on the one proposed by Jameson, Schmidt and Turkel [63]. The procedure is to start from a semi-discrete form of the conservation laws in which only spatial derivatives are numerically approximated, and to proceed with the time integration using a multi-stage time stepping scheme.

The semi-discrete form of the conservation laws for the hexahedral cell shown in Figure 3.2 is obtained as

$$
\frac{d}{d t}\left(V_{i j k} \boldsymbol{W}_{i j k}\right)+\boldsymbol{R}_{i j k}=0,
$$

where $V_{i j k}$ is the cell volume, $\boldsymbol{W}_{i j k}$ is the vector of flow variables evaluated at the center at the cell, and $\boldsymbol{R}_{i j k}$ is the net flux out of the cell. For the cell-centered scheme, this flux can be given as, 


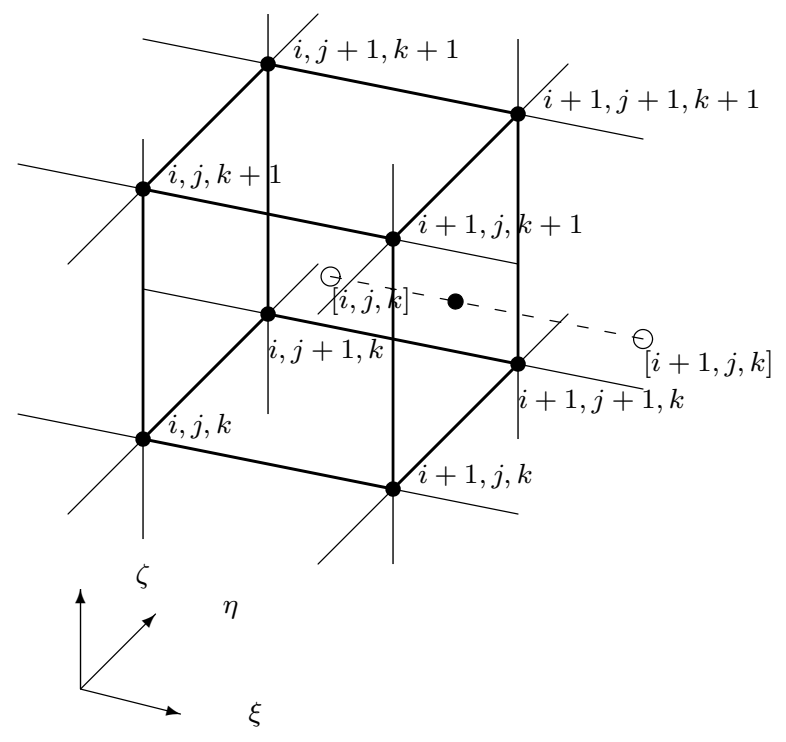

Figure 3.2: Control Volume

$$
\begin{aligned}
\boldsymbol{R}_{i j k} & =\boldsymbol{F}_{i+1 / 2, j, k} \cdot \boldsymbol{S}_{i+1 / 2, j, k}-\boldsymbol{F}_{i-1 / 2, j, k} \cdot \boldsymbol{S}_{i-1 / 2, j, k} \\
& +\boldsymbol{F}_{i, j+1 / 2, k} \cdot \boldsymbol{S}_{i, j+1 / 2, k}-\boldsymbol{F}_{i, j-1 / 2, k} \cdot \boldsymbol{S}_{i, j-1 / 2, k} \\
& +\boldsymbol{F}_{i, j, k+1 / 2} \cdot \boldsymbol{S}_{i, j, k+1 / 2}-\boldsymbol{F}_{i, j, k-1 / 2} \cdot \boldsymbol{S}_{i, j, k-1 / 2},
\end{aligned}
$$

where $\boldsymbol{F}_{i+1 / 2, j, k}$ denotes the values of the flux vector on the surface, and $\boldsymbol{S}_{i+1 / 2, j, k}$ is a vector normal to the face in question with magnitude equal to the area of the face. The flux vector on each face is evaluated simply as the average of the values at the center of the cells on either side of the surface as follows,

$$
\boldsymbol{F}_{i+1 / 2, j, k}=\frac{1}{2}\left(\boldsymbol{F}_{i, j, k}+\boldsymbol{F}_{i+1, j, k}\right)
$$

Here, the use of the centered differences ensures that the scheme is second-order accurate provided that the mesh is sufficiently smooth without any abrupt change in the cell shape and the volume. 


\subsubsection{Artificial Dissipation}

The finite volume scheme described above allows numerical oscillations with alternate signs at neighboring cells. These oscillations can be initiated by discontinuities such as shock waves. One of the methods to eliminate them is to add a sufficient amount of artificial dissipation intentionally to control the generation of spurious oscillations. One of the concepts on which the design of artificial dissipation schemes is based is called Total Variation Diminishing (TVD) proposed by Harten [64]. A dissipation scheme based on TVD ought to be designed so that the total variation of the numerical solution over the whole computational domain cannot increase. Jameson [63] proposed another concept called Local Extrema Diminishing (LED), in which local maxima of the solution cannot increase and local minima cannot decrease. In fact, an LED scheme is also TVD. In this study, a type of artificial dissipation developed by Jameson $[65,66]$ is implemented based on the LED principle. It is called the JamesonSchmidt-Turkel (JST) scheme [63], whose basic idea is to combine monotonicity and higher order accuracy by blending low and high order dissipative terms.

In general, adding artificial dissipation to Equation 3.1, the semi-discrete form can be rewritten as,

$$
\frac{d}{d t}\left(V_{i j k} \boldsymbol{W}_{i j k}\right)+\boldsymbol{R}_{i j k}-\boldsymbol{D}_{i j k}=0,
$$

where, $\boldsymbol{D}_{i j k}$ is the collection of all dissipative terms across all faces of a cell,

$$
\begin{aligned}
\boldsymbol{D}_{i j k} & =\boldsymbol{d}_{i+1 / 2, j, k}-\boldsymbol{d}_{i-1 / 2, j, k}+\boldsymbol{d}_{i, j+1 / 2, k} \\
& -\boldsymbol{d}_{i, j-1 / 2, k}+\boldsymbol{d}_{i, j, k+1 / 2}-\boldsymbol{d}_{i, j, k-1 / 2} .
\end{aligned}
$$

The form of $\boldsymbol{d}_{i+1 / 2, j, k}$ varies depending on the scheme chosen. 


\section{JST scheme}

The JST scheme is a higher order resolution scheme in which higher order diffusive terms are added to the dissipation terms. Higher order terms are anti-diffusive, and may lead to instability if not carefully controlled. For this purpose, pressure sensors are introduced to limit the amount of anti-diffusion in areas of high gradients. Introducing the switch coefficients $\epsilon_{i+1 / 2, j, k}^{(2)}$ and $\epsilon_{i+1 / 2, j, k}^{(4)}$, the face flux for the JST scheme takes the form,

$$
\begin{aligned}
\boldsymbol{d}_{i+1 / 2, j, k} & =\lambda_{i+1 / 2, j, k}\left\{\epsilon_{i+1 / 2, j, k}^{(2)} \Delta \boldsymbol{W}_{i+1 / 2, j, k}\right. \\
& \left.-\epsilon_{i+1 / 2, j, k}^{(4)}\left(\Delta \boldsymbol{W}_{i+3 / 2, j, k}-2 \Delta \boldsymbol{W}_{i+1 / 2, j, k}+\Delta \boldsymbol{W}_{i-1 / 2, j, k}\right)\right\},
\end{aligned}
$$

where $\lambda_{i+1 / 2, j, k}$ is the spectral radius of the Jacobian matrix $A=\frac{\partial \boldsymbol{F}}{\partial \boldsymbol{W}}$ corresponding to the flux through the face $S_{i+1 / 2, j, k}$ defined as,

$$
\lambda_{i+1 / 2, j, k}=\frac{1}{2}\left\{\left|\left(\boldsymbol{u}_{i+1 / 2, j, k}-\dot{\boldsymbol{x}}_{i+1 / 2, j, k}\right) \cdot \boldsymbol{S}_{i+1 / 2, j, k}\right|+c_{i+1 / 2, j, k}\left|\boldsymbol{S}_{i+1 / 2, j, k}\right|\right\},
$$

where $\boldsymbol{u}_{i+1 / 2, j, k}, \dot{\boldsymbol{x}}_{i+1 / 2, j, k}$ and $c_{i+1 / 2, j, k}$ are the velocity vector of the fluid, the velocity vector of the mesh and the speed of sound at the center of the face $S_{i+1 / 2, j, k}$ respectively. $\epsilon_{i+1 / 2, j, k}^{(2)}$ and $\epsilon_{i+1 / 2, j, k}^{(4)}$ in Equation 3.6 control the order of accuracy of the $\boldsymbol{d}_{i+1 / 2, j, k}$ term to produce a low level of diffusion in regions where the solution is smooth, but prevent oscillations near discontinuities. It is necessary to capture shocks sharply and to retain second order accuracy away from the immediate vicinity of the shock wave. The presence of a shock wave is a key factor for determining the proper scale of the dissipative term, and it is sensed by taking the second difference of the pressure as follows, 


$$
\nu_{i j k}=\left|\frac{p_{i+1, j, k}-2 p_{i, j, k}+p_{i-1, j, k}}{p_{i+1, j, k}+2 p_{i, j, k}+p_{i-1, j, k}}\right| .
$$

which is used for $\epsilon_{i+1 / 2, j, k}^{(2)}$ as,

$$
\epsilon_{i+1 / 2, j, k}^{(2)}=\kappa^{(2)} \max \left(\nu_{i+1, j, k}, \nu_{i j k}\right)
$$

It has been found necessary to switch off fourth differences near shocks and this can be done by defining a coefficient

$$
\epsilon_{i+1 / 2, j, k}^{(4)}=\kappa^{(4)}-\epsilon_{i+1 / 2, j, k}^{(2)}
$$

where $\kappa^{(2)}$ and $\kappa^{(4)}$ are constants. Typically,

$$
\kappa^{(2)}=1, \kappa^{(4)}=\frac{1}{32}
$$

For inviscid flows, it should be noted that $\rho H$ should be used rather than $\rho E$ as the fifth component of $\boldsymbol{D}_{i j k}$ in the dissipative terms in order to admit solutions with constant total enthalpy.

\subsubsection{Dual Time Stepping}

Time-marching computations for unsteady flows require the integration of Equation 3.4 to advance the system forward in physical time. The physical time-step used to advance the system in all the cells in the computational domain must be unique, independently of the size of each cell so that the solution at every time step represents a transient solution of the unsteady flow at that point. An explicit time-stepping method, in which the spatial derivatives are calculated from known values of the flow variables at the beginning of the time-step, is certainly an option but it requires the selection of the time-accurate time-step based on numerical stability requirements. A 
restricted time-step results in very high computational cost. An implicit time-stepping method, in which the formulae for the spatial derivatives include the unknown values of the flow variables at the end of the time-step, is in general more costly, but sets less restrictive limitations on the allowable time-step due to numerical considerations. Therefore, it allows the selection of the time-step based on the characteristic frequency of the physical phenomena to be resolved. In this study, the advantages of the large physical time-step of an implicit method and the fast solution techniques developed for an explicit steady-state calculation are included in a time-stepping technique called dual time stepping proposed by Jameson [67].

Introducing a backward difference operator $D_{t}$, Equation 3.4 can be discretized implicitly as follows,

$$
\begin{aligned}
D_{t}\left(\boldsymbol{W}^{(n+1)} V^{(n+1)}\right)+\boldsymbol{R}\left(\boldsymbol{W}^{(n+1)}\right) & =0, \\
\frac{a_{0}}{\Delta t} \boldsymbol{W}^{(n+1)} V^{(n+1)}+\frac{E}{\Delta t}+\boldsymbol{R}\left(\boldsymbol{W}^{(n+1)}\right) & =0
\end{aligned}
$$

where the time level $n \Delta t$ is denoted by the superscript $n$. The operator $E$ represents the part of the time derivative operator that is a function of the values of the flow variables and cell volumes at previous time steps, and is therefore a fixed source term in the solution of each time step. For example, for the second order time backwards discretization,

$$
D_{t}\left(\boldsymbol{W}^{(n+1)} V^{(n+1)}\right)=\frac{3}{2 \Delta t} \boldsymbol{W}^{(n+1)} V^{(n+1)}-\frac{2}{\Delta t} \boldsymbol{W}^{(n)} V^{(n)}+\frac{1}{2 \Delta t} \boldsymbol{W}^{(n-1)} V^{(n-1)},
$$

and

$$
a_{0}=\frac{3}{2}, \quad E=-2 \boldsymbol{W}^{(n)} V^{(n)}+\frac{1}{2} \boldsymbol{W}^{(n-1)} V^{(n-1)} .
$$


The third order time backwards discretization can be shown to be,

$$
\begin{aligned}
D_{t}\left(\boldsymbol{W}^{(n+1)} V^{(n+1)}\right) & =\frac{11}{6 \Delta t} \boldsymbol{W}^{(n+1)} V^{(n+1)}-\frac{3}{\Delta t} \boldsymbol{W}^{(n)} V^{(n)} \\
& +\frac{3}{2 \Delta t} \boldsymbol{W}^{(n-1)} V^{(n-1)}-\frac{1}{3 \Delta t} \boldsymbol{W}^{(n-2)} V^{(n-2)},
\end{aligned}
$$

where

$$
a_{0}=\frac{11}{6}, \quad E=-3 \boldsymbol{W}^{(n)} V^{(n)}+\frac{3}{2} \boldsymbol{W}^{(n-1)} V^{(n-1)}-\frac{1}{3} \boldsymbol{W}^{(n-2)} V^{(n-2)} .
$$

Equations 3.12 can now be regarded as a set of highly nonlinearly coupled ordinary differential equations that can be re-cast into a modified steady-state calculation for every physical time-step by introducing a pseudo time-step $t^{*}$ as follows,

$$
\frac{\partial \boldsymbol{W}}{\partial t^{*}}+\boldsymbol{R}^{*}(\boldsymbol{W})=0
$$

where the modified residual, $\boldsymbol{R}^{*}(\boldsymbol{W})$ contains the usual steady-state residual with the addition of two source terms that arise from the discretization of the time derivative operator,

$$
\boldsymbol{R}^{*}(\boldsymbol{W})=\frac{a_{0}}{\Delta t} \boldsymbol{W}+\frac{1}{V^{(n+1)}}\left[\frac{E}{\Delta t}+\boldsymbol{R}(\boldsymbol{W})\right] .
$$

In Equation 3.17, the solution $\boldsymbol{W}$ can be marched in fictitious time through successive approximations with inner time step $\Delta t^{*}$ to reach a pseudo steady-state which advances the solution forward in time from $t=n \Delta t$ to $t=(n+1) \Delta t$. Once this is accomplished the solution vector $\boldsymbol{W}$ which satisfies Equation 3.17 is actually the new solution at time-step $(n+1), \boldsymbol{W}^{(n+1)}$. Repeating this procedure at every time step, the time accurate behavior of the flow can be predicted as a sequence of pseudo-time steady-state solutions. 
Even if the pseudo time-step $\Delta t^{*}$ is a small number restricted by the numerical stability of the scheme, this method is quite efficient because the convergence to the transient solution at the next physical time step usually does not require as many iterations as a typical steady-state calculation. This is simply based on the fact that the changes in flow variables between the beginning and the end of a physical period is not as drastic as the change from a uniform flow to a steady flow solution.

\subsubsection{Time Marching Scheme}

In solving the ordinary differential equations in 3.17, a time-stepping scheme should be designed solely to maximize the rate of convergence without regard to time accuracy. As discussed in the previous section, the decision to use an explicit scheme with convergence acceleration techniques was made since it is computationally less expensive than using an implicit scheme. The cost of direct inversion of the matrix required in an implicit scheme is so prohibitive that alternative methods such as approximate factorization or iterative method are required. These are essentially equivalent to multi-stage explicit schemes in terms of computational cost. Among multi-stage schemes, a Runge-Kutta scheme is known to allow for the largest timestep [68].

Consider the general semi-discrete equations in 3.17, the general form of an $m$ stage Runge-Kutta scheme is as follows, 


$$
\begin{aligned}
\boldsymbol{W}^{(n+1,0)} & =\boldsymbol{W}^{n}, \\
\vdots & \\
\boldsymbol{W}^{(n+1, k)} & =\boldsymbol{W}^{(n+1,0)}-\alpha_{k} \Delta t^{*} \boldsymbol{R}^{*}\left(\boldsymbol{W}^{(n+1, k-1)}\right), \\
\vdots & \\
\boldsymbol{W}^{n+1} & =\boldsymbol{W}^{(n+1, m)},
\end{aligned}
$$

where the superscript $k$ denotes the $k$-th stage and $\alpha_{m}=1$.

One of the techniques used for faster convergence is called local time stepping, in which fictitious time steps of varying size in different parts of the grid are used to increase the wave speed close to each cell's stability limit, therefore propagating disturbances in the flow faster. Furthermore, in order to optimize the smoothing properties of the scheme, the convective and dissipative parts of the original residual at the $k$ th Runge-Kutta stage $\boldsymbol{R}^{*}$ are treated in a distinct fashion. Thus the residual is split as,

$$
\boldsymbol{R}^{*}\left(\boldsymbol{W}^{(n+1, k-1)}\right)=\boldsymbol{Q}^{(k-1)}+\boldsymbol{D}^{(k-1)},
$$

where $\boldsymbol{Q}$ and $\boldsymbol{D}$ are the convective and dissipative fluxes in the modified residual, respectively. $\boldsymbol{Q}^{(k)}$ and $\boldsymbol{D}^{(k)}$ are defined as,

$$
\begin{aligned}
& \boldsymbol{Q}^{(0)}=\boldsymbol{Q}\left(\boldsymbol{W}^{n}\right), \boldsymbol{D}^{(0)}=\boldsymbol{D}\left(\boldsymbol{W}^{n}\right) \\
& \boldsymbol{Q}^{(k)}=\boldsymbol{Q}\left(\boldsymbol{W}^{(n+1, k)}\right) \\
& \boldsymbol{D}^{(k)}=\beta_{k} \boldsymbol{D}\left(\boldsymbol{W}^{(n+1, k)}\right)+\left(1-\beta_{k}\right) \boldsymbol{D}^{(k-1)} .
\end{aligned}
$$

The coefficients $\alpha_{k}$ are chosen to maximize the stability region along the imaginary 
axis, and the coefficients $\beta_{k}$ are chosen to increase the stability interval along the negative real axis. One of the schemes found to be particularly effective is a 5 -stage scheme with three evaluations of dissipation, whose coefficients are,

$$
\begin{aligned}
& \left\{\alpha_{k}\right\}=\left\{\frac{1}{4}, \frac{1}{6}, \frac{3}{8}, \frac{1}{2}, 1\right\}, \\
& \left\{\beta_{k}\right\}=\{1.0,0.0,0.56,0.0,0.44\} .
\end{aligned}
$$

Since the term, $\frac{a_{0}}{\Delta t} \boldsymbol{W} \mathcal{V}^{(n+1)}$ in the modified residual in Equation 3.18 is only a diagonal term, it can be treated implicitly within the Runge-Kutta integration in fictitious time. Therefore the Runge-Kutta scheme is reformulated as follows;

$$
\begin{aligned}
\boldsymbol{W}^{(n+1,0)} & =\boldsymbol{W}^{n} \\
& \vdots \\
\boldsymbol{W}^{(n+1, k)} & =\frac{1}{\left(1+\alpha_{k} \bar{\lambda}\right)}\left\{\boldsymbol{W}^{(n+1,0)}+\alpha_{k} \bar{\lambda} \boldsymbol{W}^{(n+1, k-1)}-\alpha_{k} \Delta t^{*} \boldsymbol{R}^{*}\left(\boldsymbol{W}^{(n+1, k-1)}\right)\right\} \\
& \vdots \\
\boldsymbol{W}^{n+1} & =\boldsymbol{W}^{(n+1, m)}
\end{aligned}
$$

where $\bar{\lambda}=\frac{a_{0} \Delta t^{*}}{\Delta t}$.

\subsubsection{Multigrid}

The multigrid method, first proposed by Fedorenko [69], is a scheme used to accelerate convergence to the steady-state solution on a fine grid by using corrections resulting from a sequence of solutions obtained on successively coarser grids. The strategy is to accelerate convergence by using large time steps on coarser grids and also to reduce the low frequency error modes by transferring the solution to a coarser grid where the 
error is of higher frequency and can therefore be damped by the relaxation scheme. In the case of an explicit time stepping scheme, a significant amount of computational time can be saved through the possibility of using successively larger time steps on coarser grids without violating the stability conditions [70].

Each coarser mesh is produced by eliminating alternate points of the finer mesh, so that there exists a set of points which are common to all meshes. The cells of the fine mesh can be then combined into larger cells which form a coarser mesh. For unsteady calculations, at every physical time-step, a pseudo-time steady-state problem (Equations 3.17) is solved. The approximation of the state vector, $\boldsymbol{W}_{h}$, is first calculated on the fine mesh, the initial guess of the state vector for the doubled space coarser mesh, $\boldsymbol{W}_{2 h}^{(0)}$, must be made by taking a simple average according to the cell volumes $V_{h}$ over the constituent cells on the finer mesh $(h)$ as follows,

$$
\boldsymbol{W}_{2 h}^{(0)}=\frac{\sum V_{h} \boldsymbol{W}_{h}}{V_{2 h}}
$$

Next, the difference between the aggregated residuals from neighboring points on the fine grid and the residual recalculated on the coarser grid can be defined as a forcing function $P_{2 h}$. In this process, it is necessary to transfer a residual forcing function by simply using the sum of the residuals of the eight cells which make up the new coarse cell.

$$
\boldsymbol{P}_{2 h}=\sum \boldsymbol{R}_{h}^{*}\left(\boldsymbol{W}_{h}\right)-\boldsymbol{R}_{2 h}^{*}\left(\boldsymbol{W}_{2 h}^{(0)}\right)
$$

Then, following the procedure described in the Runge-Kutta integration (Equation 3.23), $\boldsymbol{R}_{2 h}^{*}$ is replaced by $\boldsymbol{R}_{2 h}^{*}+\boldsymbol{P}_{2 h}$. Thus, the multi-stage scheme is reformulated as

$$
\boldsymbol{W}_{2 h}^{(k)}=\frac{1}{\left(1+\alpha_{k} \bar{\lambda}_{2 h}\right)}\left[\boldsymbol{W}_{2 h}^{(0)}+\alpha_{k} \bar{\lambda}_{2 h} \boldsymbol{W}_{2 h}^{(k-1)}-\alpha_{k} \Delta t_{2 h}^{*}\left\{\boldsymbol{R}_{2 h}^{*}\left(\boldsymbol{W}_{2 h}^{(k-1)}\right)+\boldsymbol{P}_{2 h}\right\}\right]
$$


The result $\boldsymbol{W}_{2 h}^{(k)}$ then provides the initial data for the production of a state vector on a coarser grid (4h) based on Equation 3.24, $\boldsymbol{W}_{2 h}$ or the interpolation to the fine mesh $(h) \boldsymbol{W}_{2 h}^{+}$. Finally, the accumulated correction at each coarser level is transfered to the finer level as follows,

$$
\boldsymbol{W}_{h}^{+}=\boldsymbol{W}_{h}+I_{2 h}^{h}\left(\boldsymbol{W}_{2 h}^{+}-\boldsymbol{W}_{2 h}^{(0)}\right),
$$

where $I_{2 h}^{h}$ is an interpolation operator that transfers the corrections from a coarser to a finer mesh. The interpolation operator used in this work amounts to trilinear interpolation.

It has been found that a $W$ cycle illustrated in Figure 3.3 is a particularly effective multigrid strategy. In the figure, $E$ denotes the evaluation of the change in the flow for one step, and $\mathrm{T}$ denotes the transfer of the data without updating the solution. In a $W$ cycle, one time step is advanced on each grid on the way down to the coarser grid, and no residual calculation is performed between the interpolation steps on the way up.

\subsubsection{Boundary Conditions}

In order to maintain the order of accuracy described in Section 3.1.2 in the whole computational domain, the evaluation of the flux through a face requires at least the two adjacent cells in the coordinate direction in which the flux is being evaluated. For purposes of artificial dissipation, it is necessary to add two layers of cells covering the boundary surface of the computational domain as shown in Figure 3.4, so that the calculation of the flux through the boundary face can be treated in exactly the same fashion as any interior cells. These layers of cells are called "halo cells" and the flow variables in these cells must be properly set according to certain boundary conditions. For the calculation of the flow through a cascade in turbomachinery, there are four 


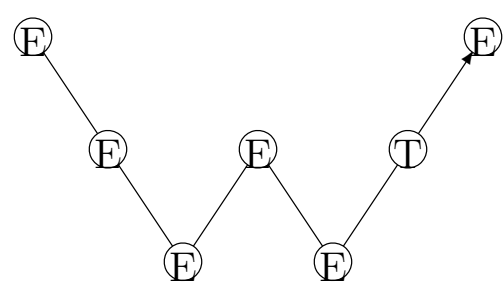

3 Levels

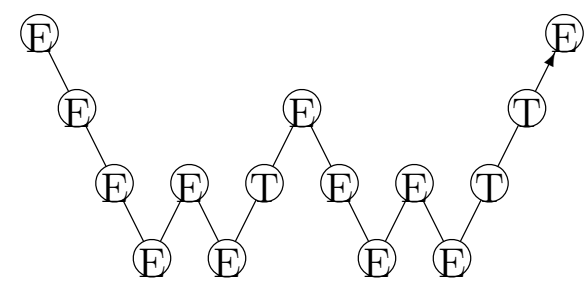

4 Levels

Figure 3.3: Multigrid Cycle

types of boundary conditions that will be discussed in this section and are shown in Figure 3.5. They are the solid wall boundary condition applied on the surface of the blade, the hub and casing surfaces, the inlet boundary condition, the exit boundary condition, and the periodic boundary condition used to take care of the periodicity of turbomachinery flows in the circumferential direction.

\section{Solid Wall}

At a solid wall, the analytic boundary condition that the wall is adiabatic and there is zero normal component of the relative velocity is used. In addition, the tangent component of the relative velocity is zero for a viscous flow. Computationally, this is implemented by simply allowing no mass flux through the solid wall faces and setting the velocity in the halo cells such that flow tangency is enforced on the solid wall for inviscid cases or zero tangent velocity for viscous cases. For a fluid/structure computation in which the surface grid deflects, this simply means that 


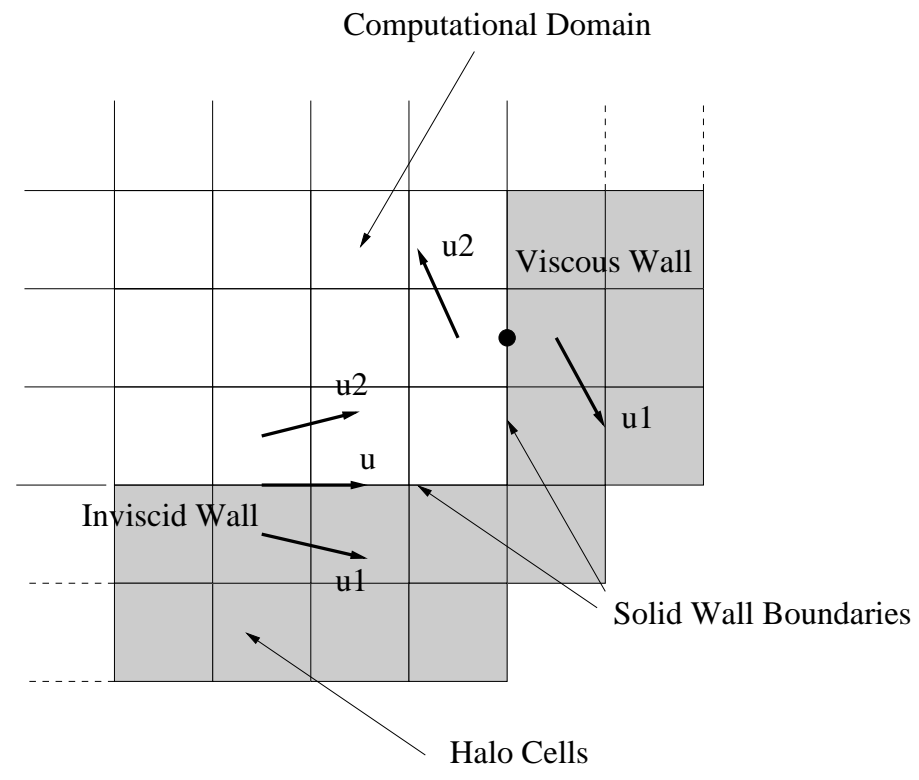

Figure 3.4: Halo Cells and Boundary Condition on Solid Body Surfaces

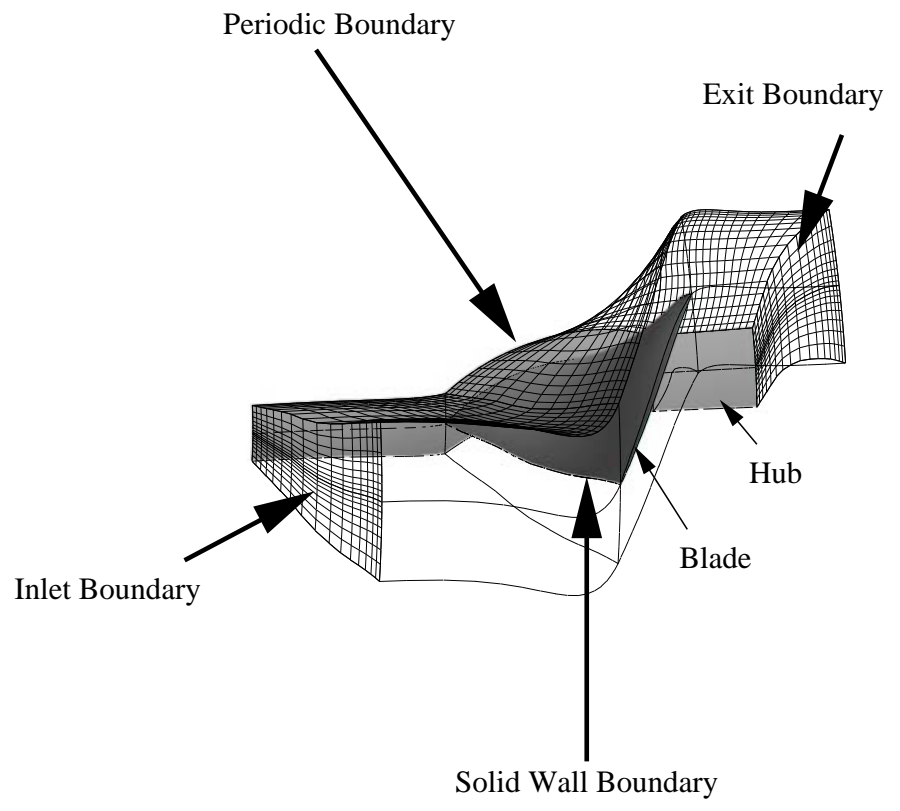

Figure 3.5: Computational Domain and Boundary Conditions for Turbomachinery 


$$
\begin{aligned}
(\boldsymbol{u}-\boldsymbol{b}) \cdot \boldsymbol{n}=0 & \text { for an inviscid flow } \\
\boldsymbol{u}-\boldsymbol{b}=0 & \text { for a viscous flow }
\end{aligned}
$$

where $\boldsymbol{b}$ represents the calculated surface velocity, $\boldsymbol{u}$ represents the fluid velocity in Cartesian coordinates, and $\boldsymbol{n}$ represents the inward unit normal to the boundary surface in question. In a cell-centered finite volume scheme, the value of the velocity at the center of a face is approximated as the average of the cell center values on either side of the face. Therefore, once the value of the flow velocity on the first row of the physical domain on Figure 3.4 is known, the corresponding value in the halo cell 1 can be calculated as follows,

$$
\begin{array}{lr}
\boldsymbol{u}_{1}=\boldsymbol{u}_{2}-2[(\boldsymbol{u}-\boldsymbol{b}) \cdot \boldsymbol{n}] \boldsymbol{n} & \text { for an inviscid flow, } \\
\boldsymbol{u}_{1}=-\boldsymbol{u}_{2} & \text { for a viscous flow. }
\end{array}
$$

This condition fixes the value of three of the five independent flow variables $(\rho, p, u, v, w)$ inside the halo cells. It is common to fix the value of the density, $\rho_{1}$, within the halo cell equal to the density $\rho_{2}$ in the cell adjacent across the boundary. Thus the remaining dependent flow variable at an solid wall boundary now becomes pressure and it provides the only contribution to the flux balance in practice. Using the four known values, three Cartesian components of the momentum equation can be rewritten at the solid wall boundary to yield the pressure gradient in the direction normal to the wall. Using the known pressure at the interior cell directly above the solid wall, the pressure in the halo cell can then be estimated by extrapolation from the adjacent cell center with the known normal pressure gradient at the wall. In the actual computation, a simple zeroth order linear extrapolation of the pressure from 
the cell directly above of the halo cell is used to avoid any error that may arise from higher order extrapolation. Thus, the values of five independent variables for ghost cells at the solid wall boundary are now computed.

In addition to pressure, boundary conditions are also needed for temperature for the Navier-Stokes equations. For a high Reynolds number flow, the pressure gradient normal to the wall is approximately zero. Together with the essential adiabatic wall condition in which the temperature gradient normal the wall is also zero, these conditions can be described as,

$$
\frac{\delta p}{\delta n}=0, \quad k \frac{\delta T}{\delta n}=0 .
$$

As noticed, the temperature gradient in the wall normal direction at an adiabatic wall is automatically kept zero as long as the simple extrapolation of density and pressure is applied.

\section{Inlet and Exit}

Five independent variables must be given at each inlet and exit boundary to solve the flow governing equations in three-dimensions. The number and type of conditions that need to be specified from information extended to the flow, as well as that which must be calculated from information from the interior flow itself, can be determined from an examination of the characteristic paths bringing information to each boundary cell.

At the inlet boundary, four of the five independent flow variables must be specified for axially subsonic inlet flows. Similarly to actual experimental setups, total pressure, total temperature and two independent flow angles of the incoming flow are fixed. The other flow variable must be extrapolated from the interior flow field according to a characteristic analysis. The one-dimensional Riemann invariant normal to the inlet boundary is used to determine the other variable in the corresponding halo cell. The 
outgoing one-dimensional characteristic equation can be written as,

$$
V_{n}+\frac{2}{\gamma-1} c=V_{n, \infty}+\frac{2}{\gamma-1} c_{\infty}
$$

where the subscript $\infty$ denotes free upstream values and $V_{n}$ denotes the velocity component in the direction normal to the inlet boundary, and $c$ is speed of sound. Solving this equation for $c$, all the flow variables on the halo cells adjacent to the inlet boundary can be calculated. For supersonic inlets, the values of all five flow variables are specified.

On the other hand, only the pressure is specified at the exit boundary for subsonic flows. The other variables on the halo cells adjacent to the exit boundary are determined by simple extrapolation of density and three components of velocity from the interior cells adjacent to the boundary for the Navier-Stokes equations. For supersonic outlet flows, all variables are extrapolated from the interior flow.

\section{Periodic Boundary}

For steady and unsteady flow cases with equal wake and rotor pitches, the periodic boundary condition states that the flow on one circumferential boundary is exactly the same as the flow at the corresponding point on the other circumferential boundary at the same time. Thus, in the cylindrical coordinate system,

$$
\boldsymbol{W}(x, r, \theta, t)=\boldsymbol{W}\left(x, r, \theta+\theta_{P}, t\right),
$$

where $x, r, \theta$ are the components of cylindrical coordinates and $\theta_{P}$ denotes the cascade angular pitch. Computationally, it is implemented by imposing the updated flow variables that one interior cell on the circumferential boundary obtains at the halo cell center of the corresponding periodic circumferential boundary. As a result, the flow variables at both cells can be updated using the regular differentiation so that 
the solution is identical at the two circumferential boundaries.

Although no inter-blade phase angle can be ideally assumed for a single blade row without any upstream disturbance, for most unsteady flows, adjacent blades usually can be assumed to vibrate with an approximately constant phase difference. When the inter-blade phase angle is not zero, the computational domain has to be extended to multi-passage or a phase-shifted periodic boundary condition has to be applied if the computation is performed in a single blade passage. Some methods have been proposed below for phase-shifted periodic boundary conditions.

The direct store method $[31,30]$, originally developed for blade row interaction problems, is the first method to handle these kinds of boundaries. In this method, variables of state vectors at the periodic boundaries are stored at every time step for a period of oscillation. The time-marching process continues to a periodic solution, in which the stored values for a vibration period at one circumferential boundary are essentially the same at the other circumferential boundary but phase-shifted in time. As a result, large computer storage is required for this technique.

L. He [71] proposed a method called "shape correction." Flow variables at the periodic boundaries are Fourier transformed. Instead of storing flow variables over the appropriate temporal period as in the direct store method, only a subset of the Fourier components of the series function fitted to the temporal profile are stored, so that computer storage is greatly reduced. The stored temporal "shape" of the flow variables is then used to correct the current solution at the periodic boundaries. At periodic boundaries, all the perturbations are identified by their own phase-shifted periodicities and approximated by Fourier series.

In this study, structural computations are coupled which may not necessarily produce exactly the same structural motions of adjacent blades. This possibility forces multi-passage computations to be performed. The number of passages needed depends on the inter-blade phase angle of the initial excitation that is enforced by 
the initial deflection of the blade before the aeroelastic coupling starts.

\subsubsection{Turbulence Model}

Most turbulence models used for engineering flows follow the Boussinesq hypothesis, that is, they assume that the Reynolds shear stress is directly proportional to the mean strain rate in a way similar to the laminar shear stress but using $\mu_{t}$ instead of $\mu$,

$$
-\overline{\rho u_{i}^{\prime \prime} u_{j}^{\prime \prime}}=\mu_{t}\left[\frac{\partial \tilde{u}_{i}}{\partial x_{j}}+\frac{\partial \tilde{u}_{j}}{\partial x_{i}}\right]-\frac{2}{3} \mu_{t}\left[\frac{\partial \tilde{u}_{k}}{\partial x_{k}}\right] \delta_{i j},
$$

where $\mu_{t}$ is the apparent eddy viscosity caused by the turbulent momentum transfer. The Reynolds heat flux is also assumed to be modeled by the Fourier law similarly to the laminar viscosity as follows,

$$
-\overline{\rho e^{\prime \prime} u_{j}^{\prime \prime}}=-k_{t} \frac{\partial T}{\partial x_{j}}, \quad k_{t}=\frac{c_{p} \mu_{t}}{\operatorname{Pr}} .
$$

The turbulent eddy viscosity $\mu_{t}$ has units of density times length times velocity,

$$
\mu_{t} \propto \rho L U
$$

In the actual calculations, the local fluid density can be used for the density factor in this equation. The remaining scales of the local turbulent motion, a turbulence length scale $L$ and a turbulence velocity $U$, need to be modeled.

There are several main categories of turbulence models: algebraic (zero-equation) models, one-equation models, two-equation models, etc. By definition, an $n$-equation model represents a model that requires the solution of $n$ additional differential transport equations. The Baldwin-Lomax model [43] is the most widely used algebraic turbulence model. Algebraic models are simple because they calculate turbulent viscosity directly from mean flow quantities. However, that causes a weakness associated 
with algebraic turbulence models, which is that they require extensive fine tuning on grid smoothness and orthogonality to the surface to predict turbulent viscosities accurately especially in regions of separation and wakes. In addition, the Baldwin-Lomax model encounters a difficulty in determining the location of the boundary layer edge when it is used for flows surrounded by two intersecting walls. Calculation of the convection, production, and dissipation of turbulence quantities associated with the flow using one- or two-equation turbulence models provides more physically realistic determinations of turbulent viscosities to overcome these weaknesses. One-equation models are incomplete, however, in the sense that they relate the turbulence length scale to some typical flow dimension. Of one-equation models, the Spalart-Allmaras model [72] is the most popular. The model has been applied to industrial turbomachinery applications because of its significant improvement over algebraic models and other one-equation models which comes from the calibrations with several kinds of empirical data. It however still requires the calculation of the distance to the nearest wall, which causes the similar difficulty to the Baldwin-Lomax model when it is applied to flows surrounded by two intersecting walls. On the other hand, two-equation models construct equations for the turbulence length scale or its equivalent, and thus they are complete. One of the most popular two-equation model is the $k-\omega$ model developed by Wilcox [73], in which two equations are solved for turbulent kinetic energy $k$ and specific dissipation rate $\omega$. Two-equation models are superior for massively separated flows. TFLO includes these three options: the Baldwin-Lomax model, the Spalart-Allmaras model and the $k-\omega$ model.

In this study, the $k-\omega$ model is found to be the most suitable choice for following reasons. One of the the focus of this study is directed at transonic flows with a high rotation speed in which massive separations due to shock/boundary layer interactions or vortex sheddings due to the tip leakage flows are expected. Although two-equation models are computationally more expensive and require finer grids near the wall, the 
$k$ - $\omega$ model is considered to be the most accurate among the implemented models for such complicated viscous transonic flows with timely changing grids in this particular study. The following section provides the description for the model in specific details.

\section{Wilcox $k-\omega$ Model}

In the Wilcox $k$ - $\omega$ model, the variables which are related to the turbulence scale length and the turbulence velocity in Equation 3.37 are the turbulent kinetic energy per unit mass $k$ and the specific dissipation rate $\omega$ as mentioned. They are defined and related to the turbulent eddy viscosity $\mu_{t}$ as,

$$
\mu_{t}=\alpha^{*} \frac{\rho k}{\omega}, \quad k \equiv \frac{1}{2} \overline{\rho u_{i}^{\prime \prime} u_{i}^{\prime \prime}}
$$

$k$ and $\omega$ are determined to satisfy the following two transport equations.

$$
\begin{aligned}
& \frac{\partial}{\partial t}\left[\begin{array}{c}
\rho k \\
\rho \omega
\end{array}\right]+\frac{\partial}{\partial x_{j}}\left[\begin{array}{c}
\rho k \\
\rho \omega
\end{array}\right]\left(u_{j}-b_{j}\right) \\
& =\left[\begin{array}{c}
\tau_{i j}^{t} \frac{\partial u_{i}}{\partial x_{j}} \\
\omega \frac{\omega}{k} \tau_{i j}^{t} \frac{\partial u_{i}}{\partial x_{j}}
\end{array}\right]-\left[\begin{array}{c}
\beta^{*} \rho \omega k \\
\beta \rho \omega^{2}
\end{array}\right]+\frac{\partial}{\partial x_{j}}\left[\begin{array}{c}
\left(\mu+\sigma^{*} \mu_{t}\right) \frac{\partial k}{\partial x_{j}} \\
\left(\mu+\sigma \mu_{t}\right) \frac{\partial \omega}{\partial x_{j}}
\end{array}\right],
\end{aligned}
$$

where the first, second and third terms in the right hand side of the equations are the production, dissipation and diffusion terms, respectively. The viscous stress tensor $\tau_{i j}^{t}$ in the production term is based on the turbulent eddy viscosity which replaces the laminar viscosity in Equation 2.9. Closure coefficients for the model are calibrated as follows, 


$$
\begin{aligned}
\alpha & =\frac{\beta-\sigma \kappa^{2} \sqrt{\beta^{*}}}{\beta^{*}}, & \alpha^{*} & =1, \\
\beta & =3 / 40, & \beta^{*} & =9 / 100, \\
\sigma & =1 / 2, & \sigma^{*} & =1 / 2,
\end{aligned}
$$

where the coefficient $\kappa$ is known as the Karman constant which is approximately equal to 0.41. Thus Equations 3.39 are closed at each field point to yield the values for $k$ and $\omega$ with a proper numerical solution procedure.

It should be also noted that the $k-\omega$ model requires the total energy and the enthalpy in Equations 2.24 used in RANS equations to be redefined as follows,

$$
E=e+k+\frac{u_{i} u_{i}}{2}, \quad H=h+k+\frac{u_{i} u_{i}}{2},
$$

where $h=e+p / \rho$.

In the numerical solution procedure of the RANS solver, the two equations for the $k$ - $\omega$ model are solved segregated from the mean flow. They are discretized using a cell-centered finite difference scheme and advanced in time using an implicit time integration scheme. For three-dimensional flow, the implicit operators are approximately factored into three tridiagonal matrices and inverted independently using an Alternation Direction Implicit (ADI) scheme. In constructing the matrices, the production and dissipation terms are treated explicitly while the convection and diffusion terms are linearized for the implicit operators. The turbulence model is updated after each complete Runge-Kutta time update on the finest mesh of the multigrid cycle, but the turbulence variables is frozen on the coarser meshes.

\subsubsection{Moving Mesh System}

For an aeroelastic analysis with computational fluid dynamics in a time-marching fashion, it is necessary to deform the fluid mesh at each physical time step so that 
it continuously conforms to the instantaneous shape of the aeroelastically deforming body under consideration. In the mesh generation for complex three-dimensional turbomachinery configurations, elliptic or hyperbolic partial differential equations may often be solved in an iterative fashion to obtain acceptably smooth meshes. However, these iterative mesh generation procedures at each physical time step can be computationally expensive as a whole when they are applied to a dynamic aeroelastic application.

Robinson et al. [74] developed and implemented a simple moving mesh algorithm without the iterations. In his method the mesh is modeled as a spring network, where each edge of each hexahedral cell represents a linear spring. The stiffness of each spring is inversely proportional to a specified power of the length of the edge. This method works well with finely turned meshes but in theory it could produce tangles in skew cells. Another issue is brought about by the fact that, for most of the mesh, especially away form the structural body surfaces, mesh perturbations are not required. To avoid unnecessary perturbations of meshes and take advantage of the quality of the base line mesh throughout the entire aeroelastic calculation, the zonal moving mesh technique, in which only the sub-meshes in local regions around oscillating blades for a turbomachinery case are moved was proposed by L. He[30].

In the present study, a high quality mesh of the whole computational domain is divided into an appropriate multiblock system for parallelization purposes. This initial multiblock mesh may become the basis for all subsequent meshes that are obtained by a simple analytical perturbation. A similar concept to the zonal moving mesh method can be used for multiblock meshes because it is often sufficient to perturb only blocks in direct contact with the deformed structural body surfaces. Because the flow solver assumes a point-to-point match between adjacent blocks in the mesh, each block may be independently perturbed, provided that modified surfaces are treated continuously across block boundaries. The entire method of perturbing an 

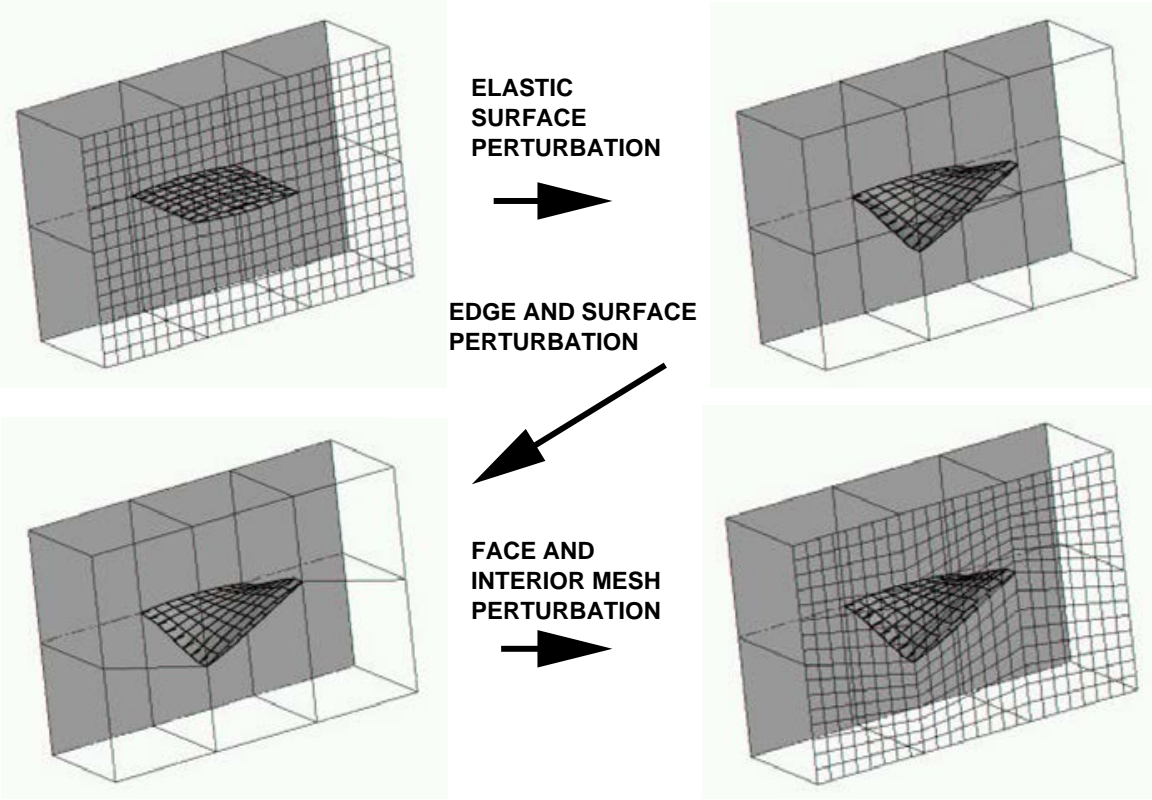

Figure 3.6: Moving Mesh Procedure

existing multiblock mesh to create a new one for the next time step takes a four-stage procedure as shown in Figure 3.6. First, all block faces that are directly affected by the structural motion of the body are explicitly perturbed by the deformation tracking algorithm described in Section 3.3.1. Secondly, all edges that are in contact with a face of the structural body, either in the same or in an adjacent block, are implicitly perturbed with the algebraic method described below. Next, all faces that either include an implicitly perturbed edge or adjacent to a structural body face are implicitly perturbed with an algebraic method for each index line. Finally, the interior points belong to all blocks that have one or more explicitly or implicitly perturbed faces are perturbed in the same way as the edge and face perturbations.

For edge, face and block perturbation in these procedures, the arc-length attenuation algorithm developed by Reuther et. al. [75] is used because it is mathematically very simple but can avoid tangles of the meshes and maintain high mesh quality. In this algorithm, all the original mesh points are first transfered to another coordinate 
system where each component is related to its arc length along the index line that the mesh point lies, and then normalized with the total arc length of that index line. Once both ends of the index line are perturbed, then all the mesh points in between are moved by an amount which is attenuated by the arc length, thus always maintaining their transfered normalized coordinates. According to the entire procedure described above, the second stage shifts the eight corner points of the block in question and corrects the perturbations of the twelve edges of the block resulting from the corner shifts to attain the final desired edge locations. The third stage corrects the perturbations of the six faces surrounded by these edges. Finally, the fourth stage corrects the interior points to produce the desired new mesh with face point motions accounted for.

As far as the geometric conservation law is concerned, this moving mesh procedure combined with the dual time stepping keeps the same order of accuracy in time as the time derivative operator described in Section 3.1.3. The geometric conservation law given in Equation 2.42 can be applied to each cell of the computational domain. Although the boundary velocity of a cell on the structural surface can be transfered from the node velocities of the structural model by the deformation tracking system in Section 3.3.1, the determination of the boundary velocity of an interior cell needs to rely on the discretization of the grid positions in time using the time derivative operator. Thus, the left hand side of Equation 2.42 results in an approximation with the same order of accuracy in time as the time derivative operator. In fact, this approach, however, does not affect the accuracy of the unsteady computation. As discussed later, the accuracy in time of the coupled system is as high as first order which would be less than the accuracy of the geometric conservation law if the second or third order derivative was used.

Although this mesh perturbation algorithm for multiblock meshes is quite sophisticated for most external flow applications, it is not inevitable that the method might 
produce poor mesh qualities when large perturbations happen within a small block. In turbomachinery, any mesh topology for a multiblock system is forced to have at least one block which represents the tip clearance region. This block usually has the stationary casing surface on one face and the tip section of the blade or an implicitly perturbed face by the motion of the tip on the opposite face. Furthermore the length of this kind of block in the radial direction is very short but the deflections at the tip of the perturbed faces in the circumferential direction is fairly large. As a result, the short edges in the radial direction will become oblique as illustrated in Figure 3.7. This poor quality of the mesh in the tip clearance region may fatally affect accuracy and stability of the computation because the flow complexity around this region is severe due to the transonic flow and vortex shedding at the tip.

To overcome this difficulty, another mesh perturbation procedure for this particular region must be introduced. Instead of keeping the mesh points on the casing stationary, they are allowed to slide along the casing surface such that the blocks representing the tip clearance maintain their mesh quality as much as possible. The arc-length attenuation method is also used in this process. The procedure taken in this study is as follows. First, the faces on the blade or the implicitly perturbed faces are perturbed according to the aeroelastic motion of the blade. Second, the leading edge and the trailing edge of the tip section are extended along each edge line in the span-wise direction to the casing in order to detect the crossing point on the casing using some interpolations. Once the corner points of the block on the casing are determined, the next step is to move the edges, faces, and interior points of the block for the tip clearance region according to the arc-length attenuation method. Note that when the edges and faces on the casing are perturbed, the points on the casing are forced to lie along the original casing surfaces using interpolations.

Figures 3.8 and 3.9 show how a block surface is perturbed using the usual block perturbation and the improved procedure for the tip clearance, respectively. The 

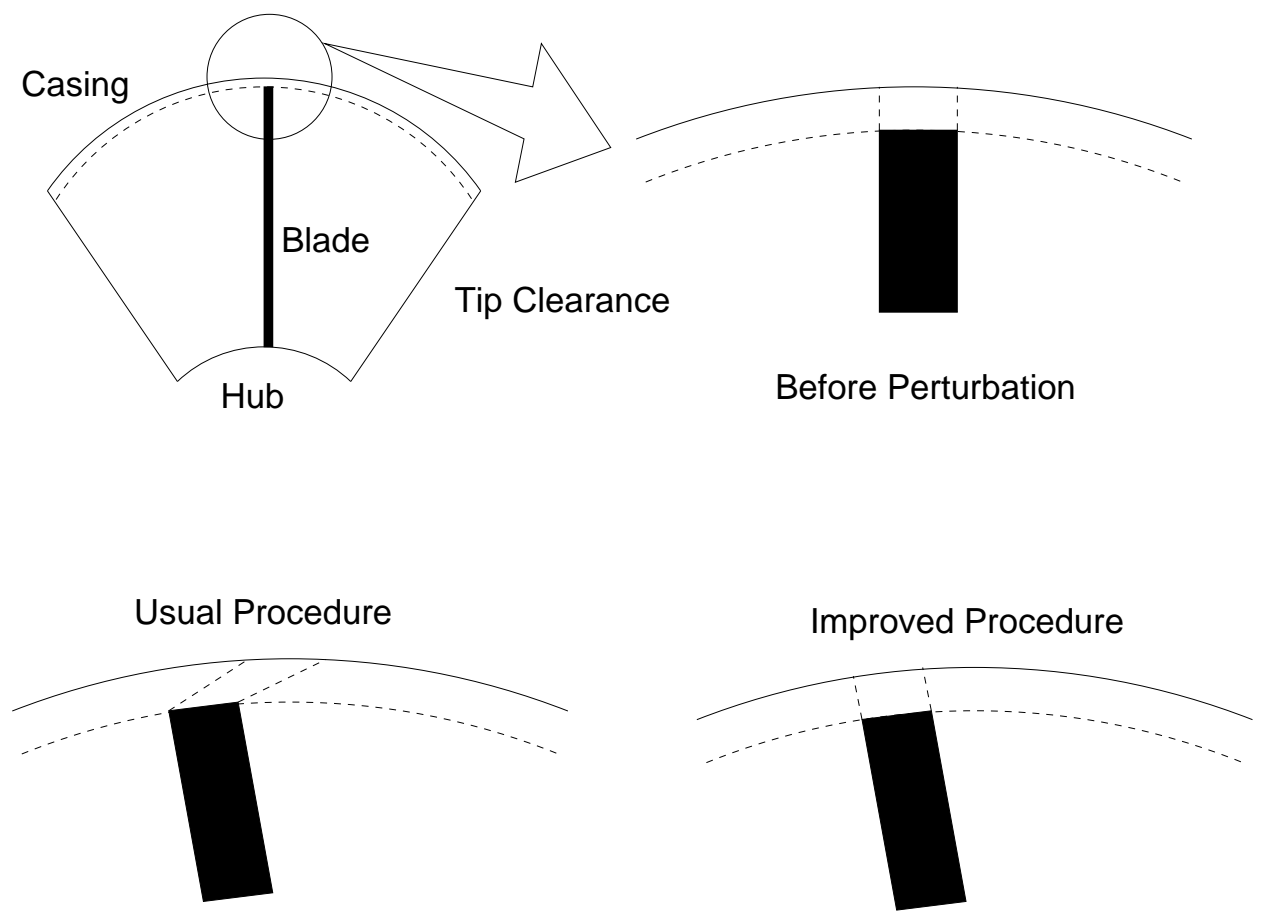

After Perturbation

Figure 3.7: Moving Mesh Procedure in the Tip Clearance Region

figures show the view of the trailing edge at the tip of the blade looking inward radially from the casing. The meshed and solid surfaces in the figures represent the original and new location of the blade, respectively. Without the improved procedure, the block for the tip clearance is sheared by the tip motion of the blade and the stationary casing resulting in the poor quality of the interior cells. On the other hand, the improved procedure creates the new mesh as if the blade were extended through the casing such that the original mesh quality of the block for the tip clearance can be maintained. 


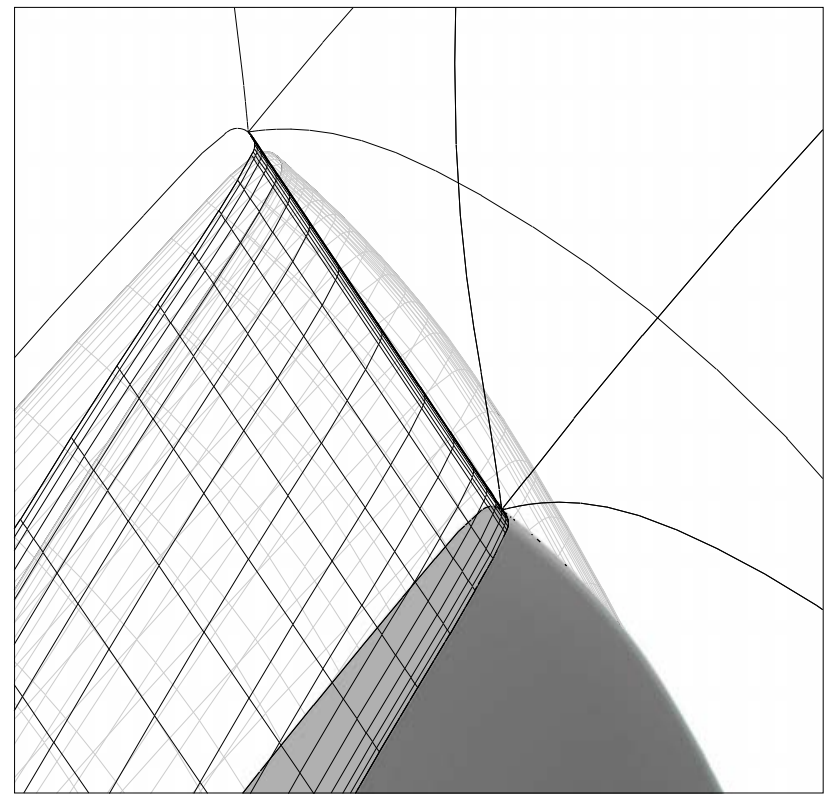

Figure 3.8: Perturbed Mesh for the Tip Clearance Using the Usual Procedure

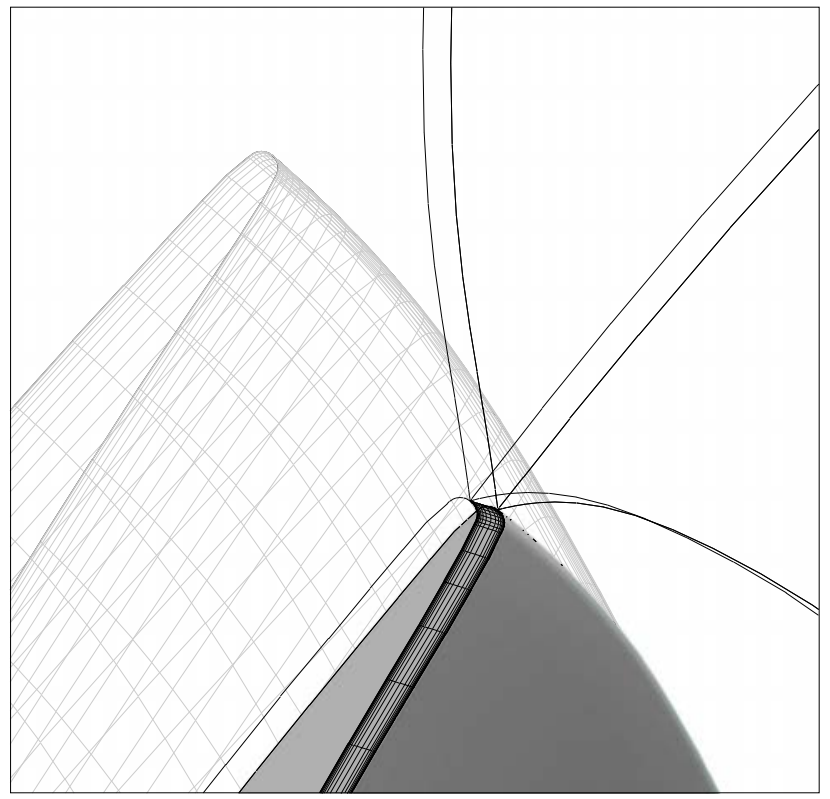

Figure 3.9: Perturbed Mesh for the Tip Clearance Using the Improved Procedure 


\subsection{Structural Mechanics}

In the classical linear theory for aeroelastic problems, the structural model can be reduced to a small degree of freedom eigenvalue problem to solve for the amplitude of each degree of freedom. The earliest structural model was probably the Typical Section [47] with two degrees of freedom, plunging and pitching motions of each blade. However, if a nonlinear aerodynamic model is used, it is generally more efficient to perform aeroelastic analysis in the time domain, by integrating the fluid/structure coupled equations providing more accurate structural responses.

For an independent structural analysis of turbomachinery blades, researchers traditionally have modeled blades as one-dimensional straight, slender, twisted, elastic beams, with symmetric varying cross sections, based on a continuum beam method such as the Rayleigh-Ritz method. However, when the blade span is small and sufficiently wide along its chord, it behaves more like a plate or shell rather than a beam. The classical beam theory is not valid anymore as it cannot predict plate modes. Modern fan and compressor blades are thin and have a considerable amount of built-in pre-twist especially for fan blades. In addition, rotor blades are inertially coupled by operation in a strong rotational body force field. The flat-plate theory [76] and the thin shell theory with camber, twist and rotation [77] have proven useful with the Rayleigh-Ritz method in determining the mode characteristics of blades. While energy methods such as the Rayleigh-Ritz method require a lot of analytical background work, a finite element model is relatively simple but able to represent more complex blade structure accurately. As far as the interaction between the fluid system and the structural system is concerned, a finite element model is more convenient because the local displacements and loads can be represented at adequately distributed nodes which can simply communicate variables with the close mesh points 
in the fluid system. In this study, an industry standard finite element analysis package, MCS/NASTRAN, is used for its reputation in capability and accuracy and its large library of elements.

Some researchers also addressed the importance of modeling the rotor structure as a bladed-disk assembly [78]. The bladed-disk assembly exhibits the same vibration properties as a simple disk, but the relative disk and blade flexibilities determine the overall characteristics of the vibration. For long-span wide-chord fans, the disk is usually much stiffer than the blade, hence assembly modes are dominated by the blade characteristics [79]. Since such fans are the main interest in this study because they are the most critical in terms of flutter boundaries, rotor disks are not included in the structural modeling. Modeling a bladed-disk assembly would be more realistic but it would require flow calculations with the whole wheel of the rotor, which would be computational quite expensive and not expected to give more accuracy.

In this section, fundamentals of the finite element analysis are discussed followed by discussions on the modeling of turbomachinery blades especially about the choice of the finite element, damping characteristics and the inclusion of centrifugal and Coriolis forces. Finally a numerical method for integrating the structural equation in time to calculate nodal displacements at each period is discussed.

\subsubsection{Finite Element Analysis}

In structural finite element analysis [80, 81], a body is approximated as an assembly of discrete finite elements interconnected at nodal points on the element boundaries. The material displacement measured in a local coordinate system $(x, y, z)$, within each element is assumed to be a function of the displacements at nodes of the finite element. For element $m$, the displacements $\boldsymbol{u}^{(m)}$ at a point in space $(x, y, z)$ is written as, 


$$
\boldsymbol{u}^{(m)}(x, y, z)=\boldsymbol{H}^{(m)}(x, y, z) \boldsymbol{q},
$$

where $\boldsymbol{H}^{(m)}$ is the displacement interpolation matrix, the superscript $m$ denotes element number $m$, and $\boldsymbol{q}$ is the vector of the six global displacements at all the nodal points of element $m$. The six components basically include translational components in the three Cartesian directions and rotation components about the same axes if elements with rotational degrees of freedom are considered. The entries in $\boldsymbol{H}^{(m)}$ depend on the element geometry, the number of element nodes and degrees of freedom, and the element parameterization chosen. This relation is appropriate for small displacements of the structure but may lead to severe grid distortions when the structure undergoes large deflections. With the assumption that the displacements can be represented by Equation 3.42, the corresponding strain can be evaluated using the strain-displacement relations in Equations 2.30.

$$
\boldsymbol{\epsilon}^{(m)}(x, y, z)=\boldsymbol{B}^{(m)}(x, y, z) \boldsymbol{q},
$$

where $\boldsymbol{B}^{(m)}$ is the strain-displacement matrix which is obtained by differentiating $\boldsymbol{H}^{(m)}$. Furthermore, the stresses in the finite element are related to the element strain by the strain-stress relations in Equations 2.31.

$$
\boldsymbol{\sigma}^{(m)}=\boldsymbol{C}^{(m)} \boldsymbol{\epsilon}^{(m)},
$$

where $\boldsymbol{C}^{(m)}$ is the elasticity matrix of element $m$ and its components are given in Equations 2.31. The material property specified in $\boldsymbol{C}^{(m)}$ for each element can be assumed to be that for an isotropic material and to depend on the type of the element. Using these assumptions, the principle of virtual work in Equations 2.35 for the assembly of finite elements is now considered and rewritten as a sum of integrations over the volume and area occupied by all finite elements, 


$$
\begin{aligned}
\sum_{m} \int_{V(m)} \delta \boldsymbol{\epsilon}^{(m) T} \boldsymbol{\sigma}^{(m)} d V^{(m)} & =\sum_{m} \int_{V(m)} \delta \boldsymbol{u}^{(m) T}\left[-\rho^{(m)} \ddot{\boldsymbol{u}}^{(m)}-\kappa^{(m)} \dot{\boldsymbol{u}}^{(m)}\right] d V^{(m)} \\
& +\delta \boldsymbol{q}^{T} \cdot \boldsymbol{f},
\end{aligned}
$$

where $\boldsymbol{f}$ is a global vector of concentrated loads applied to the finite element assembly. Notice that the $i$ th component in $\{\boldsymbol{f}\}$ is the concentrated nodal force that corresponds to the $i$ th displacement component in $\{\boldsymbol{q}\}$. The last term in this equation, the work done by the aerodynamic force, is computed using the conservation of energy principle discussed in Section 2.3.1. These loads must also be equivalently transfered from the external aerodynamic forces applied to the structure according to the conservation of loads. The method of transformation is discussed in Section 3.3.2. Substitution of 3.42, 3.43 and 3.44 into Equation 3.45 yields,

$$
[\boldsymbol{M}]\{\ddot{\boldsymbol{q}}\}+[\boldsymbol{C}]\{\dot{\boldsymbol{q}}\}+[\boldsymbol{K}]\{\boldsymbol{q}\}=\{\boldsymbol{f}\},
$$

where

$$
\begin{aligned}
{[\boldsymbol{M}] } & =\sum_{m} \int_{V(m)} \rho^{(m)} \boldsymbol{H}^{(m) T} \boldsymbol{H}^{(m)} d V^{(m)}, \\
{[\boldsymbol{C}] } & =\sum_{m} \int_{V(m)} \kappa^{(m)} \boldsymbol{H}^{(m) T} \boldsymbol{H}^{(m)} d V^{(m)}, \\
{[\boldsymbol{K}] } & =\sum_{m} \int_{V(m)} \boldsymbol{B}^{(m) T} \boldsymbol{C}^{(m)} \boldsymbol{B}^{(m)} d V^{(m)} .
\end{aligned}
$$

$[\boldsymbol{M}],[\boldsymbol{C}]$ and $[\boldsymbol{K}]$ are the mass, damping and stiffness matrices, respectively. In practice it is difficult, if not impossible, to determine the element damping parameters in $[\boldsymbol{C}]$ for general finite element assemblies. The damping characteristics of the structure are discussed in Section 3.2.3 in more detail.

In a time-marching aeroelastic analysis, once the matrices $[\boldsymbol{M}],[\boldsymbol{C}],[\boldsymbol{K}]$ and the 
load vector $\{\boldsymbol{f}\}$ are calculated, the equations of motion 3.46 can be integrated in time starting from initial conditions for the vectors $\{\boldsymbol{q}\}$ and $\{\dot{\boldsymbol{q}}\}$. Thus the structural response given by the time history of the displacement vector $\{\boldsymbol{q}\}$ can be obtained.

MSC/NASTRAN offers two types of solution sequences for transient response analysis with time-dependent loads; the direct linear transient solution sequence (SOL109), and the direct nonlinear transient response solution sequence (SOL129). Since the aerodynamic loads acting on the blade are updated during the aeroelastic computation sequence and cannot be formulated as an explicit function of time, MSC/NASTRAN solves the structural response at each physical time step assuming constant loads during the period that starts with the initial conditions given by the solutions for the previous time step. SOL109 is typically used for models built with linear elements, but it cannot specify both initial displacements and velocities. Although the structural models used in this study are all built with linear elements, SOL109 is not properly suited for this particular application. Therefore SOL129 is used, because it can handle any initial condition as well as linear elements [82].

\subsubsection{Finite Element Model}

The finite element method offers many options for the structural dynamic analysis of swept and twisted blades. From these available options, plate or shell elements of rectangular or triangular shapes are commonly used since fan blades are quite thin [83]. In MSC/NASTRAN, several elements are available for shell structures. They can be both flat triangles (TRIA3, TRIA6), or flat rectangles (QUAD4, QUAD8). TRIA6 and QUAD8 are higher order elements that have quadratic interpolation functions; the displacements at any point in an element are assumed to be specified as quadratic functions of the nodal displacements. Meshes with higher order elements can, in theory, provide the same quality of results as meshes with with larger number of lower elements. However, any inherent error found in calculated results on a mesh 
consisting of lower order finite elements vanishes as the mesh is refined. From a practical standpoint, the blade, even if modeled with a relatively coarse lower order mesh should not result in large deviations from the exact solution. In this study, a simplified blade model that consists of varying-thickness linear shell elements is used.

TRIA3 and QUAD4 are the three-node and four-node triangular and quadrilateral linear finite elements provided by MCS/NASTRAN. These elements have six degrees of freedom per node, i.e., two membrane displacements, one membrane rotation, one bending displacement and two bending rotations. These six degrees of freedom can be easily converted into three translations and three rotations along and about the axes of a Cartesian coordinate system fixed in space. The element thickness is allowed to vary over the element surface. Lumped and consistent mass formulations are considered. As discussed later in Section 3.3, each fluid mesh point on the blade surface is associated with a projected point on the closest structural element surface for purpose of information exchange between the fluid and structural systems. Obviously, the search for projected points on a flat surface is much simpler than if the surface were curved. While the four nodes of a quadrilateral element do not always lie on a plane, the nodes of a triangular element always do independently of the element interpolation function. In this study, TRIA3 has been used because of its sufficient accuracy for the this type of fluid-structure interface.

\subsubsection{Damping Characteristics}

For turbomachinery blades, damping may be arising from aerodynamic forces, viscous damping, and material hysteresis [84]. For most blades the primary source of damping is aerodynamic damping due to the time-dependent aerodynamic loading generated by the blade motion. In an aeroelastic computation, the aerodynamic damping is calculated by the flow solver and is not included in the structural model. It depends on the rotation speed, pressure ratio, inter-blade phase angle, or any other 
factor that can affect the flow field. Unlike aerodynamic damping, the viscous and material damping characteristics are essentially determined by how the structure is built. Viscous damping gives rise to a force which opposes the motion proportionally to its velocity. It is mainly due to mechanical friction observed at the blade attachment to the disk or to the collision of the blade shrouds. It is considered to be very difficult to model viscous damping in structural models, in particular because the damping properties are usually frequency dependent. On the other hand, material damping is related to the total energy loss of the motion by material hysteresis which result in heat radiation. Although material damping is proportional to the square of frequency, this internal material damping is negligible even for the higher frequency modes, especially when the blade is made of a stiff material such as titanium. In this study, only viscous damping is included in the structural model for these reasons.

The viscous damping force is usually modeled as a function of a damping coefficient and the velocity of the structural body motion. It is represented in the equation of motion 3.46 using the damping matrix $[\boldsymbol{C}]$ and the velocity vector. Unlike mass and stiffness properties, damping properties are not easy to model in a general fashion. For this reason, damping models tend to be application specific. For instance, when the structural equations are reduced to the superposition of several dominant modes, the viscous damping coefficient is independently specified in the equation of motion for each mode. In usual aeroelastic analysis using finite element methods, the viscous damping is set to zero as its effects can be added later in a linear fashion. For example, the damping matrix $[\boldsymbol{C}]$ is explicitly constructed by assuming that it is a linear function of the mass matrix $[\boldsymbol{M}]$ and the stiffness matrix $[\boldsymbol{K}]$ of the complete element assembly, together with experimental results. For turbomachinery blades, it is more realistic to include the viscous damping by means of a stiffness proportional damping rather than a mass proportional damping. 
Incidentally, the material damping force depends on the displacements. It is expressed as a function of a damping coefficient and a complex component of the structural stiffness matrix. In practice, if the viscous damping force is assumed to be proportional to the stiffness force, it can be expressed by the same function. Let $G$ denote the viscous damping coefficient, then Equation 3.46 can be rewritten as

$$
[\boldsymbol{M}]\{\ddot{\boldsymbol{q}}\}+\{1+i G\}[\boldsymbol{K}]\{\boldsymbol{q}\}=\{\boldsymbol{f}\} .
$$

Consider the oscillatory response for a single degree of freedom system, $m \ddot{q}+c \dot{q}+k q=$ $f$, where $m, c, k, q$ and $f$ are the mass, the viscous damping coefficient, the stiffness coefficient, the displacement and the applied force, respectively. The solution of the equation of motion yields terms of type $q=q_{0} e^{-\zeta \omega t}$ which ensure that the time histories will decay for positive damping coefficients. The damping forces in Equation 3.48 and in the single degree of freedom system are identical if

$$
k G=c \omega .
$$

Therefore, if $G$ is to be modeled using an equivalent viscous damping $c$, then Equation 3.49 holds at only one frequency $\omega$. Two parameters $c$ and $\omega$ need to be specified to convert the viscous damping to an equivalent stiffness proportional damping. In this study, damping characteristics are modeled in this way choosing the first natural frequency of the structural model for $\omega$ and assuming $c$.

\subsubsection{Centrifugal and Coriolis Forces}

In predicting the natural modes and frequencies of rotor blades, it is essential to take into account the effect of the rotor rotational speed. The inertial forces due to the rotation of a blade tend to stiffen the transverse bending elastic springs. In vibration analysis, these forces approximately increase the square of the natural frequency $\omega_{n}^{2}$ 
in proportion to the square of the rotation speed $\Omega^{2}$. This effect can be described by stating $\omega_{n}^{2}=\omega_{0 n}^{2}+\kappa_{n} \Omega^{2}$ where $\omega_{0 n}$ is the non-rotating natural frequency of the rotor blade and $\kappa_{n}$ is a proportionality constant for the $n$th mode. The effect is most notable in the natural modes which exhibit predominantly bending displacements, usually the lowest frequency mode. Thus, for high-speed rotors, the inertial forces may be of importance in the aeroelastic problem.

These forces appear in the equations of motion as three additional terms. Assume that the rotating system spins about the $x$-axis with a constant frequency $\Omega$ relative to the stationary system, then the equations of motion for a particle in the rotating elastic structure with externally applied forces $\{\boldsymbol{f}\}$ can be written as,

$$
[\boldsymbol{M}]\{\ddot{\boldsymbol{q}}\}+\left([\boldsymbol{C}]-\Omega\left[\boldsymbol{C}^{c}\right]\right)\{\dot{\boldsymbol{q}}\}+\left([\boldsymbol{K}]-\Omega^{2}\left[\boldsymbol{K}^{c}\right]\right)\{\boldsymbol{q}\}=\{\boldsymbol{f}\}+\Omega^{2}\left[\boldsymbol{K}^{c}\right]\left\{\boldsymbol{q}_{0}\right\},
$$

where $\left\{\boldsymbol{q}_{0}\right\}$ is the position vector of the particle in the stationary system and $\{\boldsymbol{q}\}$ is the displacement of the particle. The term $\Omega\left[\boldsymbol{C}^{c}\right]\{\dot{\boldsymbol{q}}\}$ is commonly refered to as the Coriolis force and the term $\Omega^{2}\left[\boldsymbol{K}^{c}\right]\left(\left\{\boldsymbol{q}_{0}\right\}+\{\boldsymbol{q}\}\right)$ is refered to as the centrifugal force. Notice that the centrifugal force term in the right hand side of the equation behaves as a body force during the solution procedure. The matrices $\left[\boldsymbol{C}^{c}\right]$ and $\left[\boldsymbol{K}^{c}\right]$ for a system rotating about the $x$-axis can be written as,

$$
\left[\boldsymbol{C}^{c}\right]=\left[\begin{array}{ccc}
0 & 0 & 0 \\
0 & 0 & -2 m \\
0 & 2 m & 0
\end{array}\right], \quad\left[\boldsymbol{K}^{c}\right]=\left[\begin{array}{ccc}
0 & 0 & 0 \\
0 & m & 0 \\
0 & 0 & m
\end{array}\right]
$$

where $m$ is the mass of the particle. Notice that the centrifugal force is dependent on the current position vector of the particle, and divided into two terms: the term proportional to the stationary position vector and that proportional to the displacement vector. While the stiffness and damping terms in Equation 3.50 for a rotating 
system are the same as those in a stationary system, the part of the centrifugal term proportional to the displacement behaves as an additional stiffness term because it is proportional to the displacement. Similarly, the Coriolis term acts as additional damping in the equation of motion. These two terms are refered to as gyroscopic terms hereafter.

For the computation of frequencies and mode shapes using MSC/NASTRAN, it is possible to include the gyroscopic terms described above by using two solution sequences [85]. First, the gyroscopic terms are calculated in a steady-state displacement analysis run using solution sequence (SOL101) and the Direct Matrix Abstraction Program (DMAP) [86] included with the SSSAlter Library "segyroa.v705" [82]. The centrifugal force field in the right hand side of Equation 3.50 is computed by using the blade's stational geometry, mass properties and the rotational speed specified in the "RFORCE" card in the input file. This solution sequence computes steady-state displacements and then stores the blade modified stiffness and mass matrices in a database. Then, the gyroscopic terms are added to the global stiffness and damping matrices for the translational degree of freedoms in the direction of two axes perpendicular to the axis of rotation. Following SOL101, an eigenanalysis solution sequence (SOL103) is run to yield natural frequencies and mode shapes. Unfortunately, the current version of MCS/NASTRAN does not have the capability to include the gyroscopic terms in the direct transient response solution sequences which are required for solving the structural response of the blade with time-dependent nonlinear aerodynamic loads with initial conditions provided by the displacements and velocities of the last transient solution sequence.

Alternate approach taken in this study is to adjust the material properties of the rotating structure to create a stiffness model which accounts for gyroscopic effects. The gyroscopic effects are known to be significant on the first mode but are hardly recognizable on higher frequency modes for typical turbomachinery rotor blades [83]. 
An increase of the stiffness by changing material parameters such as Young's modulus, density and the thickness of the linear elements usually shifts all natural frequencies higher resulting in too high frequencies for higher frequency modes. However, the frequencies of higher frequency modes are not considered to influence the stability of the blade motions because flutter in turbomachinery is single degree of freedom instability as discussed in Chapter 1. Thus it is considered to be sufficient to model the structure with a realistic lowest frequency for observing the aeroelastic behavior of the blade in a flutter condition.

\subsubsection{Time Integration}

Efficient and accurate numerical integration schemes for a second order system, such as Equation 3.46, have been proposed by many researchers [80]. Houbolt presented an implicit, three-step recurrence scheme. The most popular Newmark method provides a single-step family of methods employing two integration parameters $\left(\beta_{N}, \gamma_{N}\right)$ to give a variety average approximation of the acceleration term. Wilson introduced a family of implicit methods employing a single integration parameter. Katona and Zienkiewicz [87] introduced a generalization of Newmark's time marching integration scheme, called beta- $m$ method, in which the most well-known methods (e.g. Newmark, Wilson, Houbolt, etc.) are shown to be special cases within the beta-m family. In the present work, the equations of structural motion are advanced in time by using a modified Newmark method, which is unconditionally stable, included in MSC/NASTRAN [88].

When the displacements are solved for at discrete times, typically a fixed integration time step $\Delta t$ is used. A central finite difference representation of the velocity $\{\dot{\boldsymbol{q}}\}$ and acceleration $\{\ddot{\boldsymbol{q}}\}$ vectors at discrete times becomes, 


$$
\begin{aligned}
& \left\{\dot{\boldsymbol{q}}_{n}\right\}=\frac{1}{2 \Delta t}\left(\left\{\boldsymbol{q}_{n+1}\right\}-\left\{\boldsymbol{q}_{n-1}\right\}\right), \\
& \left\{\ddot{\boldsymbol{q}}_{n}\right\}=\frac{1}{\Delta t^{2}}\left(\left\{\boldsymbol{q}_{n+1}\right\}-2\left\{\boldsymbol{q}_{n}\right\}+\left\{\boldsymbol{q}_{n-1}\right\}\right) .
\end{aligned}
$$

Averaging the displacement and the applied force over three adjacent time steps, the structural equations of motion 3.46 can be rewritten as,

$$
\begin{array}{r}
{\left[\frac{M}{\Delta t^{2}}\right]\left(\left\{\boldsymbol{q}_{n+1}\right\}-2\left\{\boldsymbol{q}_{n}\right\}+\left\{\boldsymbol{q}_{n-1}\right\}\right)+\left[\frac{C}{2 \Delta t}\right]\left(\left\{\boldsymbol{q}_{n+1}\right\}-\left\{\boldsymbol{q}_{n-1}\right\}\right)} \\
+\left[\frac{K}{3}\right]\left(\left\{\boldsymbol{q}_{n+1}\right\}+\left\{\boldsymbol{q}_{n}\right\}+\left\{\boldsymbol{q}_{n-1}\right\}\right)=\frac{1}{3}\left(\left\{\boldsymbol{f}_{n+1}\right\}+\left\{\boldsymbol{f}_{n}\right\}+\left\{\boldsymbol{f}_{n-1}\right\}\right) .
\end{array}
$$

Rearranging the equation, it becomes

$$
\left[A_{1}\right]\left\{\boldsymbol{q}_{n+1}\right\}=\left\{\boldsymbol{f}_{1}\right\}+\left[A_{2}\right]\left\{\boldsymbol{q}_{n}\right\}+\left[A_{3}\right]\left\{\boldsymbol{q}_{n-1}\right\},
$$

where,

$$
\begin{aligned}
{\left[A_{1}\right] } & =\left[\frac{M}{\Delta t^{2}}+\frac{1}{2 \Delta t} C+\frac{K}{3}\right], \\
{\left[A_{2}\right] } & =\left[\frac{2 M}{\Delta t^{2}}-\frac{K}{3}\right], \\
{\left[A_{3}\right] } & =\left[-\frac{M}{\Delta t^{2}}+\frac{C}{2 \Delta t}-\frac{K}{3}\right], \\
\left\{\boldsymbol{f}_{1}\right\} & =\frac{1}{3}\left(\left\{\boldsymbol{f}_{n+1}\right\}+\left\{\boldsymbol{f}_{n}\right\}+\left\{\boldsymbol{f}_{n-1}\right\}\right) .
\end{aligned}
$$

This approach is similar to the classical Newmark direct integration method except that $\boldsymbol{f}$ is averaged over three time points. The transient solution is obtained as a series of static solutions at each time step by factoring $\left[A_{1}\right]$ and applying it to the righthand side of the above equation which consists of all the known variables. Assuming 
that, the $[M],[C]$, and $[K]$ matrices are constant throughout the analysis and do not change with time, the factorization of $\left[A_{1}\right]$ can be stored and does not need to be recomputed if the time step, $\Delta t$, is constant. Under similar considerations, $\left[A_{2}\right]$ and $\left[A_{3}\right]$ also remain constant throughout the analysis. The transient nature of the solution is carried through by modifying the applied force vector, $\boldsymbol{f}_{1}$.

\subsection{Fluid-Structure Interface}

As discussed at the beginning of this chapter, the flow and structural solvers used in this study have been independently developed and an appropriate interface needs to be developed to couple the two solvers. In early fluid/structure coupled aeroelastic calculations for external flows, the same surface discretization for the fluid and structural domains with the same size of the surface elements was used to transfer loads and deformations between the two disciplines without the need for any interpolations [89]. The same assumption was made for turbomachinery flow by Imregun and his collaborators $[52,53]$. The fluid system and the structural systems, however, essentially use different surface discretizations because of differing accuracy requirements in the resolution of the features of each solution. The fluid system usually requires a finer mesh to capture nonlinear features such as shock waves and boundary layers. Moreover, the type of element that each solver uses might also cause mismatches of the mesh points on the interface. In this study, while the flow solver employs relatively finer multiblock structured meshes, the structural solver employs triangular elements. This mismatch of mesh points on the interface gives rise to the necessity of a numerical approximation to transfer the pressure distribution on the fluid surface mesh to a corresponding nodal load distribution on the structure and also for the transfer of the displacements of the structural nodes to corresponding perturbations of the fluid mesh. 
The level of the accuracy in the coupling process must be carefully considered so as not to forego solution quality sought by the high accuracy in the discretization of the individual disciplines. One of the principles that is needed to support the maintenance of coupling accuracy is the conservation of loads and energy given in Section 2.3.1 which states that the sum of the nodal forces in the structural system transfered from the pressure field in the fluid system must be equal to the integral of the pressure fields on the body surface boundary in the fluid system. In this study, the approach formulated from the conservation of loads and energy by Brown [90], and later followed by Reuther et al [91] in aero-structural optimization, is chosen to ensure that the transfer of the pressure fields to the nodal forces is both consistent and conservative. Brown's approach can also be used in the extrapolation of the nodal displacement in the structural system to the mesh deformation on the surface of the fluid system.

In addition, the fluid system usually requires a finer temporal resolution than the structural vibration for most aeroelastic problems. In computations, while the flow solver employs the Runge-Kutta time integration which has a stability limit, the structural solver uses the Newmark time integration which is unconditionally stable. This difference in the stability limits need to be taken care of by a synchronization approach between the two solvers alternatively advancing in time with different time steps.

In the following sections, the detail descriptions are given for the individual interface algorithm starting from the deformation tracking system, followed by the load transfer system and the synchronization.

\subsubsection{Deformation Tracking System}

In most cases of aeroelastic analysis of turbomachinery, the blade may be simply modeled with shell elements which usually have fewer nodal points than the outer 
mold surface defined by the fluid mesh. Since it is modeled with shell elements, every fluid mesh point on the blade surface, which is on the outer mold surface, can be said to exist outside of the finite element structural model. In finite element analysis, the displacement at any point within the domain of an element in the model can be determined by the assumed finite element interpolation functions in terms of the nodal displacements on the element. Therefore, if a fluid mesh point on the outer mold surface can be associated with a point on a structural element in a certain way, the deformed location of the point on the outer mold surface may be calculated as a function of the nodal displacements on the structural element.

In developing these extrapolation functions, first each individual point on a fluid mesh must be tied to the nearest point on the underlying finite element model surface before any deformation takes place. Consider a set of finite elements describing a structural model and a fluid mesh point on the outer mold surface shown in Figure 3.10. The structural model consists of shell elements which have three nodes and eighteen degrees of freedom. Here the fluid mesh point is located on the actual blade surface. This simply means that the fluid mesh point will never lie on the surface of the shell element as long as the blade has finite thickness. Suppose that the translational and rotational displacements at a point $\boldsymbol{X}$ on the fluid mesh surface are denoted by $\boldsymbol{\mu}(\boldsymbol{X})$ and $\boldsymbol{\mu}_{\theta}(\boldsymbol{X})$ respectively, it can be assumed that a normal vector which is perpendicular to the closest shell element from $\boldsymbol{X}$ before deformation remain perpendicular after deformation. Thus, each normal vector can be assumed to move as a rigid body. Let $\boldsymbol{x}$ denote the closest point on the closest element from $\boldsymbol{X}$, the displacements $\boldsymbol{\mu}(\boldsymbol{X})$ and rotations $\boldsymbol{\mu}_{\theta}(\boldsymbol{X})$ can be expressed as follows,

$$
\begin{aligned}
\boldsymbol{\mu}(\boldsymbol{X}) & =\boldsymbol{u}(\boldsymbol{x})-(\boldsymbol{X}-\boldsymbol{x}) \times \boldsymbol{u}_{\theta}(\boldsymbol{x}) \\
\boldsymbol{\mu}_{\theta}(\boldsymbol{X}) & =\boldsymbol{u}_{\theta}(\boldsymbol{x})
\end{aligned}
$$



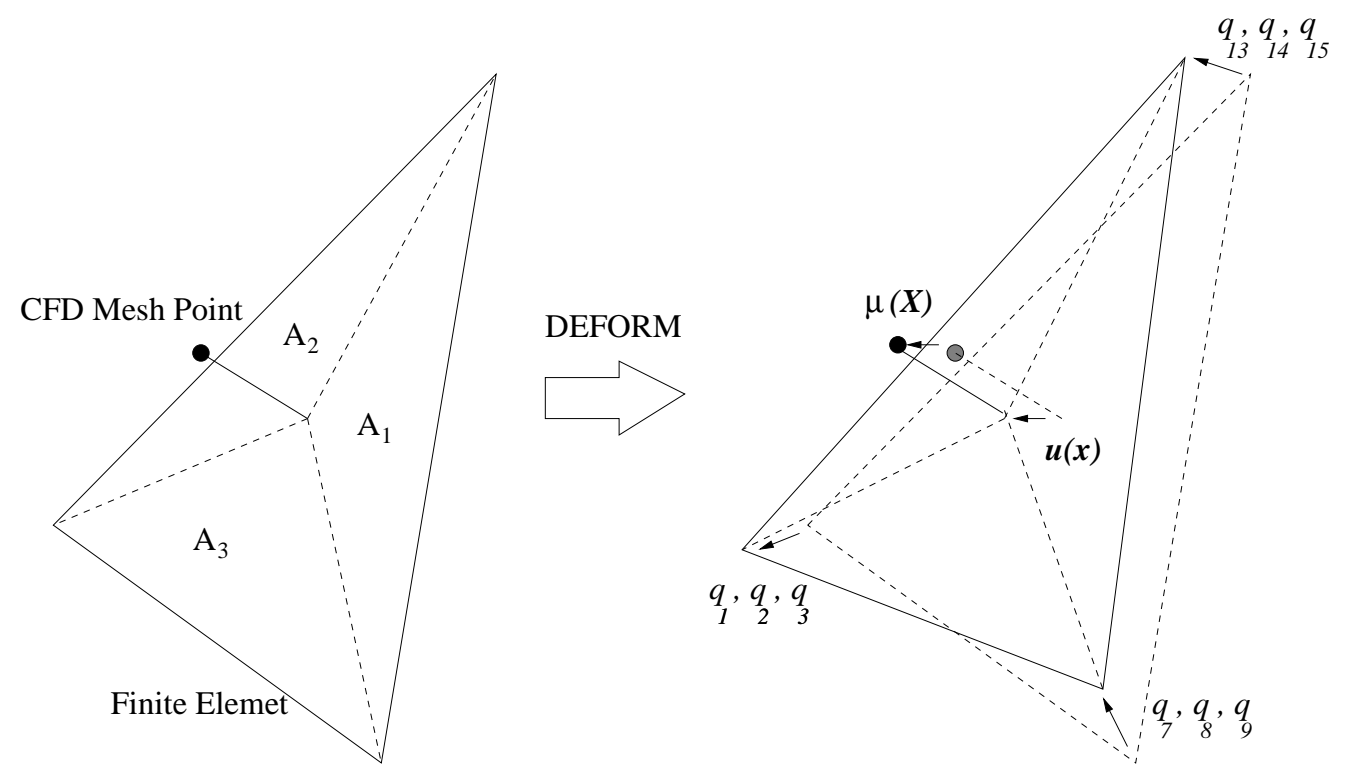

Figure 3.10: Deformation Tracking System

where $\boldsymbol{u}(\boldsymbol{x})$ and $\boldsymbol{u}_{\theta}(\boldsymbol{x})$ are the displacement and rotation at $\boldsymbol{x}$. Given a set of values for the eighteen nodal displacements, displacements and rotations at any point on a triangle shell element can be evaluated using the original finite element interpolation functions. However, since MSC/NASTRAN is used as a structural solver and the finite element interpolation function used in it is unknown, all of the finite elements were assumed to use the simple standard iso-parametric interpolation function based on the area coordinate as the weighting. When the fluid mesh point $\boldsymbol{X}$ is associated with $\boldsymbol{x}$ on the $m$ th element whose displacements are denoted as $\boldsymbol{q}^{(m)}$, this interpolation function can be expressed as,

$$
\begin{aligned}
\boldsymbol{u}(\boldsymbol{x}) & =\left[\eta^{(m)}(\boldsymbol{x})\right] \cdot \boldsymbol{q}^{(m)}=\left[\left[A_{1}^{(m)}\right][0]\left[A_{2}^{(m)}\right][0]\left[A_{3}^{(m)}\right][0]\right] \cdot \boldsymbol{q}^{(m)}, \\
\boldsymbol{u}_{\theta}(\boldsymbol{x}) & =\left[\eta_{\theta}^{(m)}(\boldsymbol{x})\right] \cdot \boldsymbol{q}^{(m)}=\left[[0]\left[A_{1}^{(m)}\right][0]\left[A_{2}^{(m)}\right][0]\left[A_{3}^{(m)}\right]\right] \cdot \boldsymbol{q}^{(m)},
\end{aligned}
$$

where $[0]$ is the $3 \times 3$ zero matrix and $\left[A_{1}^{(m)}\right],\left[A_{2}^{(m)}\right]$ and $\left[A_{3}^{(m)}\right]$ are defined by the area 
coordinates $[80]$. For example, $\left[A_{1}^{(m)}\right]$ is expressed using the area coordinate shown in Figure 3.10 as follows,

$$
\left[A_{1}^{(m)}\right]=\left[\begin{array}{ccc}
A_{1} & 0 & 0 \\
0 & A_{1} & 0 \\
0 & 0 & A_{1}
\end{array}\right]
$$

The displacements and rotations at any point on the fluid mesh surface can thus be written in terms of the associated nodal displacements $\boldsymbol{q}^{(m)}$ using Equation 3.57.

$$
\begin{aligned}
\boldsymbol{\mu}(\boldsymbol{X}) & =\left[\eta^{(m)}(\boldsymbol{x})\right] \cdot \boldsymbol{q}^{(m)}-[\boldsymbol{X}-\boldsymbol{x}] \cdot\left[\eta_{\theta}^{(m)}\left(\boldsymbol{x}_{n}\right)\right] \cdot \boldsymbol{q}^{(m)} \\
\boldsymbol{\mu}_{\theta}(\boldsymbol{X}) & =\left[\eta_{\theta}^{(m)}(\boldsymbol{x})\right] \cdot \boldsymbol{q}^{(m)}
\end{aligned}
$$

where, the matrix $[\boldsymbol{X}-\boldsymbol{x}]$ in cross product form can be given by,

$$
[\boldsymbol{X}-\boldsymbol{x}]=\left[\begin{array}{ccc}
0 & (z-Z) & (Y-y) \\
(Z-z) & 0 & (x-X) \\
(y-Y) & (X-x) & 0
\end{array}\right]
$$

Since the rotations of the fluid mesh points are not used in the actual computations for the flow filed, only $\boldsymbol{\mu}(\boldsymbol{X})$ needs to be calculated. Introducing the displacement extrapolation functions $N(\boldsymbol{X})$ based on the global nodal displacements $\boldsymbol{q}$ of the structural model, Equation 3.59 can be rewritten using $\boldsymbol{q}$ because $\boldsymbol{q}^{(m)}$ is a part of $\boldsymbol{q}$.

$$
\boldsymbol{\mu}(\boldsymbol{X})=[N(\boldsymbol{X})] \cdot \boldsymbol{q}
$$

where,

$$
[N(\boldsymbol{X})]=[\eta(\boldsymbol{x})]-[\boldsymbol{X}-\boldsymbol{x}] \cdot\left[\eta_{\theta}(\boldsymbol{x})\right]
$$

As the interpolation functions $\eta(\boldsymbol{x})$ and $\eta_{\theta}(\boldsymbol{x})$ linearly evaluate the displacements 
within the element, $N(\boldsymbol{X})$ can be used to extrapolate the displacements linearly to the point outside of the element. As long as the displacements are small enough for the structure to be modeled with linear elements, these extrapolation functions are accurate, and the simplest method satisfying a condition that the displacements $\boldsymbol{\mu}(\boldsymbol{X})$ must be continuous in the region of fluid mesh. The procedure to obtain these interpolation functions $N(\boldsymbol{X})$ is implemented in a preprocessing program followed by the main solver. $N(\boldsymbol{X})$ is thus precomputed and stored for later use during the aeroelastic procedure and plays an important role in the load transfer algorithm as discussed in the next section.

The computational approach for mesh perturbation described in Section 3.1.8 will then move the mesh point in the whole fluid computational domain according to the explicit perturbation of the fluid mesh points on the fluid/structure interface. In the dual time stepping scheme, mesh velocities are necessary as well as the mesh position. To obtain the mesh velocities, a backward difference is used with the old mesh positions which are stored in the memory. No extrapolation from the nodal velocity of the structural model is used even on the interface.

\subsubsection{Load Transfer System}

In this section, a method that transfers aerodynamic loads obtained through a stress integration over the structural surface area in the fluid system to applied nodal forces with which the structural equations of motion must be solved is described. In general, aerodynamic forces that act on a structural surface are divided into two categories except for the body forces already ignored in the fluid equations. One is pressure forces pushing the surface perpendicularly to the surface, the other is viscous shear forces which appear when viscous calculations are performed in the fluid system. Since the magnitude of shear forces is trivial compared to that of pressure forces, viscous shear forces are not transfered from the fluid system to the structural system 
in this study.

For a load transfer algorithm, it is required to satisfy the conservation of load and energy expressed in Equations 2.36 and 2.37, respectively. In Equation 2.37, $\delta \boldsymbol{\mu}(\boldsymbol{X})$ can be related with $\delta \boldsymbol{q}$ introducing a set of assumed displacement interpolation functions $N(\boldsymbol{X})$ given in Equation 3.61. Substituting Equation 3.61 into Equation 2.37 yields,

$$
\boldsymbol{f} \cdot \delta \boldsymbol{q}=\int_{\partial \Omega} p \cdot[N(\boldsymbol{X})] \delta \boldsymbol{q} d \boldsymbol{S},
$$

where $\boldsymbol{f}$ is the global nodal force vector for the structural model. In practice, the fluid pressure is taken as constant over a face around a fluid mesh point. Consider the complementary projected surface area vector $\boldsymbol{S}_{i}$ around the fluid mesh point $\boldsymbol{X}_{i}$ surrounded by the corner points which are given by simply averaging the corner points of each face of the fluid mesh as shown in Figure 3.11, the right hand side of the Equation 3.63 can be discretized as follows,

$$
\boldsymbol{f} \cdot \delta \boldsymbol{q}=\sum_{i} p_{i} \boldsymbol{S}_{i} \cdot\left[N\left(\boldsymbol{X}_{i}\right)\right] \delta \boldsymbol{q} .
$$

This allows the fluid pressure within each face to be taken out of the summation,

$$
\boldsymbol{f}_{i}=\left\{p_{i} \boldsymbol{S}_{i}\right\}\left[N\left(\boldsymbol{X}_{i}\right)\right],
$$

where $\boldsymbol{f}_{i}$ is the nodal force vector given by the contribution of the pressure at the fluid mesh point $\boldsymbol{X}_{i}$, which components are shown in Figure 3.11. The nodal force vector for the $m$-th element denoted by $\boldsymbol{f}^{(m)}$ is now obtained by simply summing the all $\boldsymbol{f}_{i}$ whose fluid mesh point is associated with the $m$-th element.

At this point, the algorithm ensures that it satisfies the conservation of energy on which the algorithm is based. The next question is whether it satisfies the conservation of loads given in Equation 2.36. Since the interpolation function $\left[N\left(\boldsymbol{X}_{i}\right)\right]$ consists of 


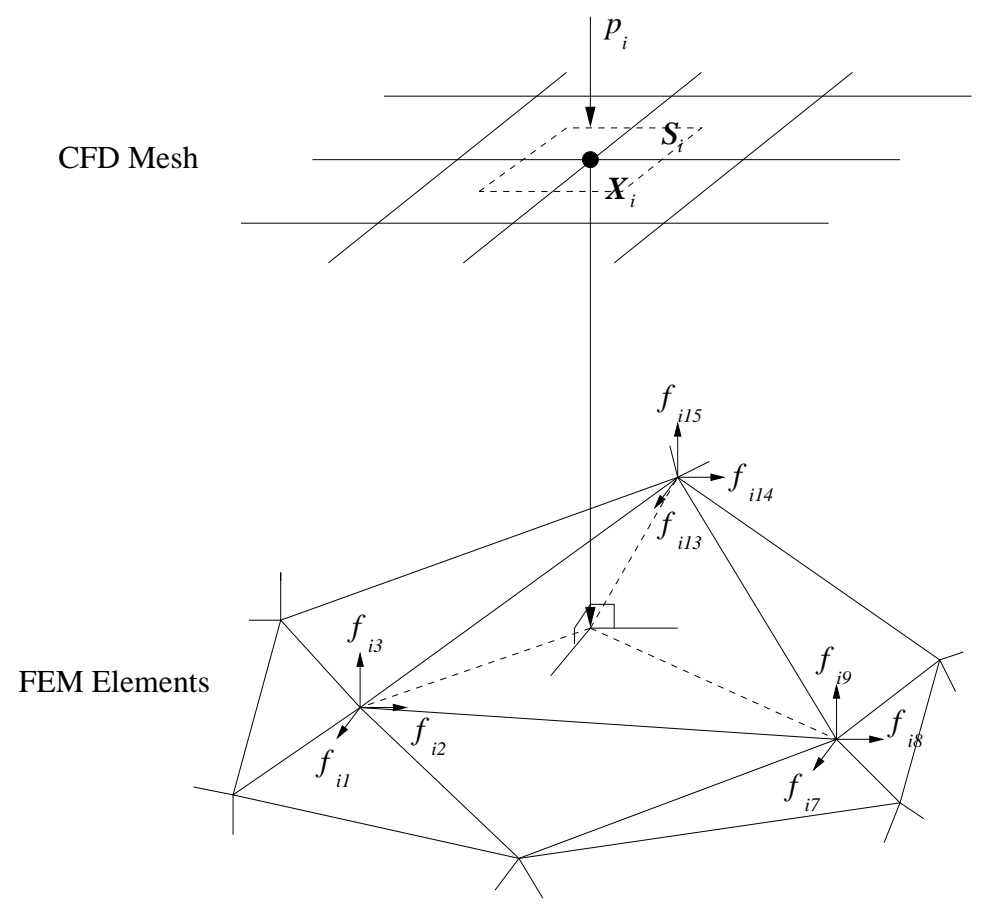

Figure 3.11: Load Transfer System

the area coordinate system normalized by the entire area of the element associated with the point $i$, it is obvious that the norm of the function is equal to one. Therefore, the conservation of loads is easily validated by collecting $\boldsymbol{f}_{i}$.

$$
\sum_{m} \boldsymbol{f}^{(m)}=\sum_{i} \boldsymbol{f}_{i}=\sum_{i}\left\{p_{i} \boldsymbol{S}_{i}\right\}\left[N\left(\boldsymbol{X}_{i}\right)\right]=\sum_{i}\left\{p_{i} \boldsymbol{S}_{i}\right\}=\int_{\partial \Omega} p d \boldsymbol{S} .
$$

Thus the algorithm satisfies the conservation of loads and energy.

\subsubsection{Synchronization}

As described above, the fluid and structure solvers have been developed with different numerical methods. Consequently, the simultaneous solution of both disciplines by a monolithic scheme is in general computationally challenging. Alternatively, both 
calculations are performed separately and synchronized. In many aeroelastic calculations, a steady flow is first computed around a structure in equilibrium or in a given stationary condition with a set of initial displacements. Next the structure is allowed to respond to the aerodynamic forces determined by the steady flow field. This should be followed by a simple and popular staggered procedure as described below.

(1) Transfer the motion of the boundary of the structure to the fluid system using the Deformation Tracking System and update the fluid mesh accordingly. At this time, the updated cell volumes, projected areas and surface normals of all the cell faces are calculated.

(2) Advance the fluid system $\boldsymbol{W}^{n+1}$ to a steady-state in pseudo-time and obtain new pressure fields using the solutions at the time levels $n, n-1$, and $n-2$ stored in memory for the third order accurate backward time discretization. An initial guess for the solution at time level $n+1$ is made equal to the solution at the previous time level.

(3) Convert the new pressure fields into a set of nodal forces for the structural model using the Load Transfer System.

(4) Advance the structural system under the given set of nodal forces.

The procedure repeats from step (1) through (4) until the desired time-marching solutions are obtained. The basic staggered algorithm outlined above is graphically illustrated in the left diagram of Figure 3.12 where $\boldsymbol{W}$ denotes the flow variables, $\boldsymbol{q}$ denotes the structural displacements, and $p$ denotes the fluid pressure. The superscript $n$ designates the $n$-th time-station. The simplicity of this procedure is attractive and apparently has earned the highest popularity among synchronization procedures for aeroelastic computations in the time domain.

In most of the aeroelastic problems, different time scales are chosen for the fluid and structure systems. The fluid system usually requires a finer time resolution than the structural system. For the flow solver, an explicit scheme such as Runge-Kutta 


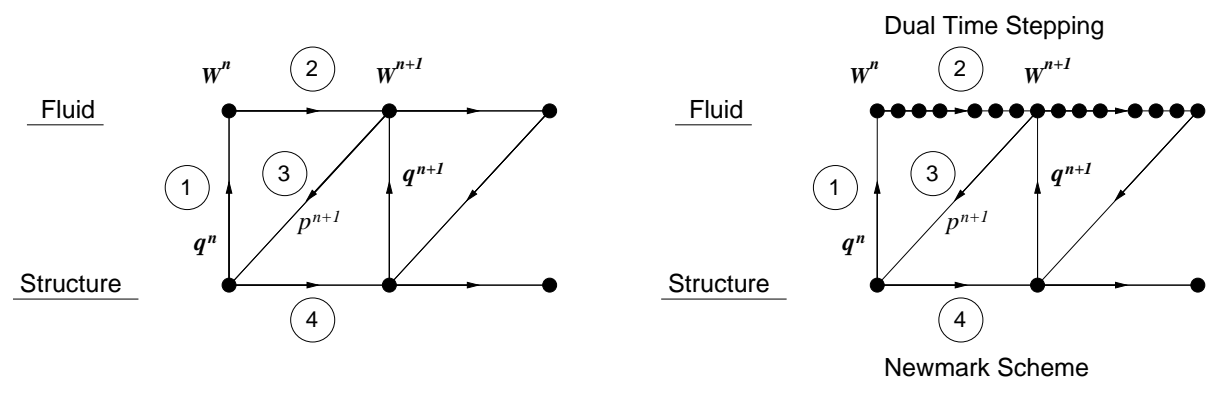

Figure 3.12: Simple Partitioned Stagger Procedures

scheme must be selected to integrate the semi-discrete equations in order to obtain the time accurate solutions for each period. On the other hand, the implicit Newmark trapezoidal rule, which is unconditionally stable, is usually selected to integrate the equation of motion of the structure. If the procedure mentioned above is used to solve the aeroelastic equations, the time step must be governed only by the critical time step of the explicit fluid solver. It is also noted that since the fluid system must be advanced in the physical time scale, the fast convergence methods such as the local time stepping, or multigrid can not be available. Thus, using the same time step in both fluid and structure system is not efficient in advancing the structural system. This difference in allowable time step size is, however, overcome by the dual time stepping scheme in which the fluid system is updated implicitly in physical time, and the pseudo time step is introduced to integrate the fluid equations within the physical time period as discussed in Section 3.1.3. In this way, the same physical time step is used for both solvers [53], because the physical time step of the dual time stepping in fluid calculation can be chosen arbitrary and the structural time step has no limitation. Thus, the overall computational cost can be saved because fewer computations are necessary for the structural solver and communication in exchanging boundary information. The basic assumption for this method is that during one time-step of the fluid, structural deflections are small, and that during 
one time-step of the structure, the flow field (loads) remain approximately constant. This procedure is also graphically illustrated in the right diagram of Figure 3.12.

As far as the accuracy in time is concerned, the dual time stepping scheme is at least second-order accurate in time. The trapezoidal rule is also second-order accurate. The resulting simple partitioned staggered procedure becomes first-order accurate in time according to a simple Taylor expansion [62]. This particular choice of the mixed time integration is motivated by the fact that the aeroelastic response of a structure is often dominated by low frequency modes and therefore is most efficiently predicted by an implicit time integration scheme.

\subsection{Parallelization}

A fluid/structure coupled time-marching computation is computationally very expensive especially applied to a viscous flow. The cost may became particularly extravagant when it is used to detect flutter boundaries for turbomachinery because many cases may need to be performed changing conditions such as rotation speeds, pressure ratios, and inter-blade phase angles. Parallel computing is one possibility to reduce the computational cost for multidisciplinary computations so that these computations could be of more practical use for the preliminary design process of turbomachinery.

As discussed in Section 3.3.3, the basic procedure for the coupling of the two disciplines is to advance both systems alternatively in time. Farhat [62, 92] also addressed a possibility of advancing the fluid and structure equations in parallel. In this method, the fluid and structure systems can run in parallel during the time interval independently in different processors and exchange information on the interface at the end of each period. However, the fluid solver usually requires much more computational cost than the structure solver. As a result, the processors in charge of the structure part 
may need to wait for a long time until the flow calculations are finished to be ready to exchange information for the next time step. Given a number of processors, the parallelization between the fluid and structure solvers might not help reduce the total aeroelastic simulation time. Therefore, the parallelization strategies for this study are considered independently on the fluid and structure solvers and more effort must be made for the fluid solver than the structural solver.

For the flow solver, the multiblock method is conveniently used in the finite volume discretization of the flow filed over complex geometries. This method is based on domain decomposition technique, with the flow domain being partitioned into a number of sub-domains. Within each sub-domain, the flow equations are solved in an independent manner, with the global nature of the flow being accounted for by the periodic exchange of boundary information between neighboring blocks. This domain decomposition is designed not only for representing the complex geometry but also helping a parallelization strategy [93]. The parallelization strategy adapted in TFLO is based on this multiblock decomposition discussed in detail in this section. This section also discussed on the parallelization of the structural solver which is very simple and more intended for memory reduction rather than fast computation.

\subsubsection{Flow Solver}

In the multiblock strategy, the space in the computational domain surrounding a geometry of interest is filled with a series of structured blocks of varying sizes. As mentioned in Section 3.1.6, each block is surrounded by halo cells in which proper boundary conditions are applied. The multiblock strategy makes use of these halo cells to communicate with the neighboring blocks. By updating the flow variables in the halo cells with those of the corresponding interior cells in the neighboring blocks, the multiblock flow solver is allowed to proceed independently within each block during each stage of the Runge-Kutta time integration procedure. This inter-block 
communication for updating the variables in the halo cells is performed at the end of each stage of the Runge-Kutta scheme at each level of the multigrid when the solver calls the boundary conditions.

When the flow filed is partitioned into blocks and the number of processors used for the computation is specified, the computational load for each processor can be evenly distributed. According to the multiblock decomposition, the even distribution of the computational loads is attempted by dividing the blocks into the same number of groups as the number of processors used in the computation such that the differences among processors in terms of the amount of computation and communication that they must perform is a minimum [93]. The number of blocks assigned to each processor is not necessarily identical because the size of the block is usually not equal. Typically, each processor is assigned a set of blocks of varying sizes but approximately an equal total number of cells. In addition, the communication cost is also taken care of by attempting to place blocks that share a physical interface on the same processors such that as much inter-block communication is processed within each processor as possible to minimize the inter-block communication between different processors.

The data communication between fluid blocks assigned to different processors is accomplished by using the Message Passage Interface (MPI) standard (wwwunix.mcs.anl.gov/mpi/index.html), which is a set of libraries for message passing within a multi-processor computer. For example, MPI subroutines are called when the solver applies boundary conditions at every stage of the Runge-Kutta time integration, or perturbs corner points and edges implicitly affected by the blade motion, etc. Each processor individually proceeds to the computation of the next time step as soon as its inter-block communication phase is completed not holding until all the inter-block communications are done.

In the implementation of the parallelization scheme using the multiblock strategy, a pre-processing module identifies the halo cells adjacent to the block boundaries and 
constructs a list for each processor which contains the pointers for the halo cells in the neighboring blocks and the corresponding processor number. The pre-processor also calculates the computational and communication load for each block and performs the load balancing for the parallel computation at the same time. Based on the information on the list for each processors, the excursion of different programs onto different processor are enabled by using an MPI command.

\subsubsection{Structural Solver}

MSC/NASTRAN has the capability of parallel processing in its package. This parallelization is intended to decompose the global matrices into smaller ones and solve partitioned nodal displacements for a group of degree of freedoms in parallel, especially when the structure is modeled with a massive number of elements. However, since the size of each blade structural model is not so large as to have a serious influence on the aeroelastic computational cost, the parallelization strategy for the structural solver used in this study should rather be directed at the multi-passage computations in which multiple structural models of the blades need to be analyzed at the same time. From the numerical experiment, MSC/NASTRAN can be executed from different processors in a parallel computer with different input files at the same time, and it yields the different results.

In multi-passage calculations, each blade has its own unique structural model. The structural models are allocated in the processors so that the number of structural models per processor is minimized. The number of structural models is usually less than the number of blocks for the fluid calculations, and also less than the number of processors used in the parallel computations. It is simply considered that some of the processors possess the structural models, run the structural solver and distribute the resulting solutions to the corresponding processors using MPI. 


\section{Chapter 4}

\section{Results}

\subsection{Code Evaluation - Cantilever Double Circular Arc Airfoil Wing}

In order to evaluate the accuracy of flutter boundary predictions, the fluid/structure coupled solver was compared with experimental measurements and other aeroelastic predictions for a transonic unswept rectangular cantilever double circular arc airfoil wing. For this wing, Doggett et al [94] conducted an experimental investigation on flutter speeds at various Mach numbers, and Gurusmamy [89] and Alonso [95] performed fluid-structure coupled computations with an Euler flow solver and a superposition of the response for a couple of the lowest frequency modes of the structure. The current computations are also performed with an Euler solver.

A diagram of this wing is shown in Figure 4.1. The length and the width of the model are respectively 4.56 and 11.50 inches. The aspect ratio is 5.0. Each airfoil section consists of a 6 percent thick double circular arc airfoil. The experimental model was constructed of a 0.065 inch thick aluminum-alloy flat-plate insert and a light weight covering which formed the airfoil cross-section. Thus, the model was designed such that the structural properties were concentrated in the aluminum insert. 


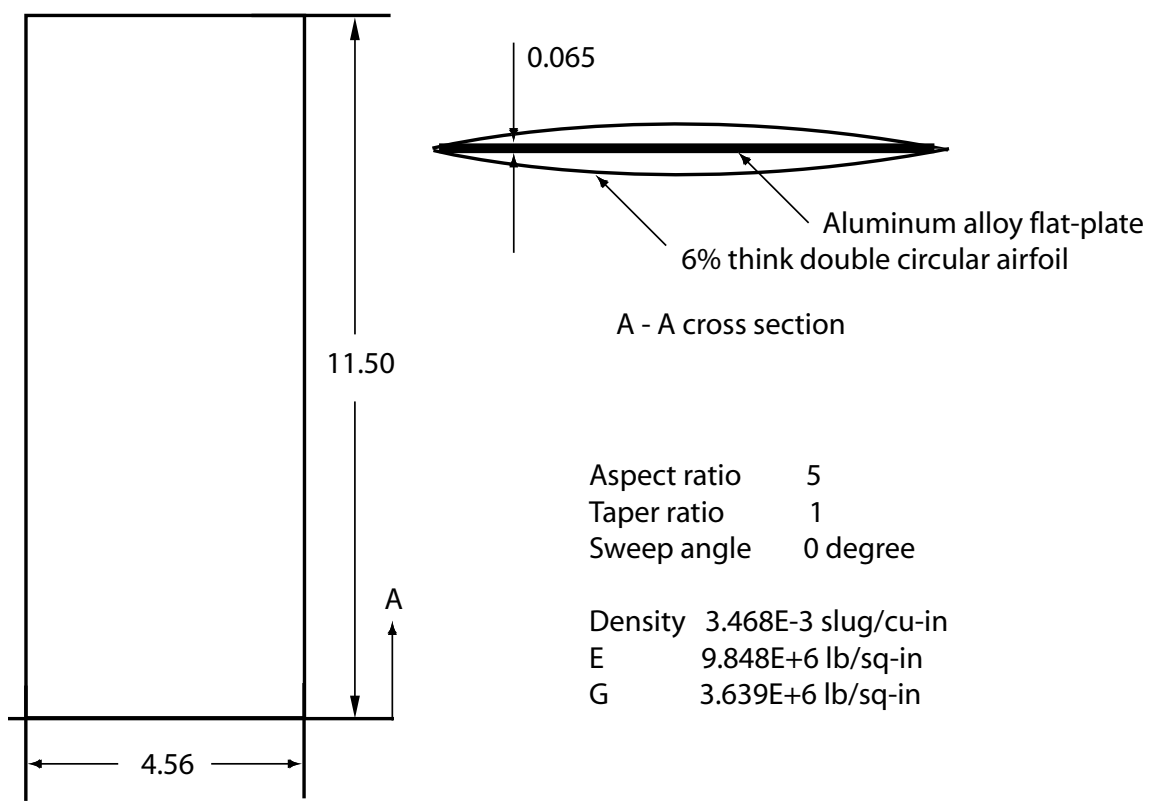

Figure 4.1: Cantilever Double Circular Airfoil Wing

The measured structural properties of the model are also presented in Figure 4.1.

The aeroelastic responses are computed by modeling the wing with three-nodes triangular plate elements called TRIA3 in NASTRAN that has three in-plane degrees of freedom and three bending degrees of freedom at each node. It has 18 degrees of freedom in total. Since the influence of the light weight covering is considered to be negligible, the structural model for this wing is simulated only for the aluminumalloy flat-plate, and the covering is not included. The structural model consists of 200 elements in an $11 \times 11$ nodes mesh. Viscous damping is not included in this model. The modal shapes and natural frequencies of the wing were obtained from the finite element eigenproblem of the structure solved by NASTRAN. The first four normal modes are shown in Figure 4.2. The mode shapes are shown with the deformed shape of the surface mesh around the wing. These mode shapes and frequencies compare quite well with the measured data for the experimental model. The small difference in those values is considered to be due to the presence of the light weight covering 


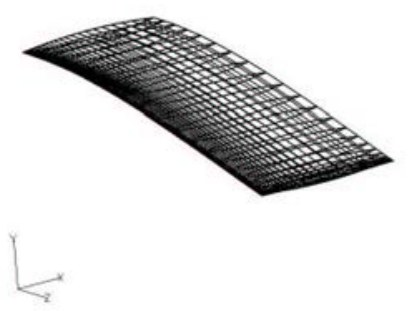

1st Bending Mode

NASTRAN - $14.85 \mathrm{~Hz}$

Measurement $-14.29 \mathrm{~Hz}$

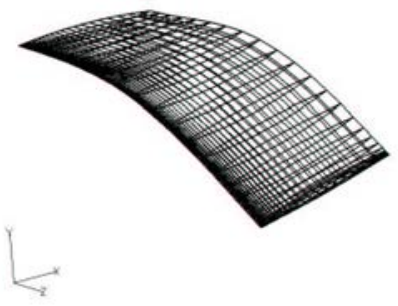

2nd Bending Mode

NASTRAN - $91.62 \mathrm{~Hz}$

Measurement - $89.80 \mathrm{~Hz}$

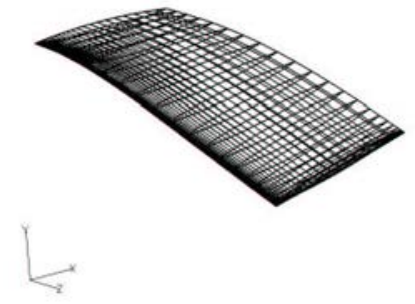

1st Torsion Mode

NASTRAN - $75.25 \mathrm{~Hz}$

Measurement $-81.41 \mathrm{~Hz}$

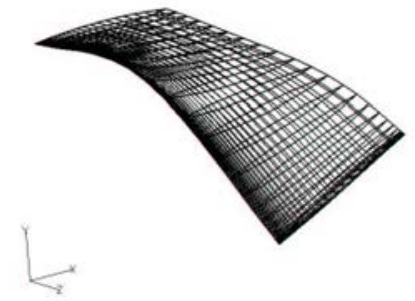

2nd Torsion Mode NASTRAN - $239.43 \mathrm{~Hz}$

Figure 4.2: Mode Shapes of the Cantilever Double Circular Arc Airfoil Wing

which is not accounted for in the structural model.

In the aerodynamic part of the calculation, an H-type mesh shown in Figure 4.3 was used. There were 73 points in the stream-wise direction, 41 points in the span-wise direction and 33 points in the normal direction. There are 25 points in the chord-wise direction between the leading and trailing edges and 25 points in the spanwise direction between the wing root and tip on both the upper and lower surfaces of the wing. The aeroelastic computations were initiated with the converged steadystate flow solutions for the wing with initial modal disturbances in the first mode. The maximum displacement of the initial disturbances on the blade, absolutely appears at 
a point on the tip, was set to be 0.8 inch. Once the initial steady-state was obtained, the wing was released from its forced deflection state and allowed to move. The structural motion of the wing and the flow around the wing are periodically updated to give a time history of any quantity that represents the flow field and the structural motion. They fluid/structure coupled solutions computed over a range of freestream Mach numbers from $M_{\infty}=0.7$ to $M_{\infty}=1.0$ for various freestream dynamic pressures. In the current investigation, the calculation is repeated by holding Mach number and all other parameters constant except for the freestream dynamic pressures and increasing the freestream dynamic pressure gradually until a flow condition is found for which a constant amplitude motion is obtained that determines the flutter point. In general, the growth or decay of disturbances of an isolated wing has been found to be dependent on the combination of Mach number and dynamic pressure. Needless to say, a growing amplitude response indicates a flutter condition.

Figure 4.4 shows the stable, neutrally stable, and unstable responses of the displacement at the center chord of the tip section for dynamic pressures of $1.32 \mathrm{psi}, 1.34$ psi, and 1.36 psi respectively at a freestream Mach number, $M_{\infty}=0.795$. Computations for obtaining the dynamic responses of the wing were performed with a time step equal to $1 / 24$ of the period of the first bending mode of the structure. Though this time step is not small enough to resolve the responses of higher than the second lowest frequency modes, it was reasonable to detect flutter boundaries because the critical mode in the experiment was the first bending mode and there was no frequency coalescence. A total of 50 multigrid cycles was determined to be enough to converge the inner iteration of each time step by 4 orders of magnitude with the Courant-Friedrichs-Lewy (CFL) number of 2.

As seen in the Figure 4.4, a freestream pressure of 1.32 psi gives a decaying amplitude curve for the deflection which gradually goes to zero. This gradual decay indicates that the corresponding dynamic pressure is below the flutter point. The 


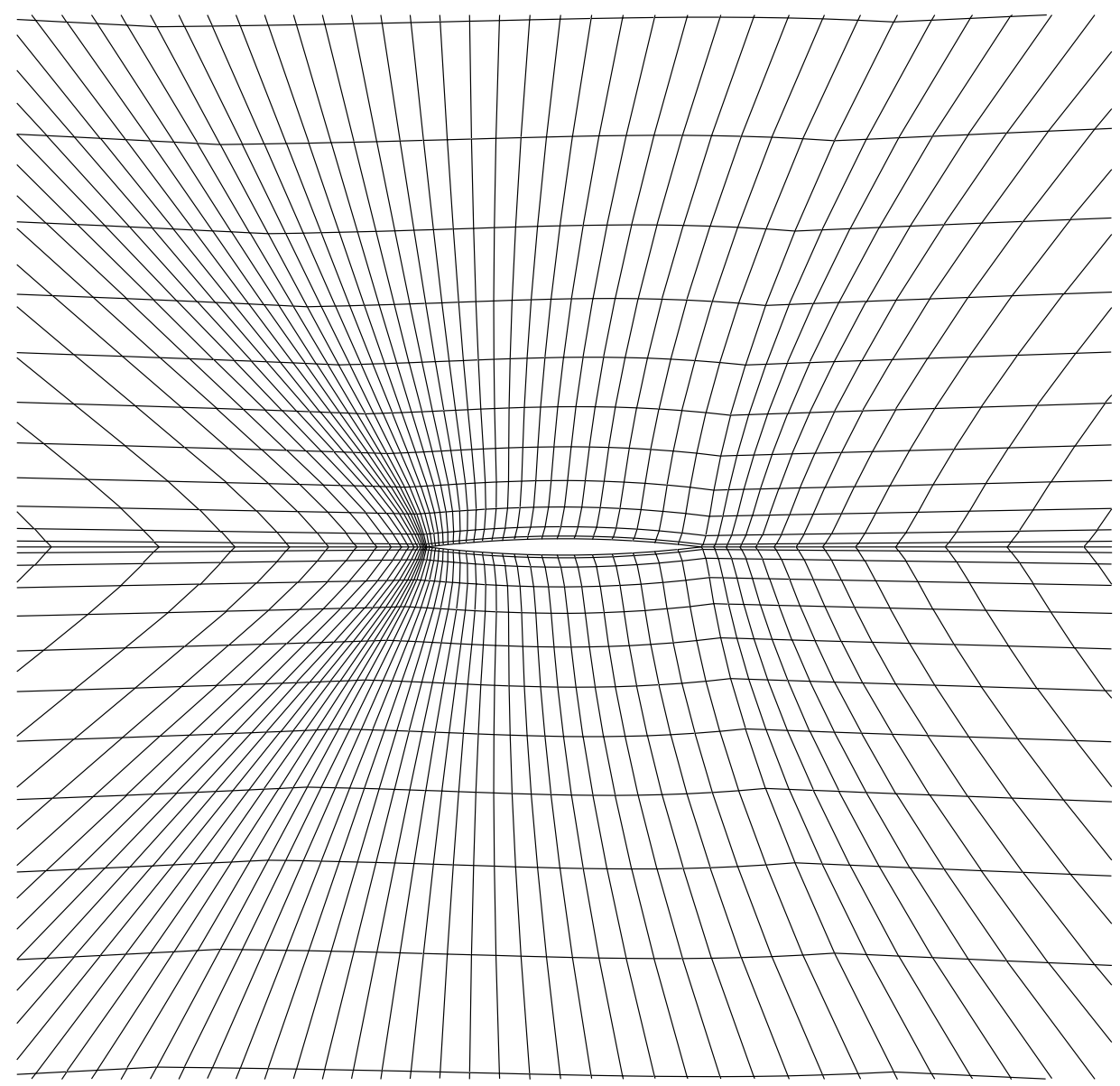

Figure 4.3: Computational Grid for the Cantilever Double Circular Arc Airfoil Wing 

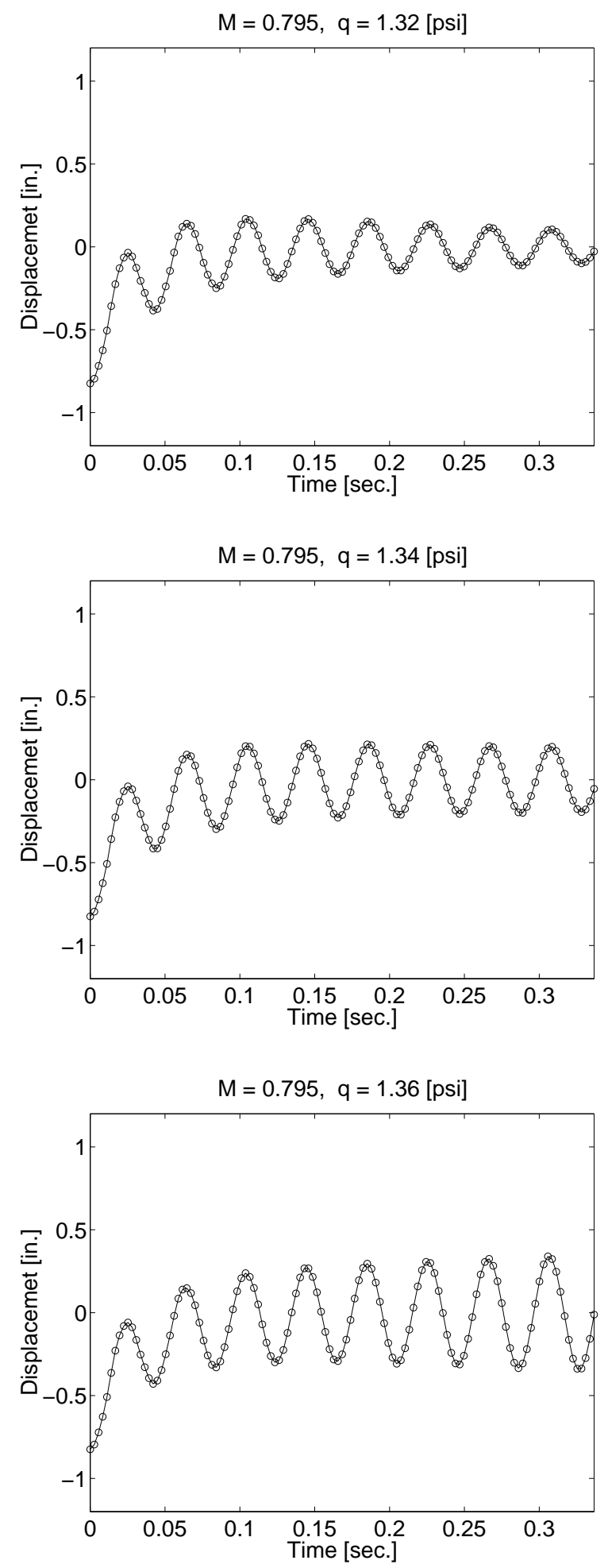

Figure 4.4: Time History of the Displacements at the Mid-Chord of the Tip 
frequency of the vibration in the figure is faster than that of the first bending mode, but it can be said that the vibration is dominated by the first bending mode and the frequency change is a usual trend when a dynamic pressure approaches to the flutter point. The second figure shows the structural response when the dynamic pressure was changed from 1.32 psi to 1.34 psi. In this figure, the decay of the amplitude of the tip deflection of the wing becomes quite a bit slower than the case of 1.32 psi and the time history in the figure looks like an almost constant amplitude motion. This can be recognized as the flutter point by the definition that flutter occurs when the damping ratio of the response of the wing becomes less than zero. This time history can be generated by an almost perfect balance between the unsteady aerodynamic loads acting on the wing and the structural response of the wing in keeping a constant amplitude of the vibration. The flutter frequency is higher than that of the first bending mode, but the mode exhibiting the flutter is apparently the lowest frequency mode, in this case the first bending mode, as is often the case. Finally, when the dynamic pressure goes beyond the flutter point, the structural response consists of a divergent vibration as seen in the case of 1.36 psi in Figure 4.4. Once flutter occurs as seen in the figure, the structure would immediately fail due to the large amplitude motion unless the flow condition around the wing changed significantly. The experimental dynamic pressure measured at the flutter boundary for this Mach number is 1.26 psi. In the same way, flutter boundaries for other Mach numbers are detected by looking at time histories of the tip deflections of the wing.

Since the only parameter changed to detect the flutter boundaries is the dynamic pressure, flutter boundaries can be shown on a figure where the corresponding dynamic pressures are plotted against Mach number. The results of the flutter boundaries determined by the computational responses are compared with the experiment [94] in Figure 4.5. Each flutter point was determined as the dynamic pressure corresponding to zero damping ratio based on a linear interpolation between one simulation 


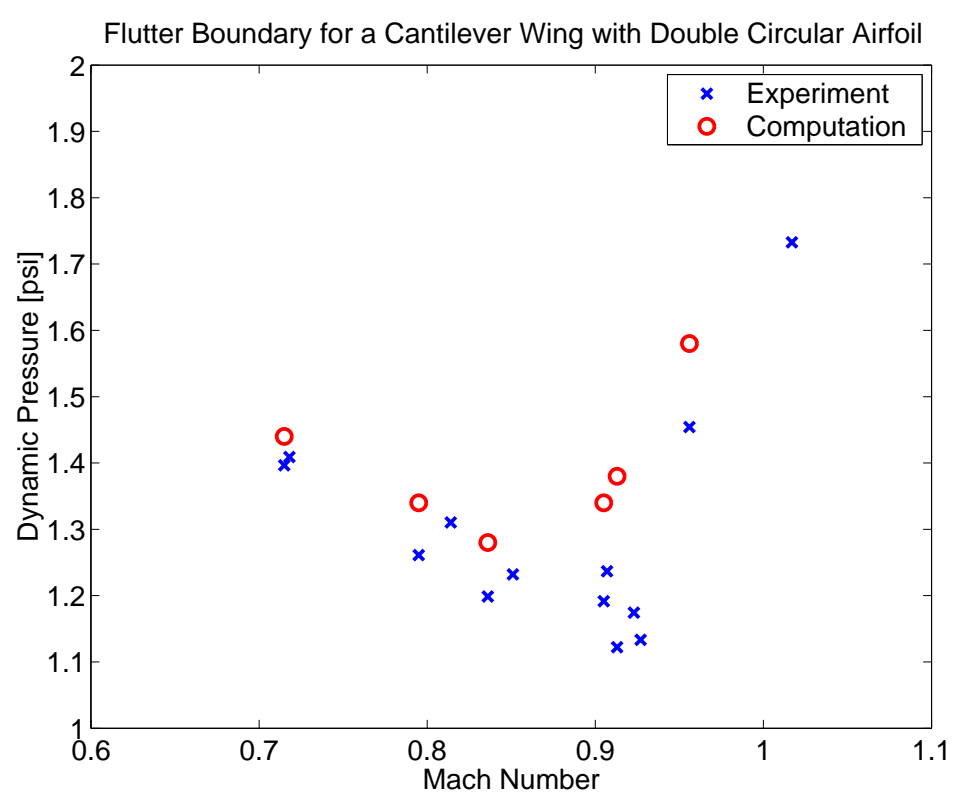

Figure 4.5: Dynamic Pressure at the Flutter Onset for Various Mach Number

above the flutter point and one below the flutter point. The variation of the flutter onset dynamic pressure with Mach number results in an almost linear decrease until a value near $M_{\infty}=0.9$ and then, an increase. As shown in the figure, there is a marked difference between the flutter boundaries of the experiment and the computations in the magnitude of the value of the flutter onset dynamic pressure near $M_{\infty}=0.9$. The difference in flutter boundaries must be primarily caused by the error in the first natural frequency of the structural model, which is nearly 5 percent, and it should also be caused by the lack of viscous effects in the model. When the amplitude of oscillation becomes large, as seen in conditions near or beyond the flutter point, the flow may separate from the wing especially near the shock for transonic cases. Thus, the inviscid flow assumption cannot work properly to execute a perfect simulation. However, It can be said that the aeroelastic solver can predict the flutter boundary fairly well even with the Euler flow solver. 


\subsection{Flutter Analysis Demonstration - NASA Ro- tor 67}

\subsubsection{NASA Rotor 67}

The purpose of this study is to demonstrate the capability of the present analysis program for predicting the flutter boundary of a realistic turbomachinery fan. As pointed out in Chapter 1, supersonic flutter can occur even on the design speed line. This type of flutter features strong shock oscillations and shock induced separations. The potential interaction between these nonlinear flow features and the structural response requires flutter explorations. Therefore, the goal of this study is to predict the flutter boundaries in the compressor performance map, to examine the influence of the inter-blade phase angle, and to observe how the flow field affects the instability of the blade vibrations.

The selection of the configuration on which aeroelastic calculations are performed is difficult due to the fact there is no turbomachinery rotor configuration whose geometry, structural properties and experimental results for flutter boundaries are available in the open literature. Because of this the predictions presented here can not demonstrate the accuracy of flutter boundary prediction. It is, however, hoped that these calculations will be compared with other numerical predictions and become a standard test case in this field.

NASA Rotor 67, the first stage rotor of the NASA Lewis (now NASA Glenn) designed two-stage fan [96], has been recognized as a popular test case for threedimensional viscous flow prediction procedures because of the available detailed experimental data obtained using a laser anemometer [41]. In the early 1990's, many researchers reported steady flow predictions for this configuration [97, 98, 99, 100, 101]. Although the structural properties for this configuration are not available, the blade is considered to be flexible enough for flutter to occur under some flow conditions. 
Rotor 67 has been used to validate aeroelastic applications by a few researchers. Chuang and Verdon [102] applied their aeroelastic analysis code, called TURBO, to this configuration. They examined the stability of the rotor blade in an inviscid flow with the work per cycle responses to a prescribed blade vibration corresponding to a reduced frequency of 0.54 based on the mid-span section. The reduced frequency, $k$, is one of the most important parameters for flutter analysis defined by the following expression.

$$
k=\frac{c \omega}{2 V_{\text {ref }}} .
$$

Here, $\omega$ is the frequency of the vibratory motion of the blade. For fans or compressors, the relative inlet velocity at a certain blade section is chosen for $V_{\text {ref }}$. The variable $c$ is the chord length of the corresponding blade section. Chuang and Verdon showed the blade to be more stable when operating at the near stall point than at the near peak efficiency point for a torsional mode. They also discussed the role of the unsteady forces around the passage shock in determining the stability of the blade motion. The location of the shock on the blade surface and the inter-blade phase angle are especially thought to have a significant effect on whether the unsteady flow extracts energy from the blade motion. L. He [40] has examined Rotor 67 vibrating with a prescribed torsional mode with a Thin-layer Navier-Stokes aeroelastic solver. The oscillation frequency had a reduced frequency of 1.5 based on the tip section. He showed a significant effect of the viscous and tip clearance flow on the unsteady torsional moments. As mentioned in Chapter 1, Vahdati, Imregun and their colleagues [52] performed a fluid/structure coupled aeroelastic computation for Rotor 67 with a Galerkin discretization of the fluid equations and ANSYS as the structural solver. The reduced frequency of their calculations was 0.324 based on the tip section. Their results show a rapid decay of the blade vibration at the near peak efficiency operation point. Thus, Rotor 67 has been gaining in popularity as a test case among 


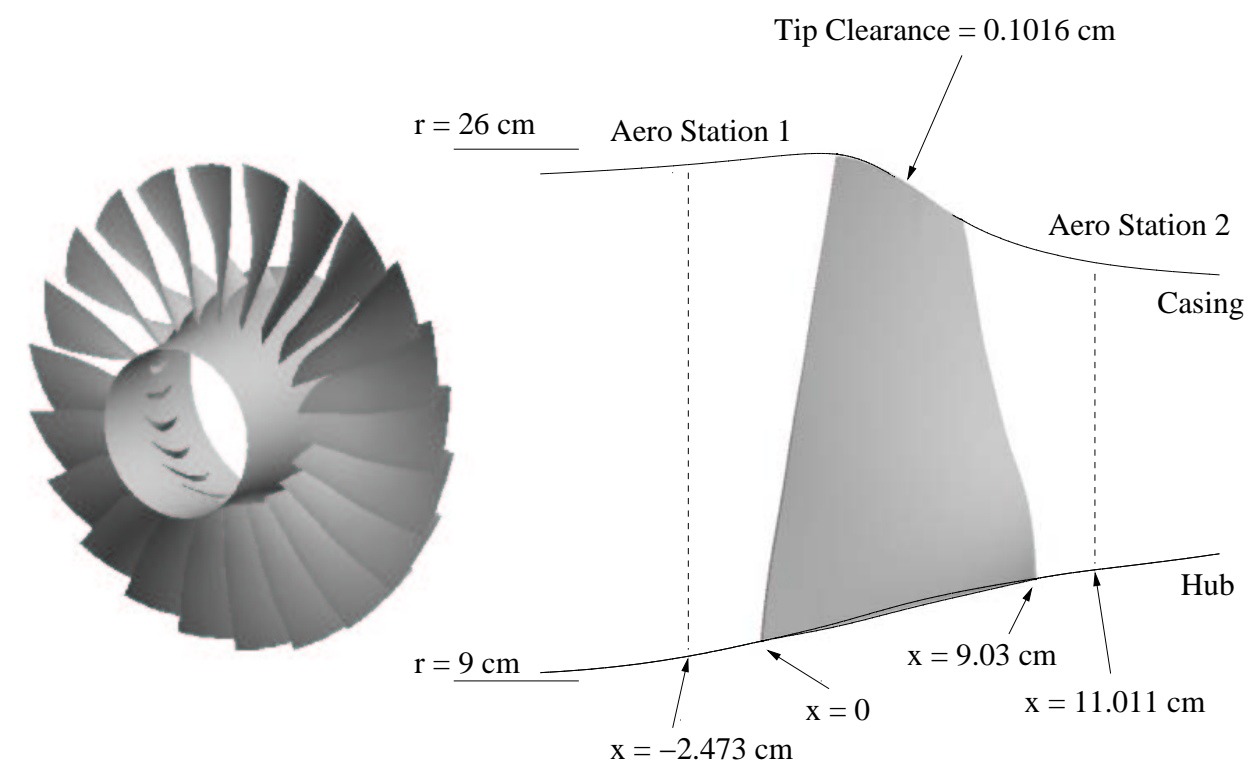

Figure 4.6: NASA Rotor 67

researchers interested in turbomachinery aeroelasticity in spite of the lack of available data regarding its flutter characteristics. For these reasons, Rotor 67 was chosen for the current investigation to demonstrate the capability of the previously described nonlinear aeroelastic prediction procedure.

NASA Rotor 67 consists of 22 blades. The blade aspect ratio is 1.56. At the design point, the rotational speed of the rotor is 16,043 RPM, with an inlet axial Mach number of approximately 0.49 , a tip inlet relative Mach number of 1.38 , a tip speed of $428.9 \mathrm{~m} / \mathrm{s}$, a total pressure ratio of 1.63 and a mass flow of $33.25 \mathrm{Kg} / \mathrm{s}$. Figure 4.6 shows an overall view of Rotor 67 with a schematic detailing some important dimensions. The rotor has a large amount of twist from hub to tip and the flow field is extremely complex featuring a three-dimensional shock structure, shock-boundary layer interaction, three-dimensional flow separation, and tip leakage flow. 


\subsubsection{Computational Grid}

Unsteady solutions are computed over single or multiple blade passages, depending on the inter-blade phase angles. These computational domains are discretized to create a multiblock structured computational grid. The grid used for the present study is shown in Figure 4.7 with the meridional view and Figure 4.8 with the blade-to-blade view. The grid for the single passage of Rotor 67 consists of 137 mesh points in the stream-wise direction, 65 points in the blade-to-blade direction, and 81 points in the span-wise direction. In the stream-wise direction, the grid extends one axial chord upstream and downstream from the blade row at the hub, and has 33, 73 and 33 axial points along the upstream periodic boundary, in the blade passage and along the downstream periodic boundary, respectively. A total of 17 points are used in the span-wise direction to describe the tip-clearance between the rotor blades and the casing.

The rotor has a $0.1016 \mathrm{~cm}$ tip clearance which makes the grid generation for a structured multiblock code quite difficult. To take this into account, the grid in the tip clearance region above the rotor tip is constructed by simply extending the blade span to the casing, creating another block between the tip and the casing. The tip clearance block is then discretized as shown in the Figure 4.9, making singular points at the leading and trailing edges. The finite volume procedure can integrate the flux going through the prismatic cells created at the leading and trailing edges simply setting the projected face area at the singular points to zero. Boundary condition treatment for these singular points is not required since the flux passing through these points is zero. 


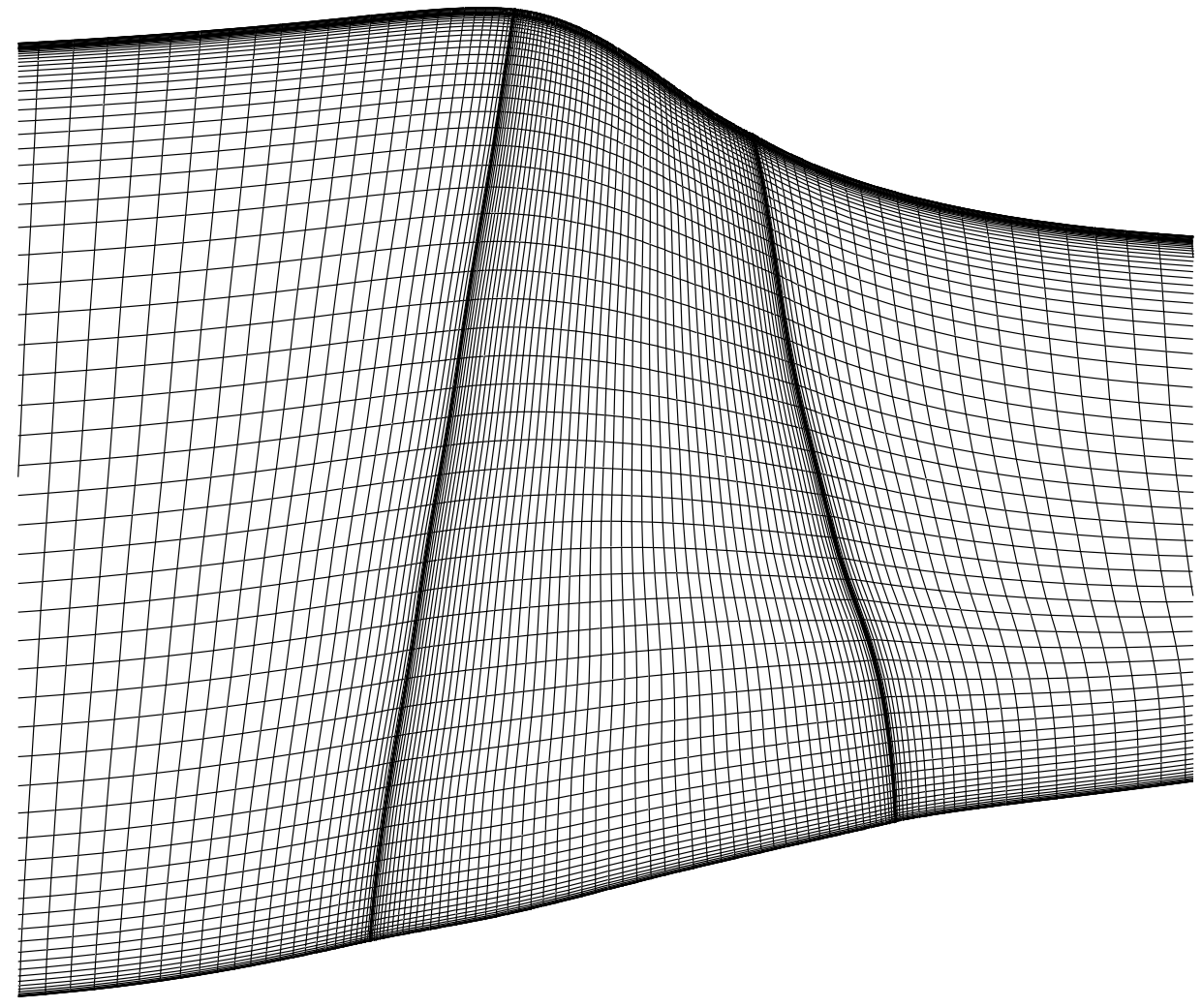

Figure 4.7: Meridional View of Computational Grid for NASA Rotor 67 


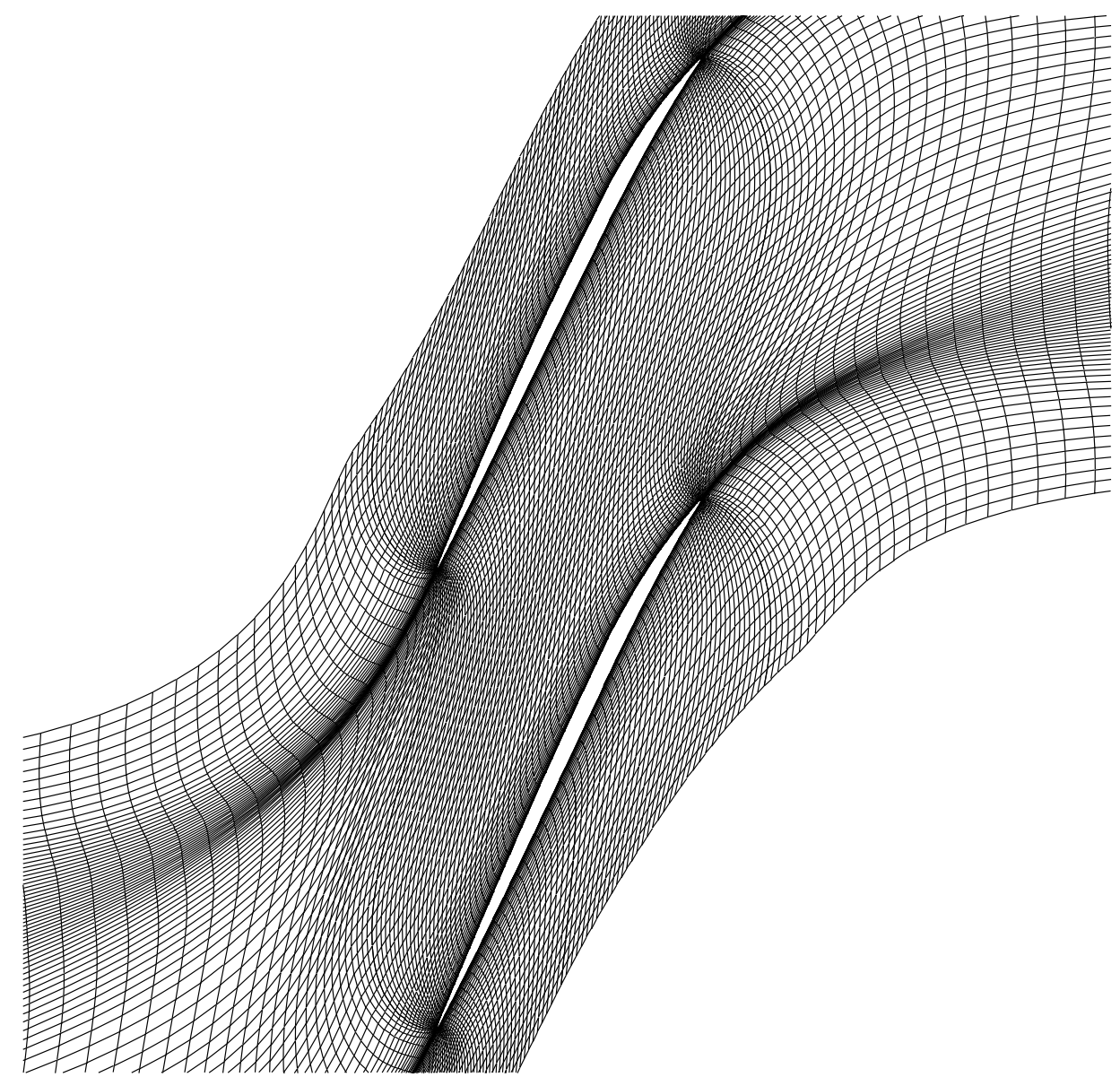

Figure 4.8: Blade-to-Blade View of Computational Grid for NASA Rotor 67 


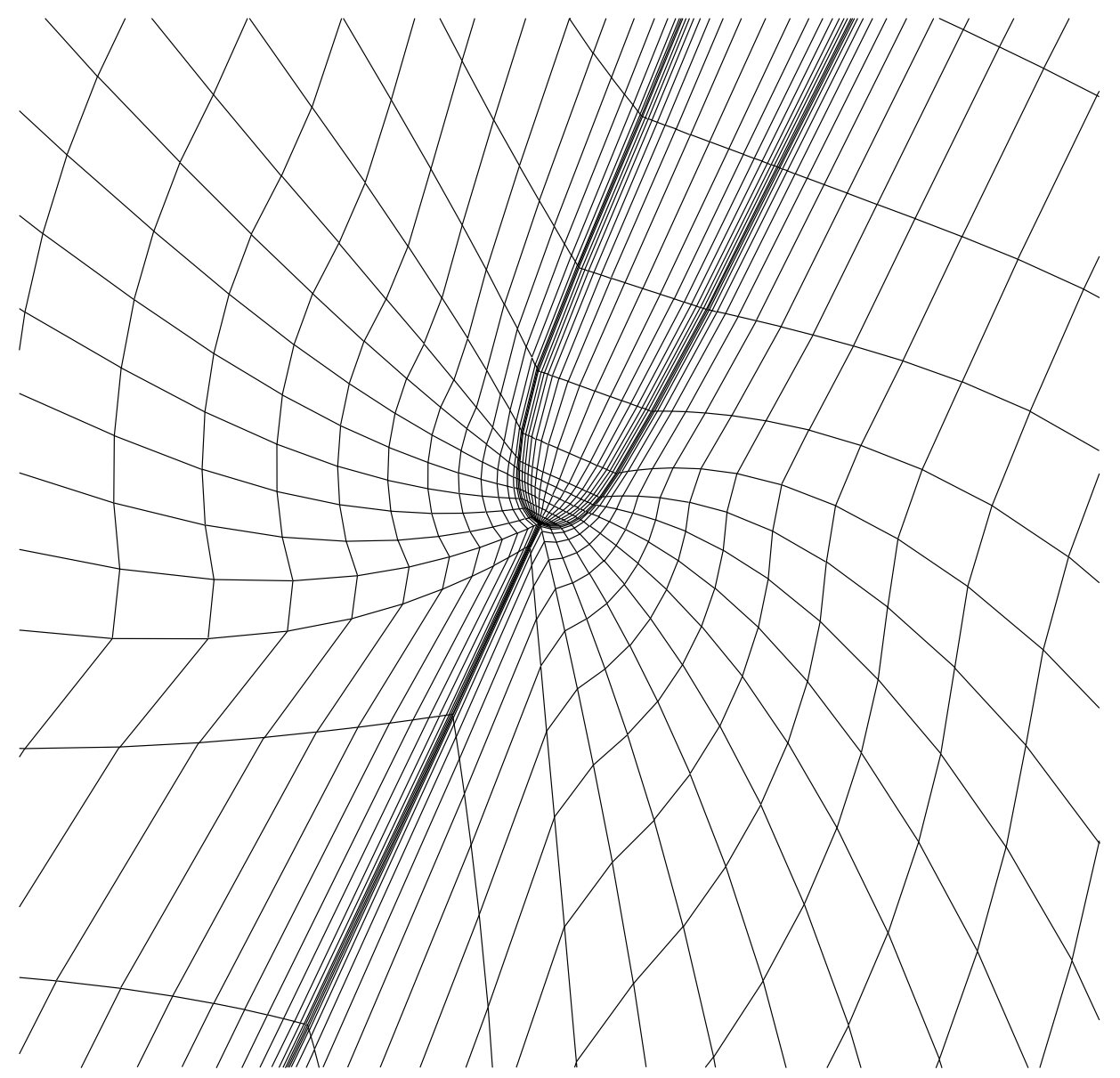

Figure 4.9: Grid Near the Tip Clearance 


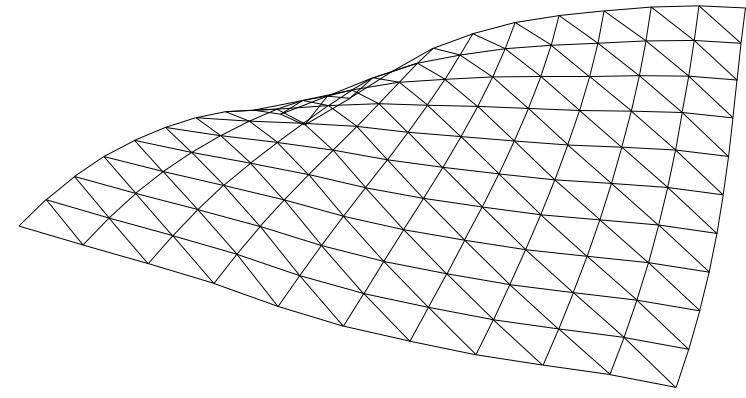

Figure 4.10: Finite Element Model of the NASA Rotor 67

\subsubsection{Blade Structural Model}

A NASTRAN finite element model was used to calculate direct transient structural responses as well as to obtain the mode shapes and frequencies of the blade. The model for the blade shown in Figure 4.10 consists of $11 \times 11$ nodes forming 200 triangular plate elements with varying thickness. The thickness is distributed to match the actual thickness of the blade. For structural boundary conditions, both translational and rotational displacements are not allowed at the root of the blade.

Materials for blade construction are conventionally aluminum alloys, steel or stainless steel. However, in recent applications Titanium and later Beryllium have become significant. In modeling NASA Rotor 67, an imaginary material is used, whose properties are shown in Table 4.1 with that of a titanium alloy. The Young's modulus and the density are chosen such that the first natural frequency becomes around 400 Hz. The reason why $400 \mathrm{~Hz}$ is chosen comes from the usual elastic design procedure of rotor blades. The basic principle that needs to be satisfied in the design procedure 
Table 4.1: Material Properties

\begin{tabular}{cccc}
\hline & Young's modules & Poisson's ratio & Density \\
\hline \hline Titanium Alloy & $1.172 \mathrm{e}+11[\mathrm{~Pa}]$ & 0.3 & $4539.5\left[\mathrm{~kg} / \mathrm{m}^{3}\right]$ \\
\hline Current Material & $1.422 \mathrm{e}+11[\mathrm{~Pa}]$ & 0.3 & $4539.5\left[\mathrm{~kg} / \mathrm{m}^{3}\right]$ \\
\hline
\end{tabular}

is that resonant response between the blade mode and the rotation speed must be avoided. The design rotation speed for Rotor 67 is $267.4 \mathrm{~Hz}$ and its double is 534.8 Hz. A typical first natural frequency of the blade is placed between these two numbers to give adequate margin. However, as pointed out by Kielb [103], it is very difficult to accomplish this for a solid titanium blade. In fact, if the blade of Rotor 67 was modeled with a solid Titanium alloy with material properties described in the table, the first frequency at the design speed would become $520 \mathrm{~Hz}$ which is very close to the double of the rotation speed. Therefore, $400 \mathrm{~Hz}$ is picked as the first natural frequency in order to keep a satisfactory margin with respect to the fundamental frequency of revolution and its integer multiples.

The calculated mode shapes and modal frequencies for the Rotor 67 structural model with the centrifugal stiffening included are shown in Figure 4.11 with their frequencies. The blades are seen from the outer casing towards the axis of the rotor. To obtain the mode shapes and frequencies, an eigenanalysis solution sequence 103 in MSC/NASTRAN was used. In the figure, the blades drawn with thinner mesh lines represent the original rigid shape of the blade, and the other solid pictures show the corresponding mode shapes. The behavior of every mode is similar in terms of its torsional motion near the root and bending motion near the tip. In addition, more torsional components near the tip can be observed in the higher frequency modes especially in the third and fourth mode.

For the design rotation speed of Rotor 67, the reduced frequency based on the 


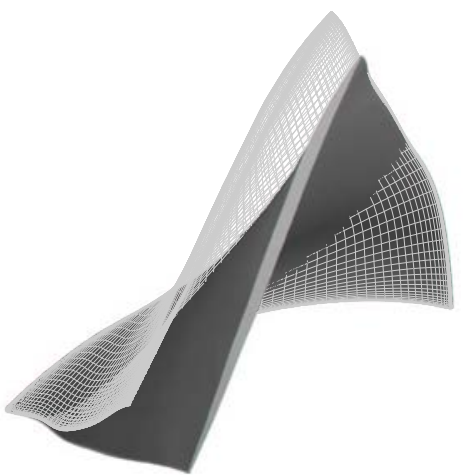

(a) First Mode - $401.9 \mathrm{~Hz}$

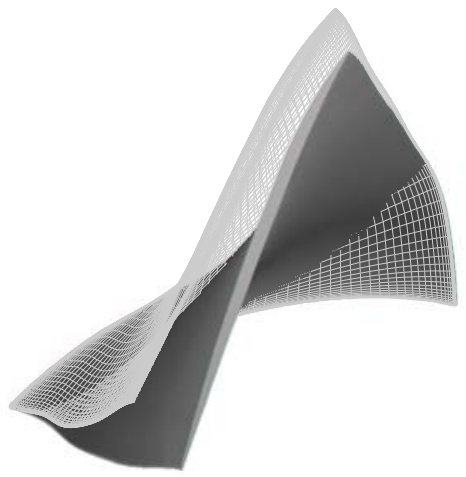

(c) Third Mode - $2093.7 \mathrm{~Hz}$

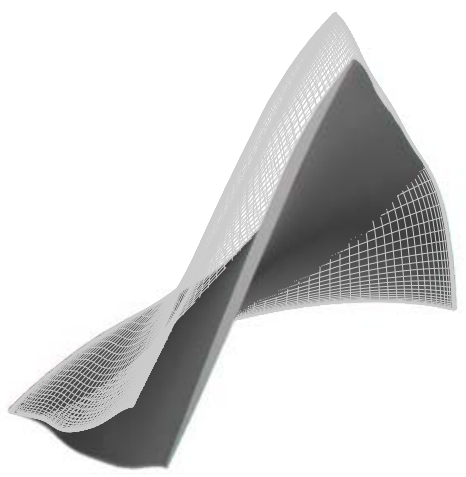

(b) Second Mode - 1096.0 Hz

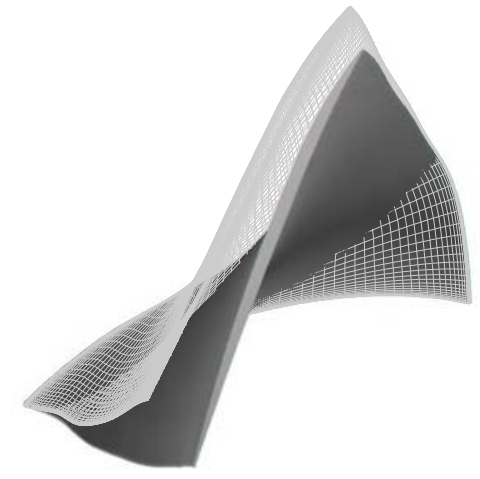

(d) Forth Mode - 2456.6 Hz

Figure 4.11: Mode Shape of the Rotor 67 
tip section becomes 0.27 according to the first mode frequency calculated with the structural model by MSC/NASTRAN. Finally, for multi-passage simulations, the cascade is assumed to be well tuned, which means that all the blades have identical structural properties.

\subsubsection{Steady Flow}

Prior to performing the aeroelastic unsteady calculations, validation of the steady flow solution needs to be performed such that the proper initial conditions for unsteady calculations can be provided. In order to obtain an accurate representation of the viscous flows around the rotor, the three-dimensional RANS equations are solved in a relative non-Newtonian reference frame rotating with the blade. The $k-\omega$ model is used to predict the turbulent eddy viscosity in the flow. Validation is conducted by comparing the solutions with measurements taken with a laser anemometer [41] and other RANS numerical simulations performed by numerous researchers. For example, Chima [97] and Ha, et al. [98] gave detailed descriptions of the three-dimensional flow structure around the blade. Jennions, et al. [99] and Adamczyk et al. [100] examined the influence of the tip clearance by changing its size in the radial direction. Arnone [101] described the complex nature of the transonic flow through Rotor 67 in detail.

Steady flow computations are performed with the measured total pressure, total temperature and absolute flow angle profiles specified at the inlet and the measured static pressure profile specified at the exit. Computations take approximately 2000 iterations with a CFL of 3 and three levels of a multigrid W-cycle to reach converged solutions where the average density residual decreases by 3.5 orders of magnitude from the initial conditions. The same CFL number is used for integrating the turbulence model equations. The predicted solution at the near peak efficiency point is used as initial conditions for all of the simulations which were run an additional 1000 to 
2000 iterations with different inlet and exit profiles depending on the operating point. More iterations tend to be necessary for convergence for solutions closer to the stall condition.

To show the ability of the flow solver to capture the correct performance, the numerically calculated performance map of the rotor is shown in Figure 4.12 along with the experimentally measured values. A total of 12 different operating conditions were calculated to asses the overall accuracy in the numerical prediction of performance. The adiabatic efficiency and total pressure ratio are calculated using the mass averaged values of the total pressure and total temperature at the inlet and exit aero survey stations shown in Figure 4.6. The adiabatic efficiency is defined as,

$$
\eta=\frac{\left(P_{t 2} / P_{t 1}\right)^{(\gamma-1) / \gamma}-1}{\left(T_{t 2} / T_{t 1}\right)-1},
$$

where $P_{t 2}$ and $P_{t 1}$ are the absolute total pressures at the inlet and exit respectively, and $T_{t 2}$ and $T_{t 1}$ are the corresponding absolute total temperatures. The mass flow in Figure 4.12 is nondimensionalized with respect to the choking mass flow. The mass flow rate at the choke condition is found to be about 1.8 percent lower than the experimentally observed value. An underprediction of the choked mass flow rate is also reported in the other computational results $[97,99,101]$. As shown in Figure 4.12 , the overall predicted performance of the compressor is in good agreement with the experiment except for the following two areas. One area is that the computation underpredicted the adiabatic efficiency for the peak efficiency point and also predicted a lower mass flow rate. This trend was also predicted by Arnone [101]. The other area is the obvious underpredicted absolute total pressure ratio. This underprediction is likely due to an overprediction of shock and viscous flow losses and flow deviation. However, the steady-state solution including the structural deformation mentioned in the next section results in a higher mass flow and higher pressure ratio. Although the structural model is not based on the real structure of Rotor 67, this suggests that 

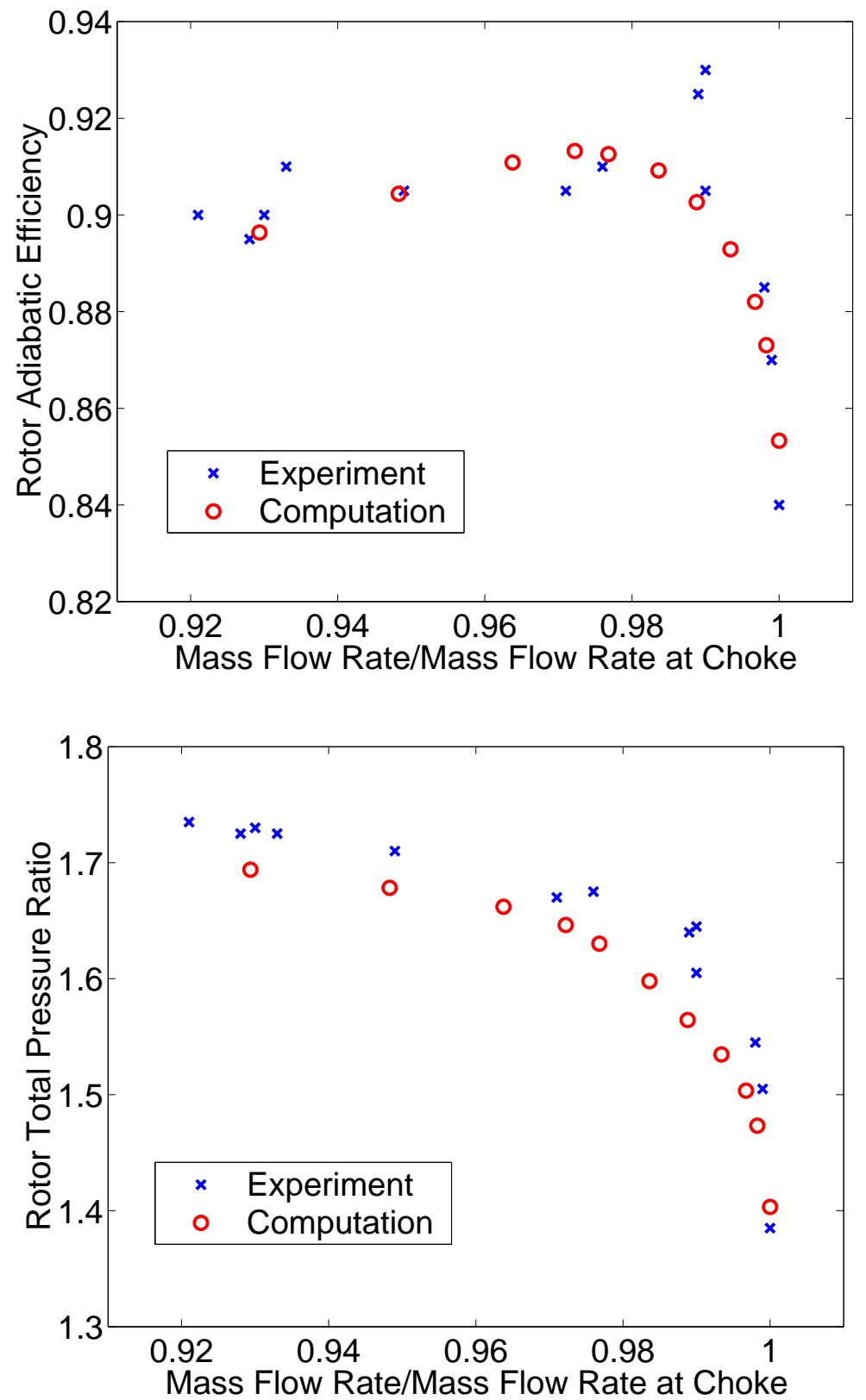

Figure 4.12: Comparison of Rotor Performance at Design Speed 
the underestimated pressure ratio and mass flow may be caused by the structural deformation not included in the present calculations.

In particular, the experimental data reported in Strazisar's document [41] concentrate on the near peak efficiency and near stall conditions. Detailed comparisons between calculations and experiment are made at these two operating points. In Figure 4.13, the relative Mach number contours at three different span-wise locations, i.e. 10, 30, and 70 percent span measured from the casing, are shown for the near peak efficiency condition. Note that the contours are plotted on constant percent radius surfaces, not on constant-radius surfaces. The relative Mach number at 10 percent of span is about 1.35. A bow shock at the leading edge and a normal inpassage shock near the trailing edge on the suction surface can be observed. At 30 percent of the span, a lower Mach number, i.e., approximately 1.2, moves the inpassage shock slightly forward but the shock structure is qualitatively similar. At 70 percent of the span, the inlet relative Mach number is subsonic, i.e., approximately 0.90, and a supersonic bubble appears near the leading edge on the suction surface. The laser anemometer data is known to be uncertain in the center of the wake at 70 percent span, where computed data indicates a large region of separated flow. It should be noted that the other computations also indicate flow separation on the suction side near the hub. Figure 4.14 shows the radial survey of computed and measured mass-averaged thermodynamic parameter profiles downstream of the rotor for peak efficiency operation. All the computed exit quantities agree very well with the measurements.

Figures 4.15 and 4.16 show detailed results at an operating point near stall. Figure 4.15 shows a comparison of relative Mach number contours. The flow structure at the near stall operating condition is similar to that for the near peak efficiency. While the near peak condition generates two shocks, i.e. a bow shock at the leading edge and an in-passage shock near the tip, a single detached bow shock is observed at the near stall 


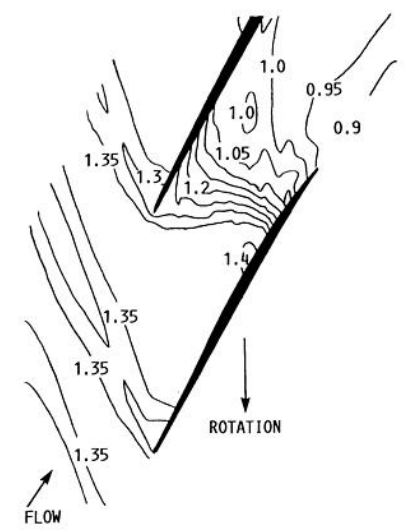

10\%Span

From Casing

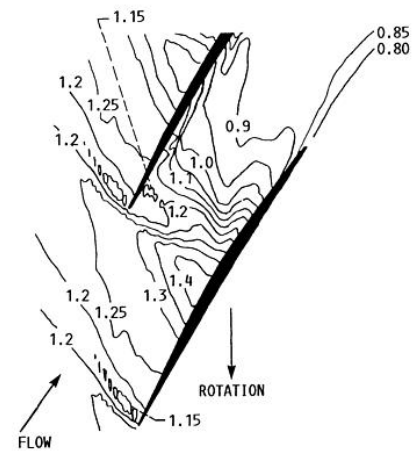

30\% Span

From Casing

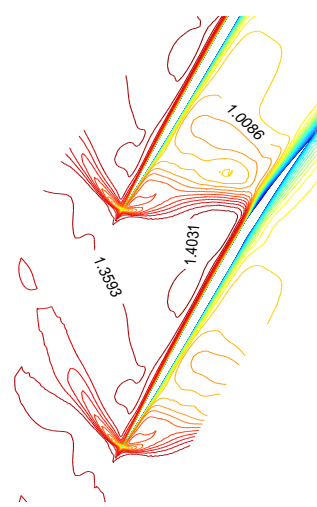

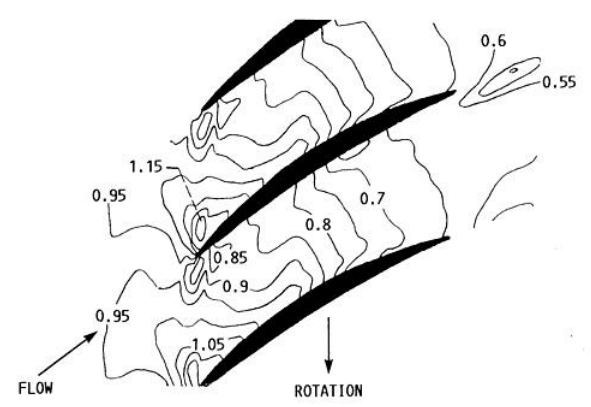

Experiment
70\%Span

From

Casing
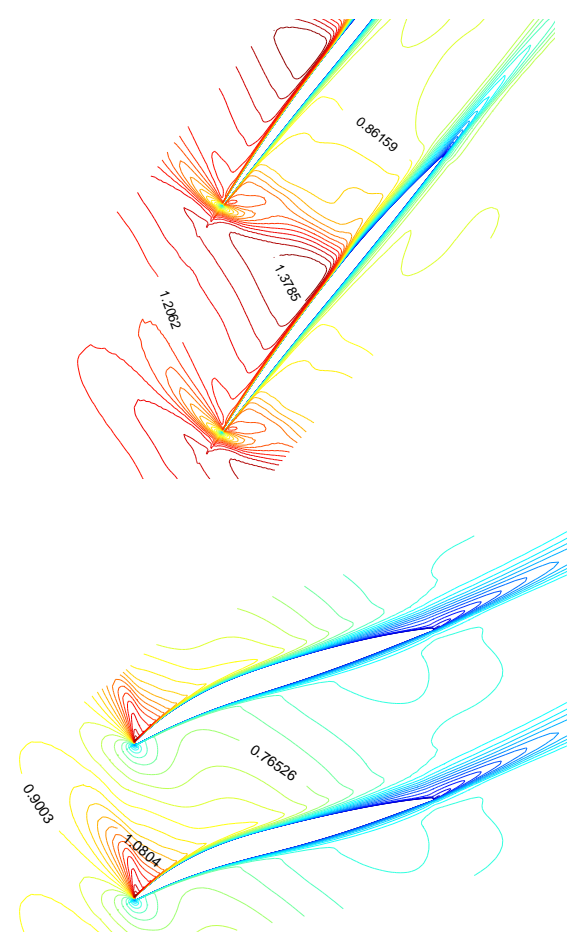

Computation

Figure 4.13: Experimental and Numerical Relative Mach Number Contour for Near Peak Efficiency 

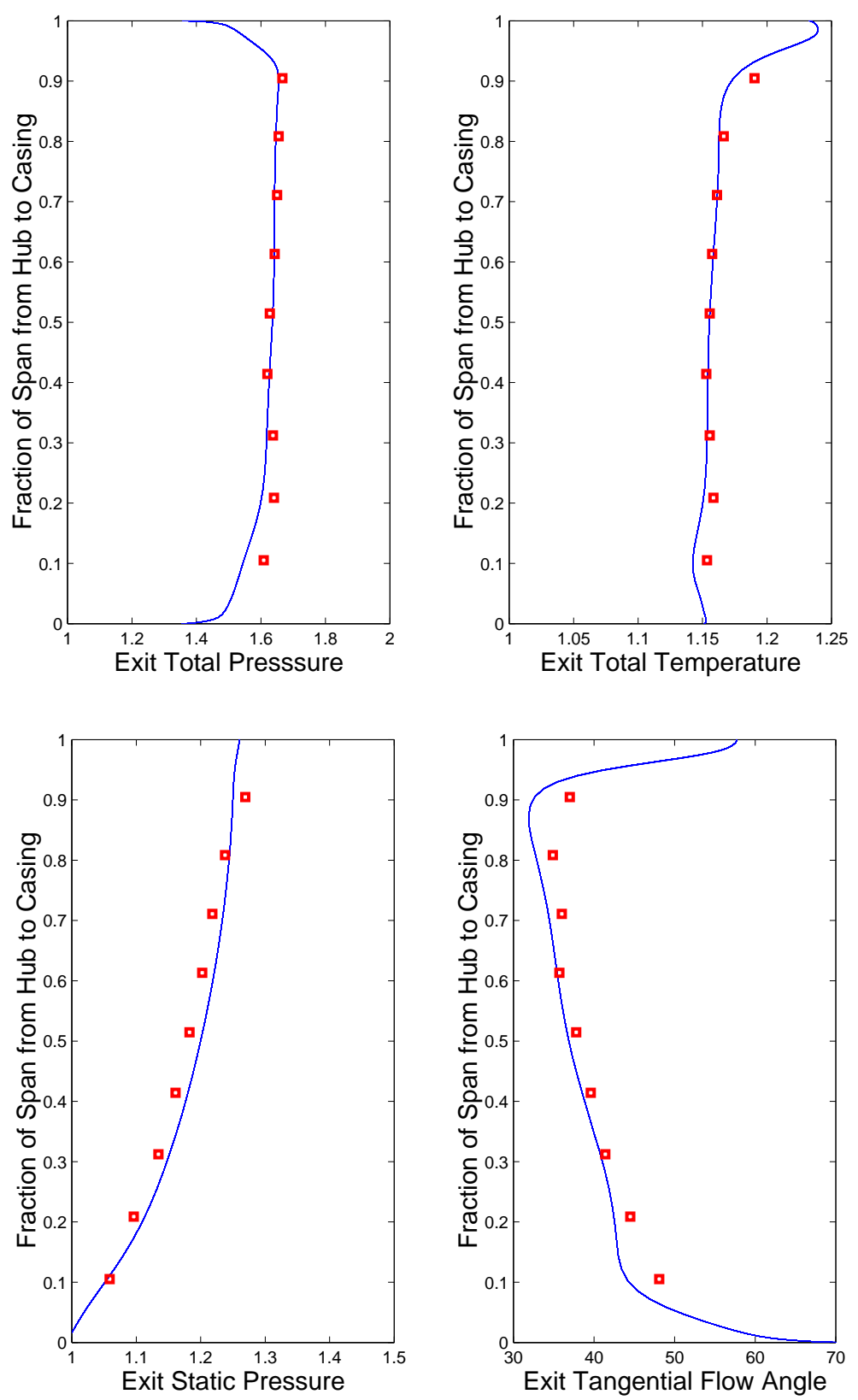

Figure 4.14: Flow Variable Distributions in the Span-Wise Direction at the Downstream of the Blade for Near Peak Efficiency 
condition. Thus, the pressure side is shock free. The computed shock is somewhat more oblique than the measured shock especially at the 10 percent span location, but the qualitative agreement is satisfactory. At 70 percent span, the supersonic bubble is very similar, but again a large area of flow separation can be observed in the computational results which does not appear in the experiment. Figure 4.16 compares radial surveys downstream of the rotor. All the computed parameters shown in the figure agree well with the experimental results.

In general, the qualitative agreement between the experiments and computations for both operating conditions is reasonably satisfactory, especially in the radial distribution of the thermodynamic quantities. As stated, the purpose of this study on the steady-state solutions is to confirm the suitability of the flow solver and the computational grid for an aeroelastic simulation. The results presented above indicate that both are suitable for predicting the proper pressure loadings and shock positions. Discrepancies between the computations and experiments are judged to be sufficiently minor so as not to affect the conclusions obtained from the aeroelastic simulations that follow.

\subsubsection{Structure-Coupled Unsteady Flow}

In this section, time dependent aeroelastic results are considered. The behavior of the blade oscillation can be determined from forced response computations. In reality, the blade-passing forced response is due to the excitation forces generated by the rotation of the bladed system past a pressure field whose strength varies periodically with the angular position of the rotor. Such flow variations are mainly caused by the stator blades which act as upstream or downstream obstructions, and the fact that the rotor blade experiences their wakes or potential field as time varying forces. In the current computations, these periodic excitations can be simulated by impulsive initial 

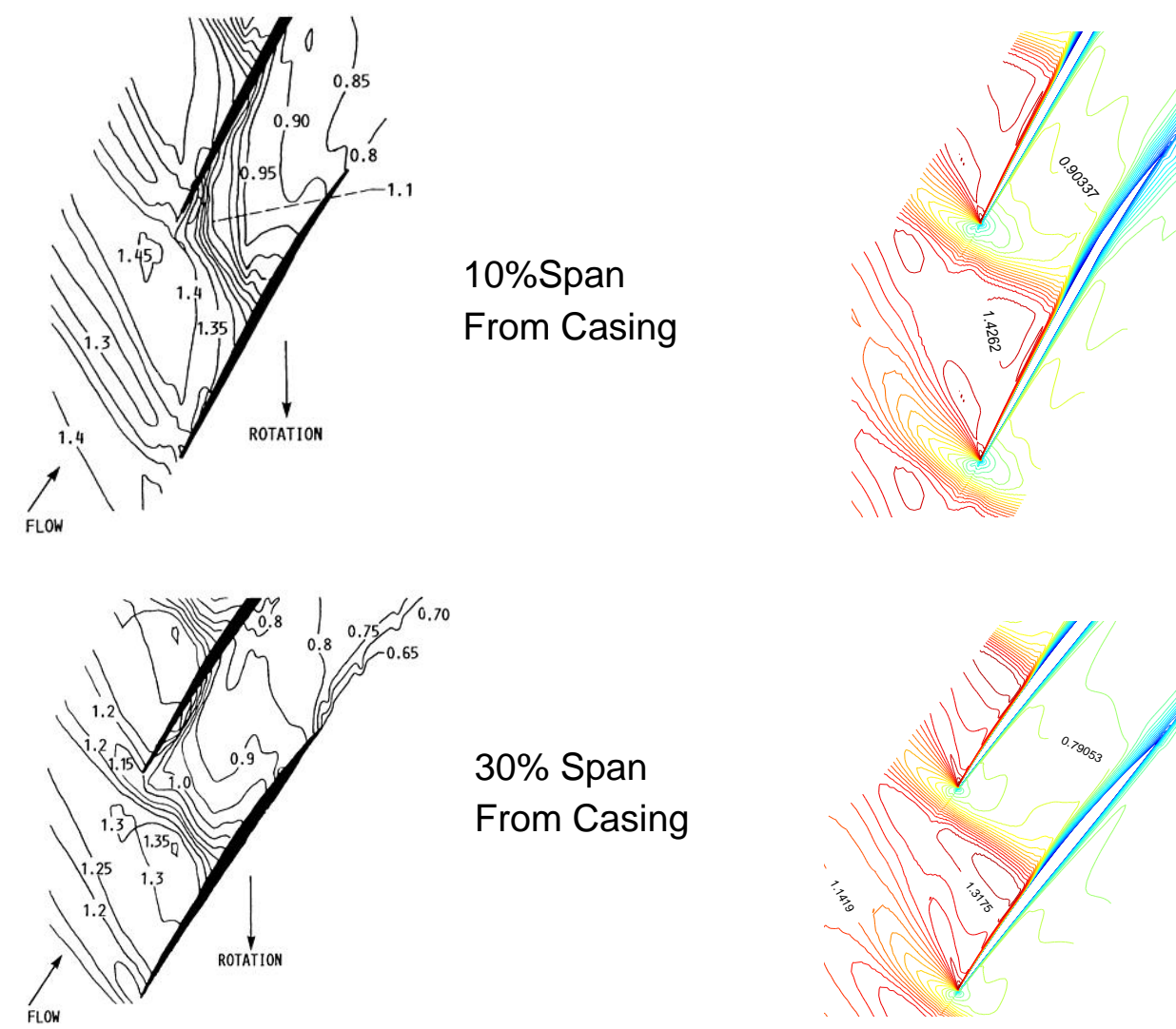

$30 \%$ Span

From Casing
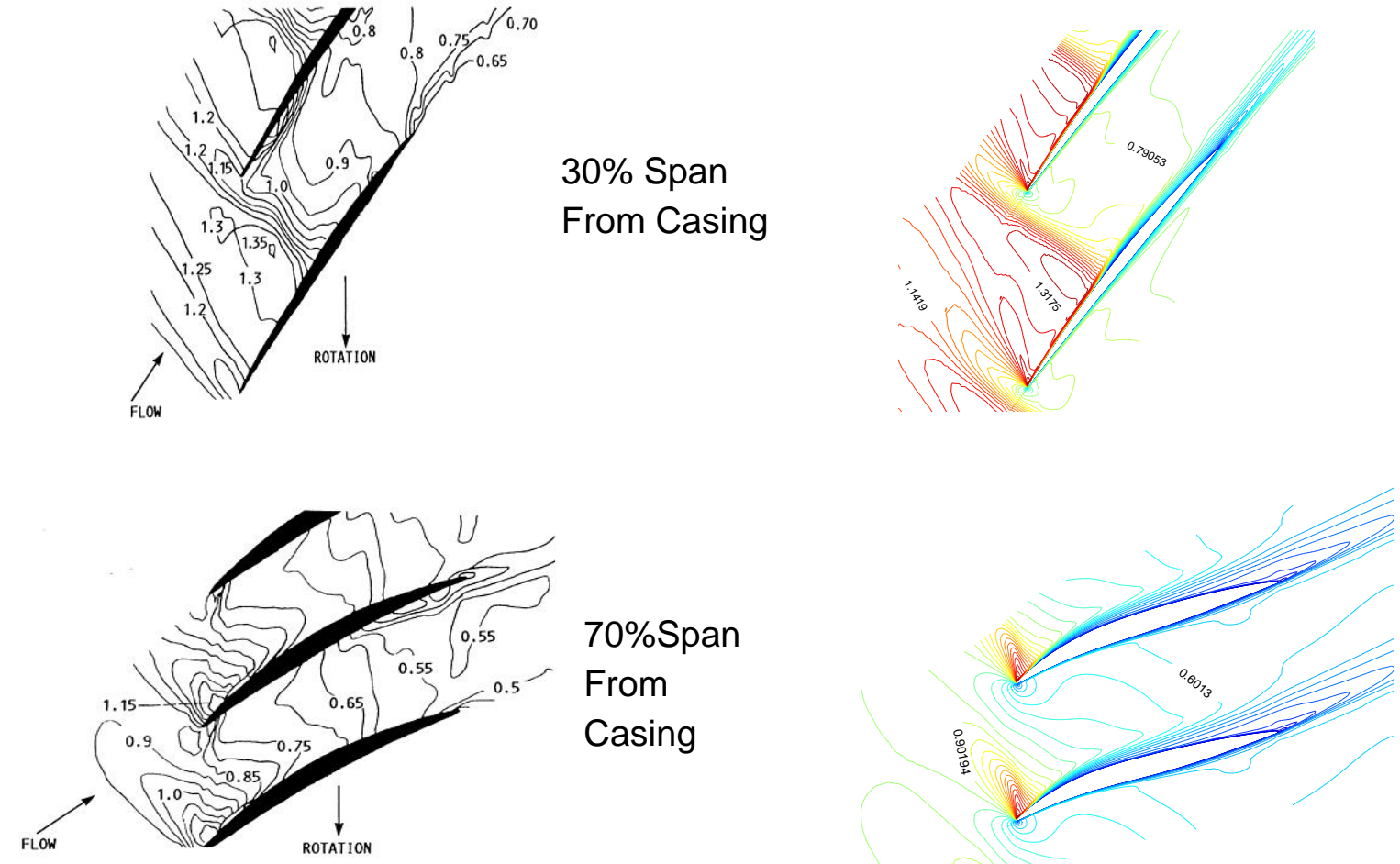

Experiment

Computation

Figure 4.15: Experimental and Numerical Relative Mach Number Contour for Near Stall 

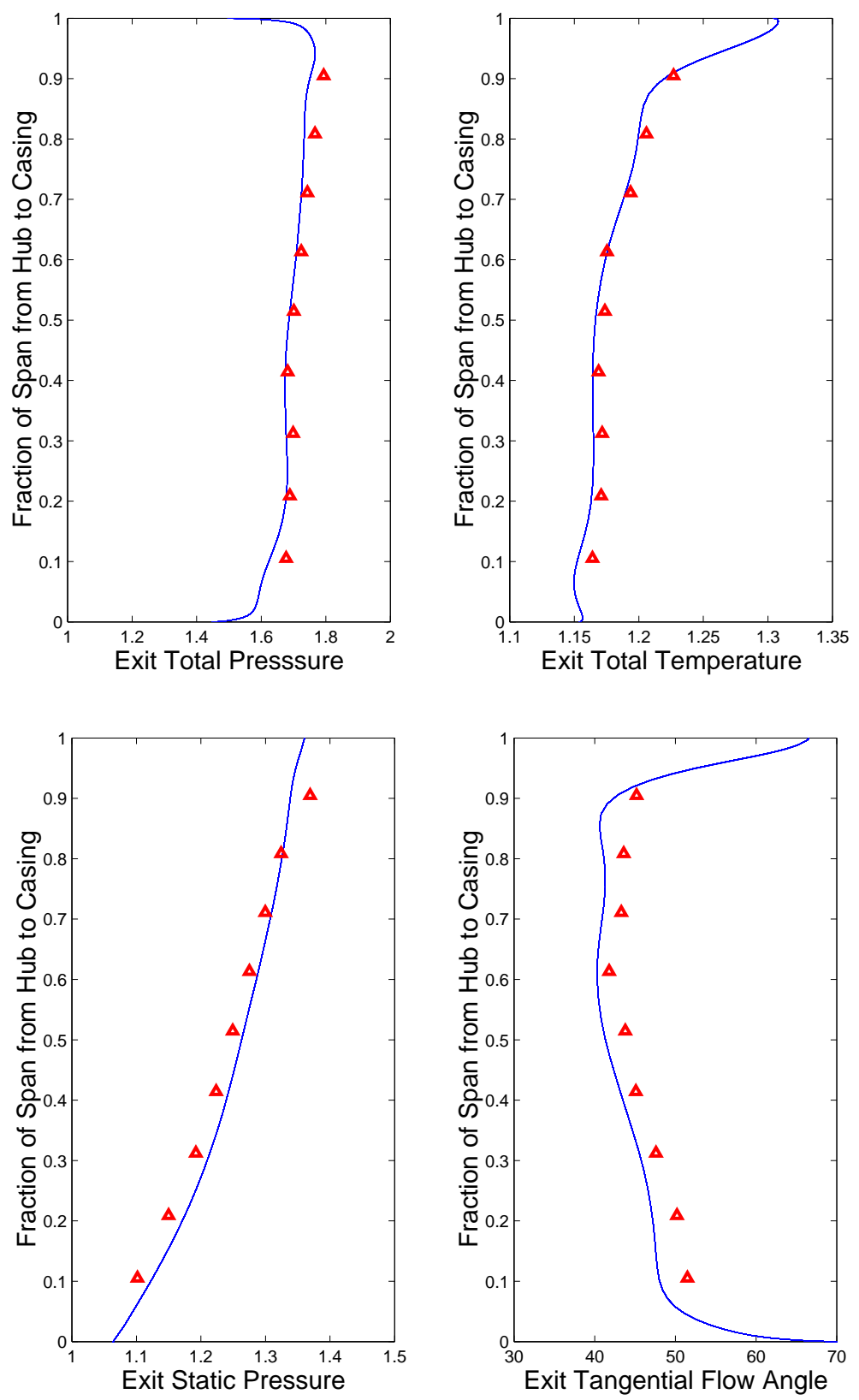

Figure 4.16: Flow Variable Distributions in the Span-Wise Direction at the Downstream of the Blade for Near Stall 
displacements given to the blade. As previously shown, the steady-state solutions can be obtained for the rigid unloaded blade geometry. Prior to the aeroelastic response calculations, the blade is allowed to move and dynamically relax to a steady-state loaded condition. The influence of centrifugal forces and fluid pressure causes the blade's steady-state loaded geometry to be different from its original unloaded geometry. Then, the blade is deformed an additional amount predominantly corresponding to the first mode. The amplitude of the blade initial deflection is about 30 percent of the deflection under steady load. Once the new steady solution for the blade with the initial deformations obtained, the blade is released and allowed to move according to the aeroelastic interaction.

The periodicity of the exciting forces is primarily determined by the ratio between the number of upstream or downstream stator blades and that of rotor blades. This periodicity results in blade vibrations propagating in the direction of rotation with a constant inter-blade phase angle $\sigma$ between adjacent blades. In the solution procedure, a constant inter-blade phase angle is determined by the initial displacements and velocities for multi-passage computations. In addition, the inter-blade phase angle is forced by the periodicity boundary condition. For example, consider the case of $\sigma=180$ degrees. The computation needs to be performed with two passages where one blade is given an initial displacement and zero initial velocity and the other is given the initial displacement in the opposite direction with the same amplitude and zero initial velocity as well. The aerodynamic force arising from the blade vibration introduces an aerodynamic damping that can be either positive or negative, depending on the operating points and the phase angle of vibration. To investigate the effect of inter-blade phase angle, calculations for four different inter-blade phase angles shown in Table 4.2 were performed at the near peak efficiency point. In addition, two interblade phase angles at a near stall operation point corresponding to the pressure ratio of 1.69 and at a near choke point with a pressure ratio of 1.5 were selected to examine 
Table 4.2: Case Matrix for Rotor 67 Aeroelastic Calculations

\begin{tabular}{ccc}
\hline Operating Point & Pressure Ratio & Inter-Blade Phase Angle $\sigma$ \\
\hline \hline Near Peak Efficiency & 1.63 & $0 \mathrm{deg}$ \\
& & $90 \mathrm{deg}$ \\
& $180 \mathrm{deg}$ \\
& $270 \mathrm{deg}$ \\
\hline Near Stall & 1.69 & $0 \mathrm{deg}$ \\
& & $180 \mathrm{deg}$ \\
\hline Near Choke & 1.50 & $0 \mathrm{deg}$ \\
& & $180 \mathrm{deg}$ \\
\hline
\end{tabular}

the effect on the flow field structure, especially the shock strength and its location.

Five cycles of oscillation, which result in about 0.02 seconds of real time, were found to be sufficient to distinguish whether the oscillation decays or grows. A total of 36 time steps per vibration period of the first oscillation mode was chosen in order to ensure the accuracy of the coupled system with the dual time stepping scheme and the Newmark scheme. Between 40 to $60 \mathrm{~W}$-cycle multigrid cycles per physical time step were necessary to provide a reasonable time-accurate pressure distribution to the structural model. These numbers were carefully determined from numerical experiments to avoid large deviations in the structural response due to the pressure fields being still in transition [95].

Flutter stability is assessed from the time history of the displacements. Figure 4.17 shows the variation of the circumferential direction displacements of the blade with time for $\sigma=0$ and 180 degrees at the near peak efficiency point. The displacements are defined as the deflections from the original rigid shape of the structure. The displacements shown in the figure are measured at the mid-chord of the tip section. Since there is no chance that a node of one of the dominant modes shown in Figure 4.11 lies at the mid-chord of the tip section,. These displacement measurements would never miss the displacements due to the dominant modes. For $\sigma=180$ degrees, only 


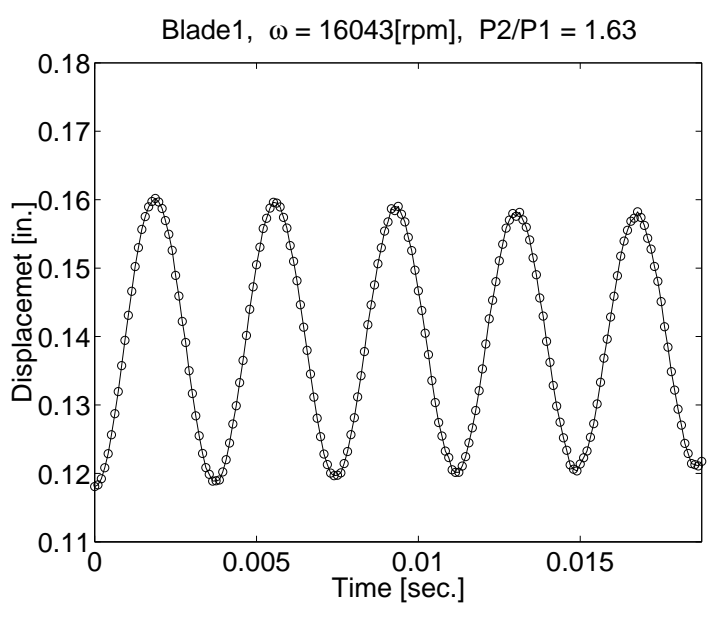

(a) $\sigma=0$ deg.

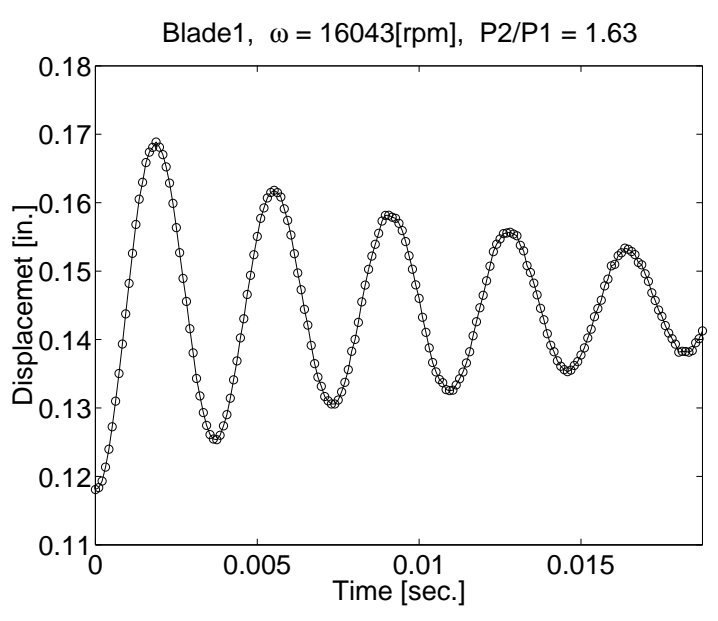

(b) $\sigma=180$ deg.

Figure 4.17: Deflection at the Mid-Chord of the Tip Section for Near Peak Efficiency, $\sigma=0,180 \mathrm{deg}$

the motion of one blade is shown. The motion of the other blade is similar except that the displacements are in the opposite direction. The response for $\sigma=0$ degrees shows almost a typical forced response with constant amplitude of oscillation. On the other hand, the response for $\sigma=180$ degree shows decreasing amplitude with each oscillation cycle, indicating a decay in the oscillation and a flutter free condition. Thus the aerodynamic damping for $\sigma=180$ degree is much greater than that for $\sigma$ $=0$ degree.

To investigate the stability for other inter-blade phase angles, calculations were started from the initial displacements and velocities corresponding to $\sigma=90$ and 270 using four passages. Figure 4.18 illustrates the responses for these inter-blade phase angles. When the inter-blade phase angle is positive, the wave of blade vibrations of a fan rotor travels in the direction of rotation as shown in Figure 1.2. In practice, a greater than 180 degree inter-blade angle produces a wave traveling in the opposite direction. All the results shown in the figure indicate decaying curves of the aeroelastic 


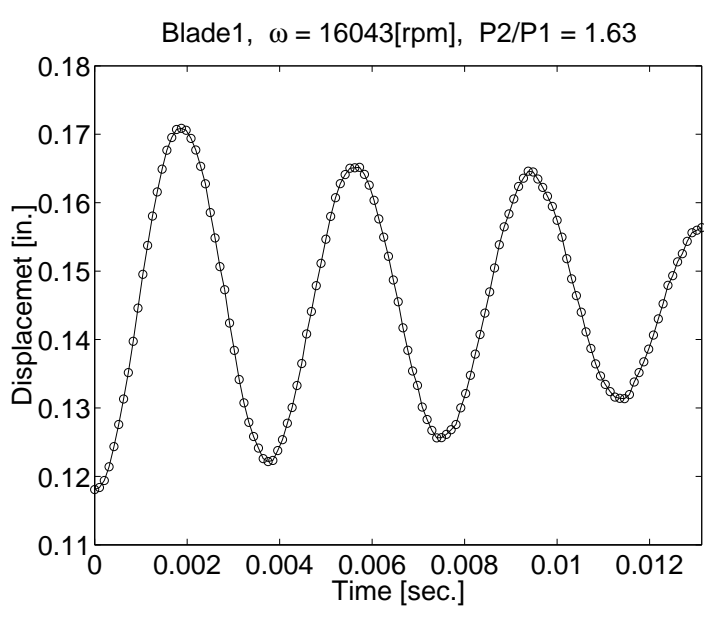

(a) $\sigma=90$ deg.

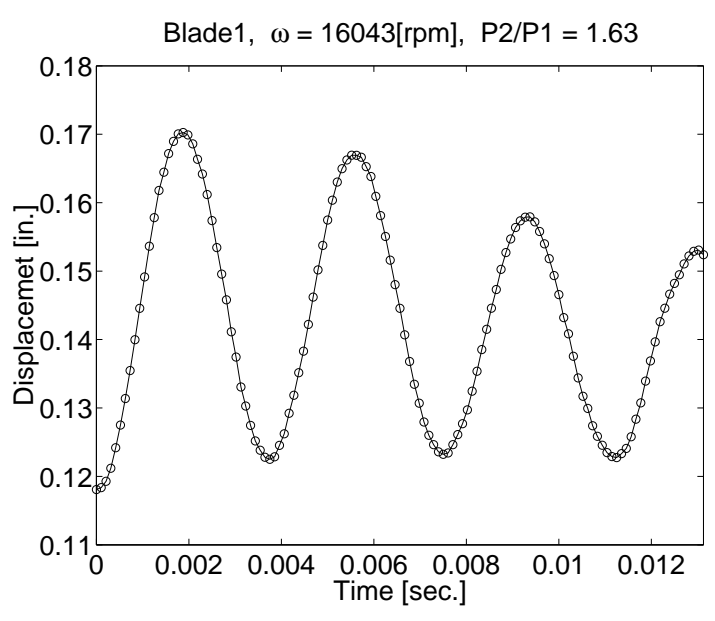

(b) $\sigma=270$ deg.

Figure 4.18: Deflection at the Mid-Chord of the Tip Section for Near Peak Efficiency, $\sigma=90,270 \mathrm{deg}$

response. These results implies that the inter-blade phase angle which produces the least stable response may exist near $\sigma=0$ degrees.

To examine how the unsteady pressure influences the stability of the blade motion, the unsteady pressure may be decomposed by a Fourier analysis into the components contributing and not contributing to the damping. Figure 4.19 shows the calculated distribution of the first harmonic imaginary unsteady pressure along with the distribution of the mean value of the unsteady pressure on the suction and pressure sides, respectively. The results are presented for $\sigma=0$ degrees. The mean surface pressures in Figure 4.19 are obtained by averaging the unsteady pressures over the last oscillation cycle. The first harmonic imaginary components of the pressure perturbations are supposed to act in accordance with damping terms.

As shown, significant unsteady pressure amplitudes exist in the region where the shocks sit on the blade. On the suction side, the shock appears along the entire span as a continuous line. However, the imaginary pressure distribution shows the peak along 


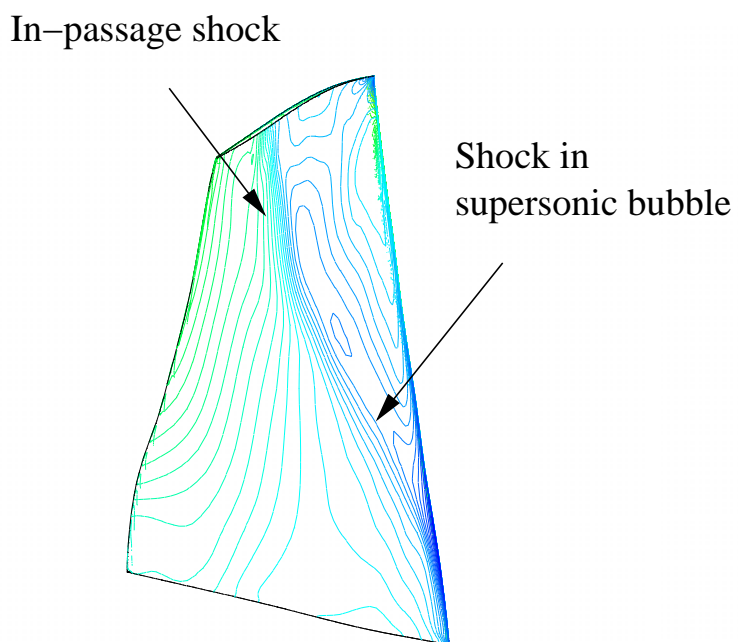

(a) Mean Value - Suction Side

In-passage shock

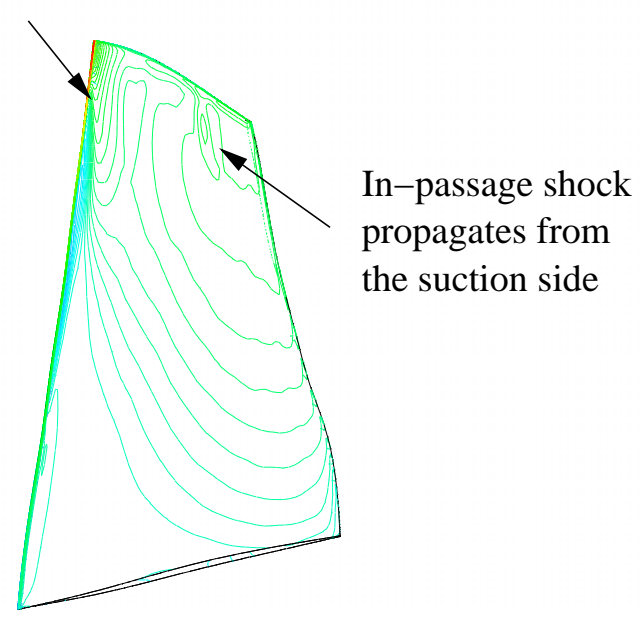

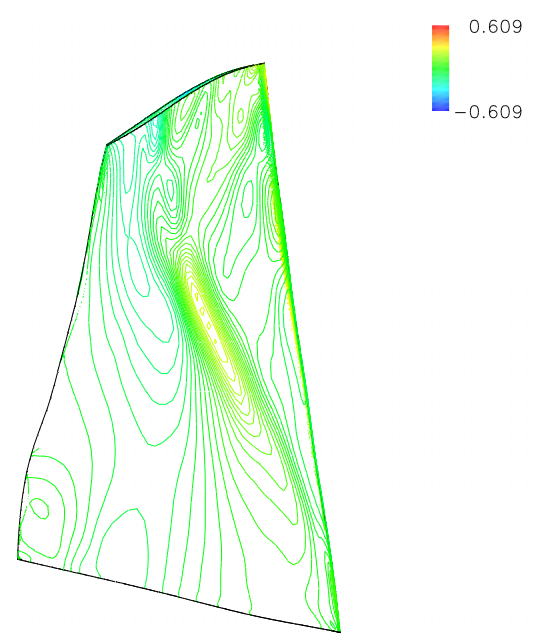

(b) 1st Harmonic Imaginary Value -Sucsion Side

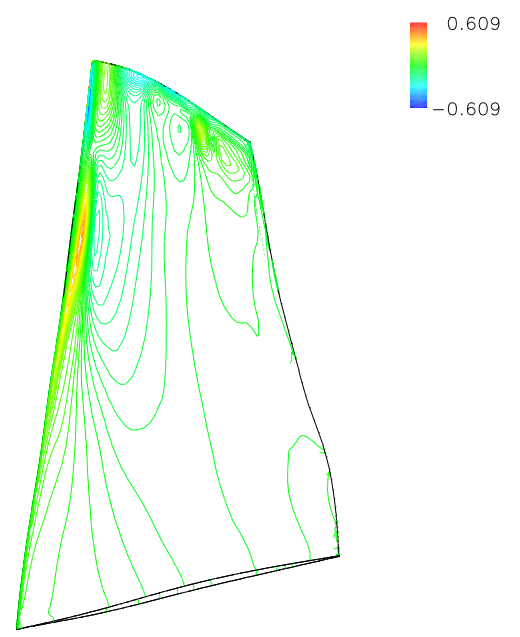

(c) Mean Value - Pressure Side

(d) 1st Harmonic Imaginary Value -Pressure Side

Figure 4.19: Unsteady Pressure Distribution on the Blade Surfaces for Near Peak Efficiency, $\sigma=0 \mathrm{deg}$ 
the shock is split into two parts. Between the casing and 40 percent of the span, the passage shock is very strong and indicates that an adverse pressure gradient follows that may cause shock induced separation. On the other hand, below 40 percent of span, the shock is formed inside the supersonic bubble and does not reach the pressure side of the adjacent blade as seen in the Mach contour at the 70 percent span location in Figure 4.13. These two kinds of shocks behave differently as damping forces. While the in-passage shock generates a negative peak in the imaginary pressure distribution, the shock in the supersonic bubble generates a positive peak. On the pressure side, the imaginary pressure distribution also indicates peaks along the in-passage shock near the leading edge. In addition, it is shown that the in-passage shock on the suction side propagates over the tip clearance to the pressure side of the blade around the middle chord near the tip. This small shock generates another peak in the unsteady pressure as well.

Figure 4.20 shows the mean and first harmonic imaginary pressure distributions for $\sigma=180$ degrees. The results show that considerably larger unsteady forces arise when the motion of adjacent blades is out of phase. For this case, the two kinds of shocks on the suction side mentioned above do not form a continuous line, and the oblique shock from the leading edge of the adjacent blade strikes more prominently near the tip on the suction side. In particular, the in-passage shock on the suction side shows a totally different peak of the unsteady pressure, although the other shocks on the suction side do not generate peaks.

Now that it has been shown that the shock oscillations generate large pressure fluctuations, the next question will be whether these fluctuations damp or excite the blade motion. To examine this, the work per cycle is a parameter often used to represent the contributions of unsteady pressure fields to the stability of the blade motion. The work per cycle, $W_{C}$, is the work done by the fluid on a given blade over one cycle of its motion. It is obtained by computing the product of the pressure force 
In-passage shock

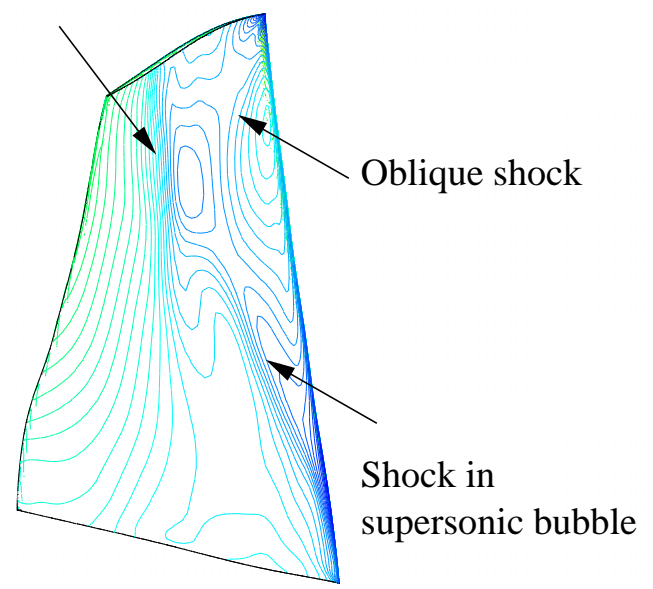

(a) Mean Value - Suction Side

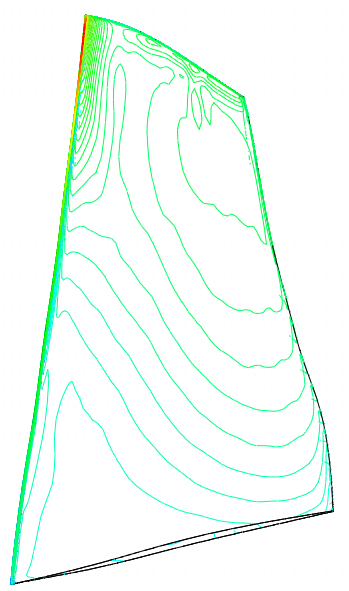

(c) Mean Value - Pressure Side

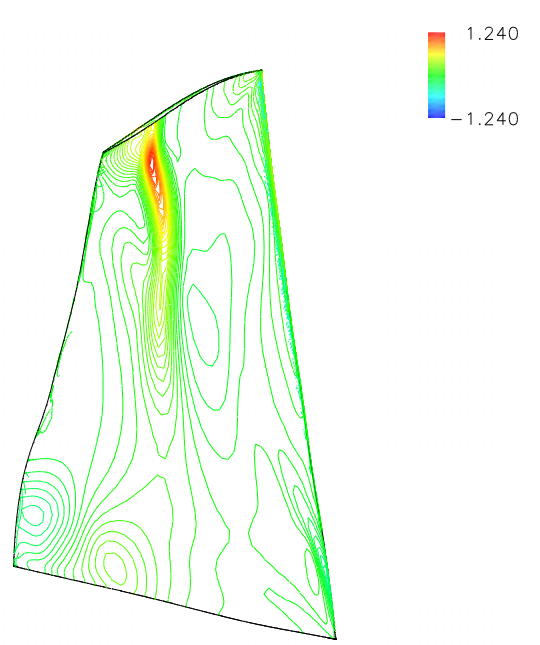

(b) 1st Harmonic Imaginary Value -Sucsion Side

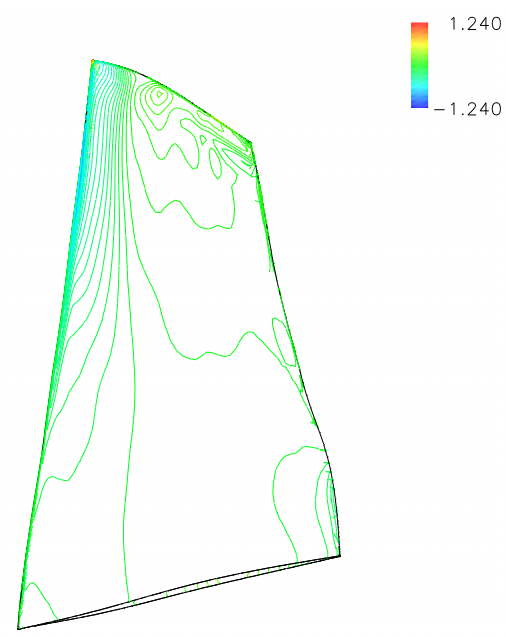

(d) 1st Harmonic Imaginary Value -Pressure Side

Figure 4.20: Unsteady Pressure Distribution on the Pressure Side for Near Peak Efficiency, $\sigma=180 \mathrm{deg}$ 
and the associated differential displacement.

$$
W_{C}=\int_{t}^{t+T} \int_{\partial \Omega} p(x, y, z, t) \dot{\boldsymbol{q}}(x, y, z, t) \cdot d \boldsymbol{S} d t
$$

In this equation, negative work implies positive damping in which aerodynamic forces act in the opposite direction of the velocity of the displacements. Therefore, a blade motion is stable, neutrally stable, or unstable according to whether the work per cycle is less than, equal to, or greater than zero, respectively. Figures 4.21 and 4.22 show the aerodynamic work per cycle of the last period for $\sigma=0$ and 180 degrees, respectively. For $\sigma=0$ degrees, a negative peaks appear at the locations where the oblique shock and the lower part of the shock sit on the suction side. Therefore, the oblique shock and the lower shock oscillations work to dampen the blade motion. On the pressure side, there is a positive peak near the location of the in-passage shock. Notice that the regions of exciting and damping forces on both sides are quite close. That means that the exciting and damping forces generated by the shock oscillations cancel each other resulting in an almost constant amplitude motion. On the other hand, the distributions for $\sigma=180$ degrees show that the forces generated by the in-passage shock on the suction side works to dampen the oscillation, while the peaks along the oblique shock and the lower part of the shock disappear. For this case almost the entire suction side is associated with damping forces. Is is also obvious that the in-passage shock on the pressure side produces a large damping force toward the leading edge. Thus the physical mechanism for the decaying response of the $\sigma$ $=180$ case is clearly explained. It is also implied in this discussion that the phase change of the imaginary part of the unsteady pressure is associated with stabilization or destabilization of the oscillation.

In order to validate the arguments developed so far, two other operation points were chosen to examine the effect of a different kind of shock structure. One is a near 


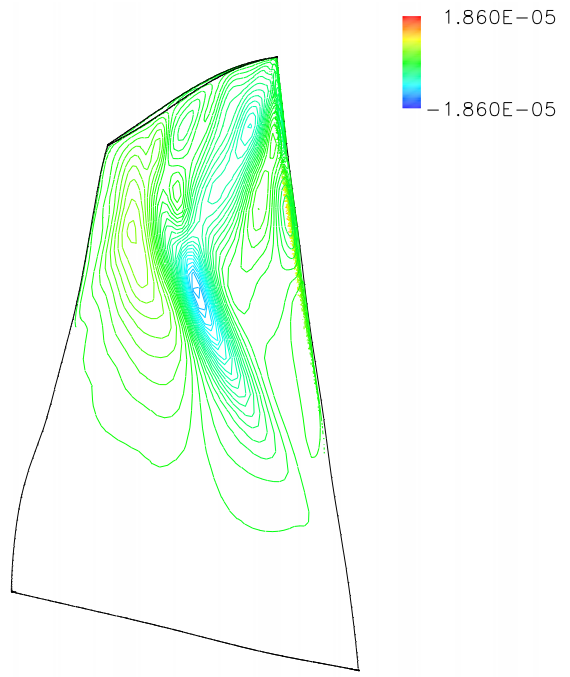

(a) Suction Side

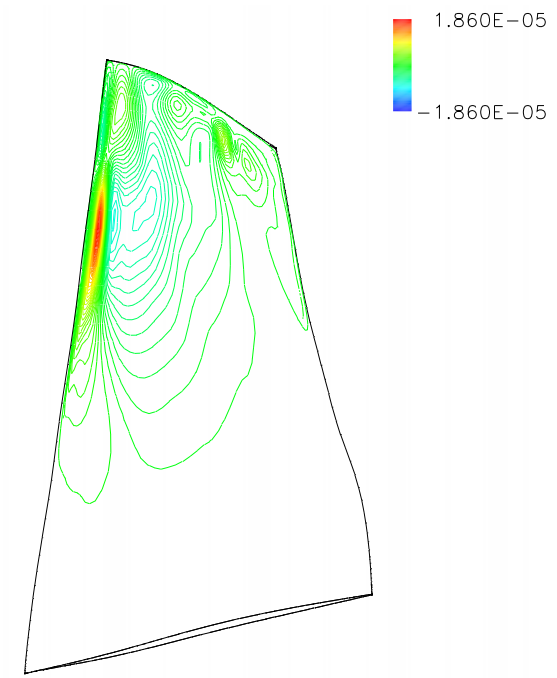

(b) Pressure Side

Figure 4.21: Work per Cycle Distribution on the Blade Surface for Near Peak Efficiency, $\sigma=0 \mathrm{deg}$ 


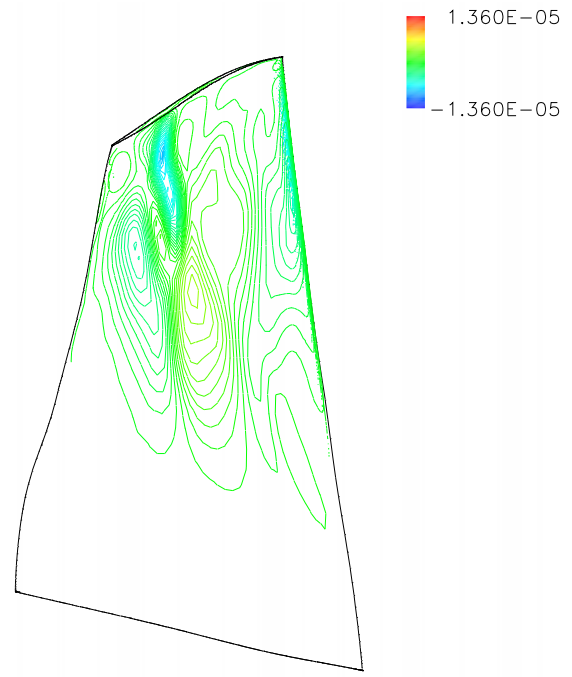

(a) Suction Side

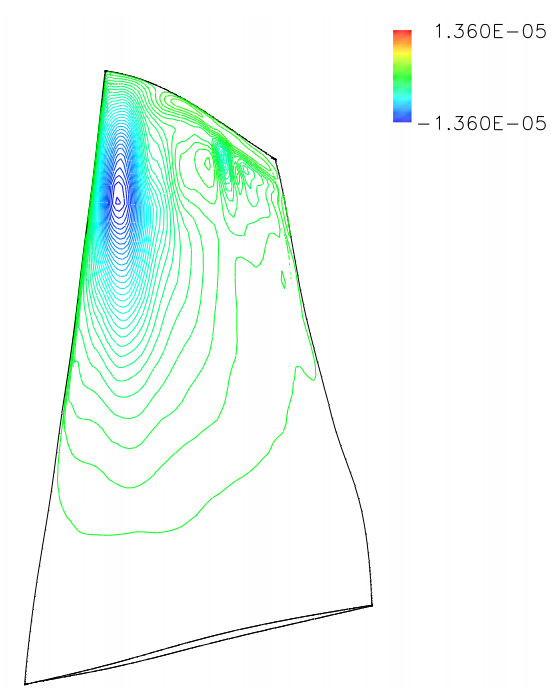

(b) Pressure Side

Figure 4.22: Work per Cycle Distribution on the Blade Surface for Near Peak Efficiency, $\sigma=180 \mathrm{deg}$ 


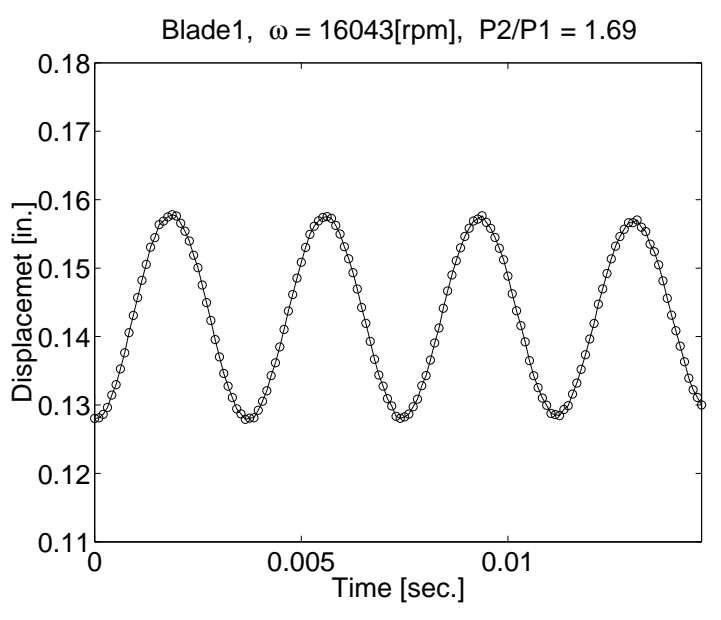

(a) $\sigma=0$ deg.

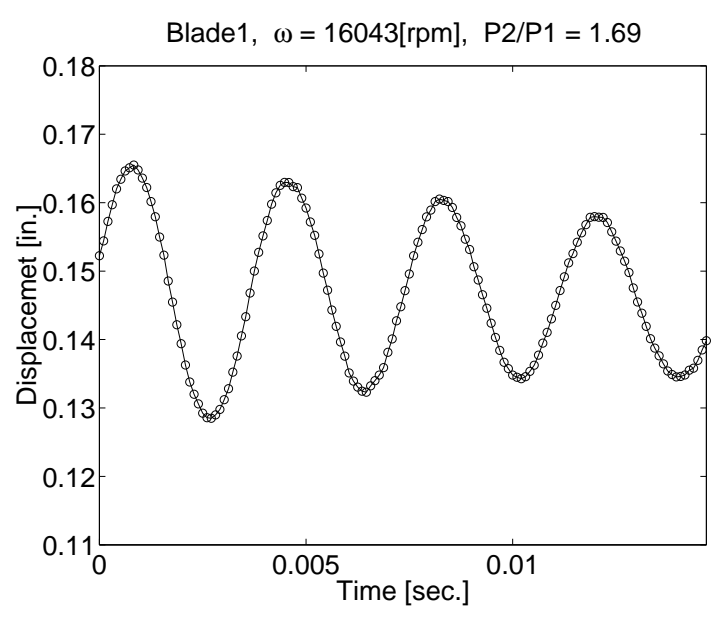

(b) $\sigma=180 \mathrm{deg}$.

Figure 4.23: Deflection at the Mid-Chord of the Tip Section for Near Stall, $\sigma=$ $0,180 \mathrm{deg}$

stall operating point corresponding to a total pressure ratio of 1.69 , and the other is a near choke operating point corresponding to a total pressure ratio of 1.5.

As shown in Section 4.2.4, a detached bow shock is formed near the tip and the pressure side is shock-free at the near stall operating point. Figure 4.23 shows the aeroelastic responses for $\sigma=0$ and 180 degrees at the near stall point. Similarly to the near peak efficiency point, while the response for $\sigma=0$ degrees shows an oscillation with an almost constant amplitude, the response for $\sigma=180$ degree shows a decaying oscillation. Again, the aerodynamic damping for $\sigma=180$ degrees is greater than that for $\sigma=0$ degrees.

In order to investigate the locations of strong damping effects, the work per cycle was calculated for these cases too. Figure 4.24 shows the work per cycle distributions for the near stall operating condition cases. A similar trend to the near peak efficiency case can be seen on the suction surface. While the lower shock stabilizes the motion, the bow shock sitting near the tip region excites the blade motion. Since the shock 
wave near the tip region doesn't sit on the pressure side for the near stall case, no major peak in the work per cycle can be seen on the pressure surface.

Figure 4.25 shows the Mach contours for the near choke case. The in-passage shock is much stronger and extends from the middle of the pressure side to the trailing edge on the suction side. The oblique shock generated at the leading edge near the tip region reaches the suction side of the adjacent blade. Even at the 70 percent span station, not only the supersonic bubble, but also the shock near the middle of the chord can be observed.

Figure 4.27 shows the work per cycle distributions for the near choke case. From the picture of the suction side for $\sigma=0$ degrees, it is implied that the shock inside the supersonic bubble near the hub has developed to form the oblique shock toward the tip region. The oblique shock also reaches the pressure side of the adjacent blade. Similarly to the near peak efficiency cases, the in-passage shock produces an excitation force on the upper part of the suction surface for $\sigma=0$ degree case. On the other hand, the pressure surface experiences damping forces from the inpassage shock which cancel the exciting force on the suction side. When the cascade has an inter-blade phase angle of 180 degrees, the peak on the suction side due to the in-passage shock disappears. Although exciting forces appear on the pressure side instead, the damping forces due to the in-passage shock and the oblique shock dominate the stability. 


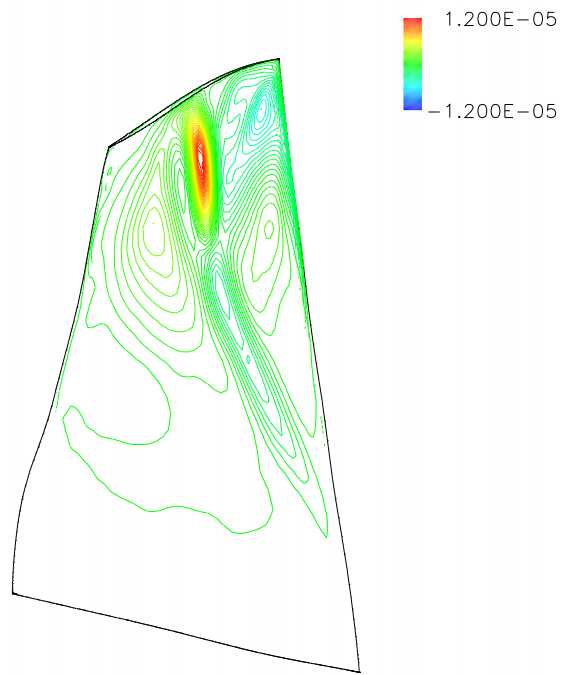

(a) Suction Side - $\sigma=0$ deg.

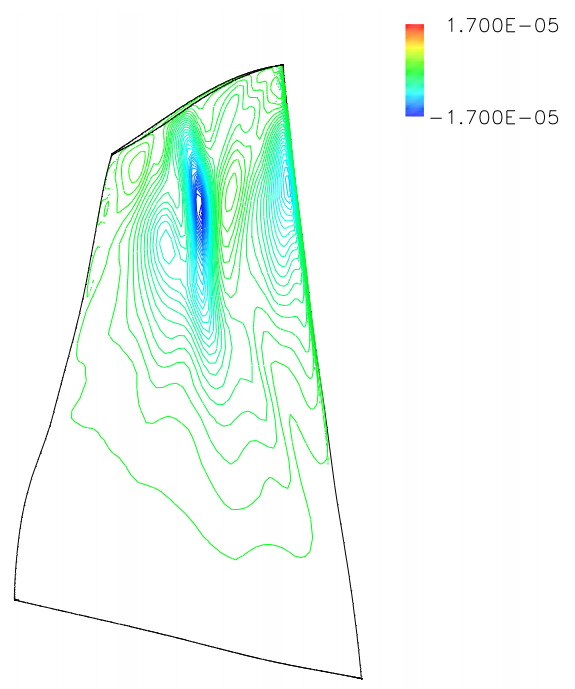

(c) Suction Side $-\sigma=180$ deg.

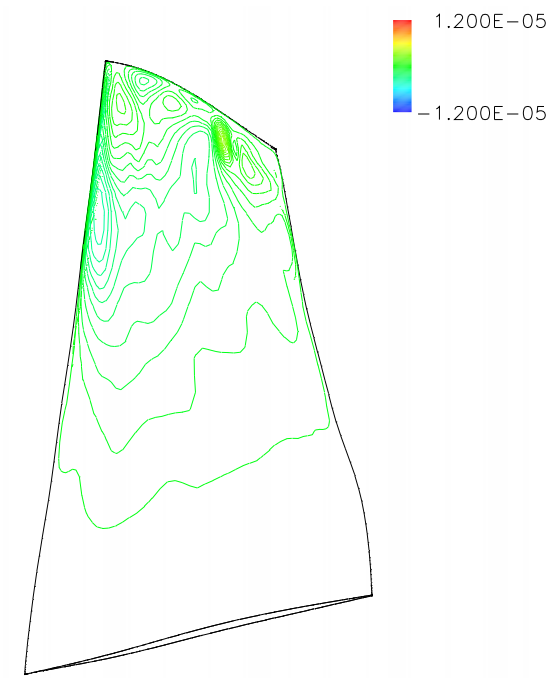

(b) Pressure Side $-\sigma=0$ deg.

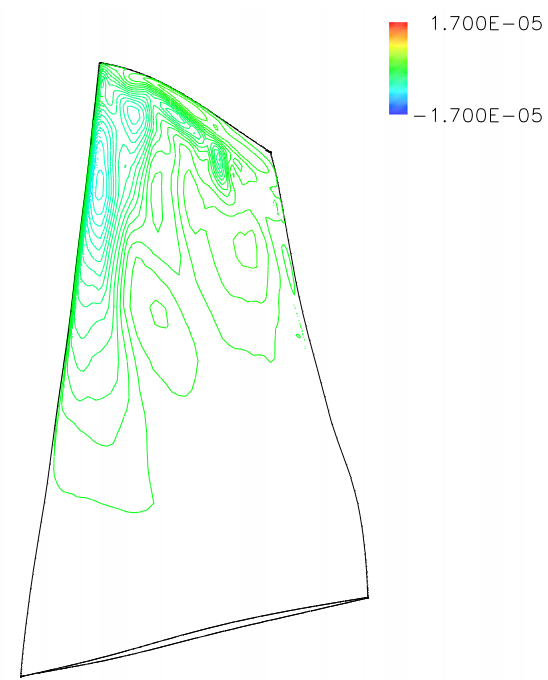

(d) Pressure Side $-\sigma=180 \mathrm{deg}$.

Figure 4.24: Work per Cycle Distribution on the Blade Surface for Near Stall, $\sigma=$ $0,180 \mathrm{deg}$ 


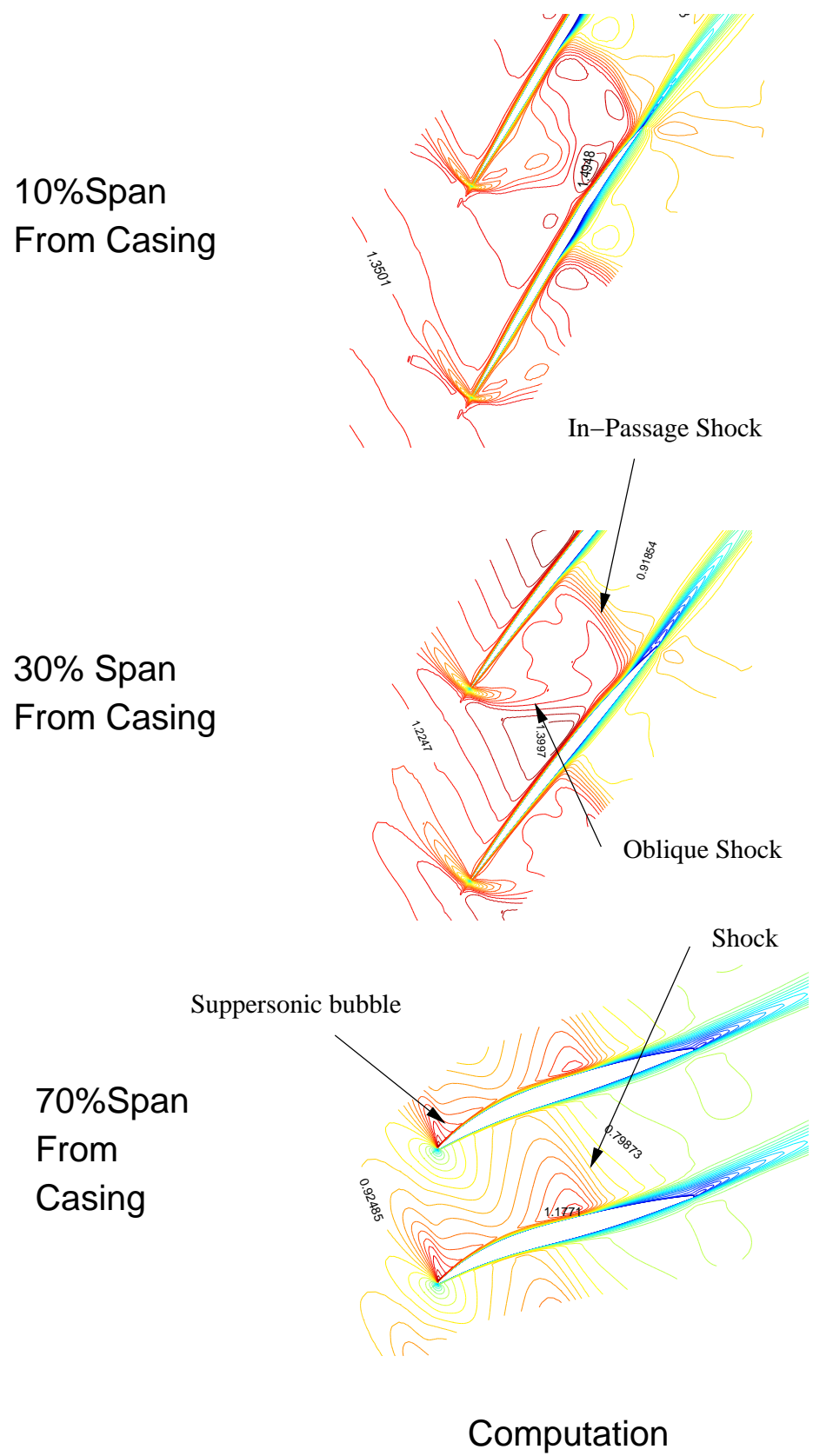

Figure 4.25: Experimental and Numerical Relative Mach Number Contour for Near Choke 


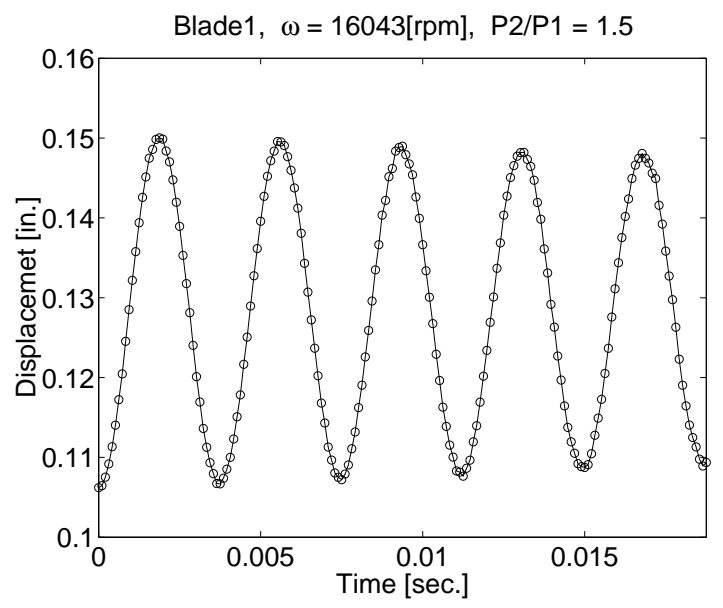

(a) $\sigma=0$ deg.

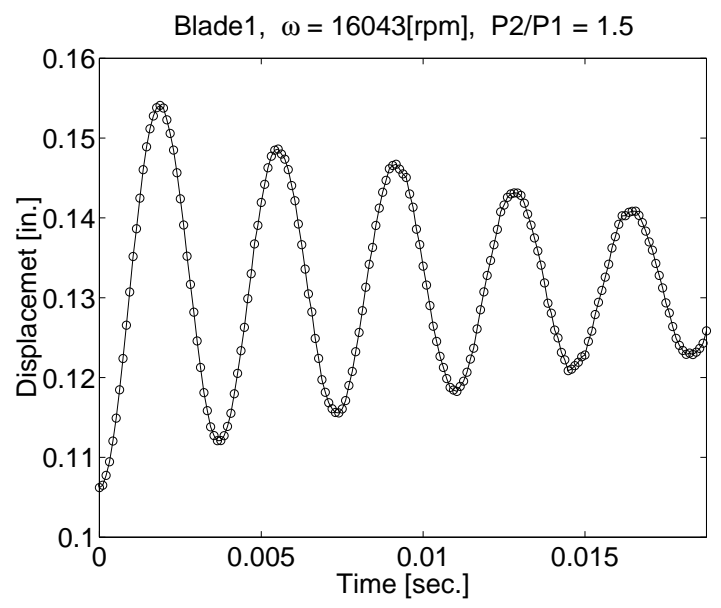

(b) $\sigma=180$ deg.

Figure 4.26: Deflection at the Mid-chord of the Tip Section for Near Choke, $\sigma=$ $0,180 \mathrm{deg}$ 
In-passage shock

Peak along the in-passage shock

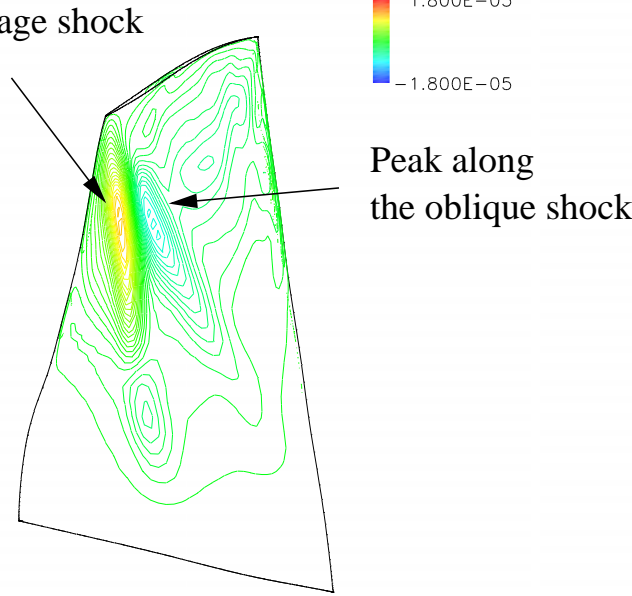

(a) Suction Side -0 deg.

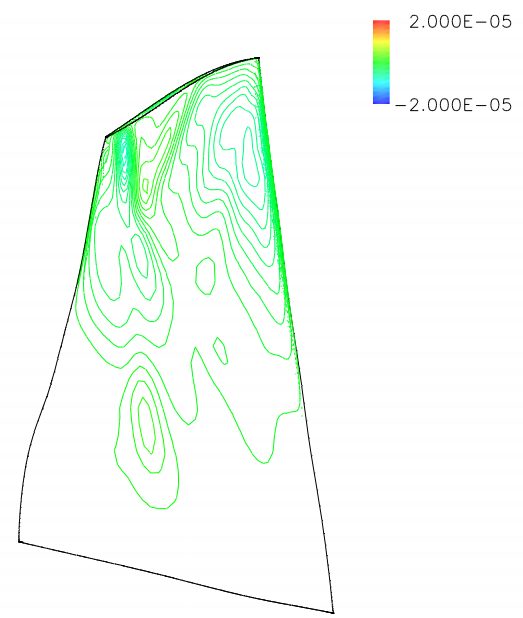

(c) Suction Side - $180 \mathrm{deg}$.

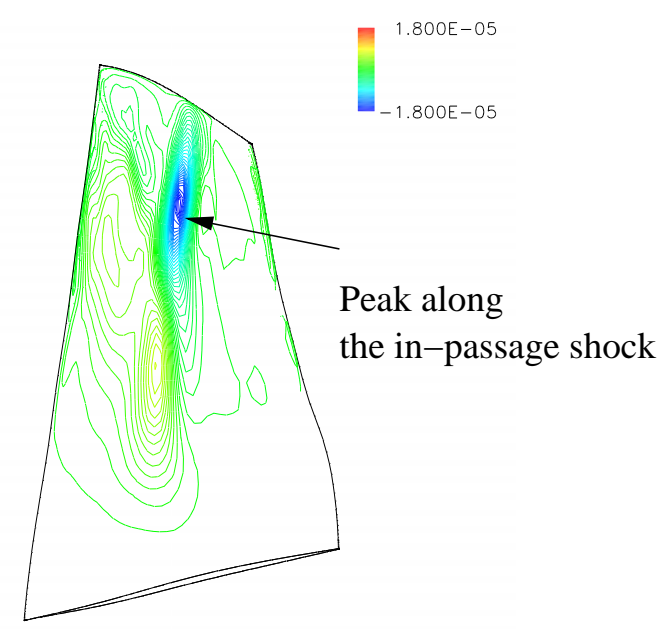

(b) Pressure Side - 0 deg.

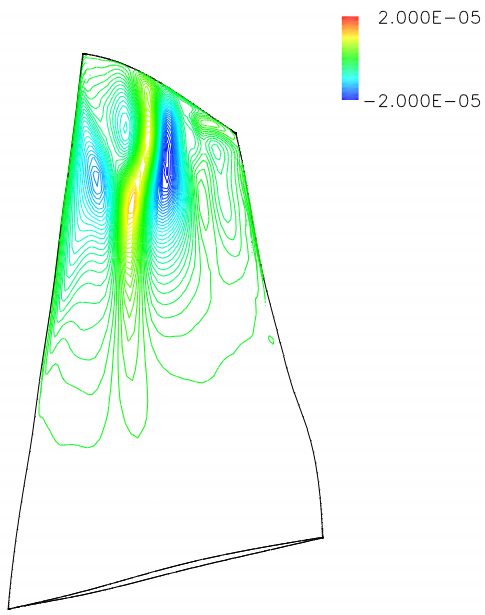

(d) Pressure Side - 180 deg.

Figure 4.27: Work per Cycle Distribution on the Blade Surface for Near Choke, $\sigma=0,180 \mathrm{deg}$ 


\section{Chapter 5}

\section{Conclusions and Future Work}

A fluid/structure coupled aeroelastic solver for turbomachinery based on a threedimensional unsteady RANS solver, TFLO, and the finite element structural analysis package, MSC/NASTRAN, was developed for use in turbomachinery flutter simulations. The capabilities of the solver are demonstrated by applying it to investigate the forced response characteristics of a transonic fan rotor, NASA Rotor 67. The aeroelasitic solver successfully produces decaying or growing responses of the structural motion for turbomacninery depending on the flow conditions, i.e. operating points and inter-blade phase angles. Calculations were also carried out for an isolated wing in transonic flow.

Results presented for Rotor 67 revealed that the main contribution to the stability of a transonic fan is in the unsteady forces generated by the shock motions. In determining these shock motions, inter-blade phase angles play the most important role. The phase angle between the blade motion and the shock motion determines whether the unsteady forces behave to damp or excite the oscillations. For detecting the source of the exciting forces, work per cycle distributions on the blade surfaces are very useful. One of the greatest benefits of a fluid/structure coupling solution procedure is found to be the ability to visualize the phenomena, including the blade 
motion, which can aid in developing a methodology to avoid the instabilities by modifying the blade shape or its structure.

It should be noted that difficulties in obtaining an unsteady aeroelastic response are often associated with the calculation of the steady-state flow. The lessons learned here are that if difficulties in convergence are encountered in the unsteady calculations, the initial steady-state solution would be suspicious. It might be a solution in the middle of a steady-state calculation which would eventually diverge. A proper level of convergence for the initial steady-state solution is one of the most important factors for a successful unsteady computation in which a large amount of iterations follow the computation for obtaining the initial condition. In order to obtain a converged initial solution, a proper choice of turbulence model and an accurate treatment of the geometry of the tip clearance region are important in turbomachinery computations. In the tip clearance region, the mixture of extremely nonlinear transonic flow and heavy shear flow characteristics causes rapid variations in solutions which would require the proper modelings of the tip clearance for stable computations. Furthermore, these modelings would affect the stability in unsteady computations because the mesh in this region is the most sensitive in keeping its original quality during mesh perturbations as mentioned in Section 3.1.8.

One of the biggest concerns for these fluid/structure computations is that the coupled procedure is still computationally expensive. The prediction of flutter boundaries with approaches such as time-linearized methods may be useful for single blade rows. Advanced engines, however, will likely require aeroelastic analysis for multiple blade rows, or a whole wheel system to admit all possible frequencies and inter-blade phase angles. Fluid/structure couplings such as the one presented here would contribute to these kinds of numerical predictions for aeroelastically severe conditions. In addition, numerical analysis of aeroelastically related phenomena in turbomachinery such as flutter, rotating stall, inlet distortion, mistunings, etc. will be less expensive than the 
experimental validations. Furthermore, one of the biggest benefits of the numerical analysis is its richness in data that the experiment has been lacking due the difficulties in installing the measurement instruments.

Whole system analyses will be required for making reliable blade and aeroelastic response predictions. As far as the structural modeling is concerned, such analyses must include multi-component coupling, such as blade-disk and disk-rotor interactions and support system flexibilities. Mechanical damping in turbomachines occurs during rubbing at mating interfaces: shroud, root, blade-to-blade or blade-to-disk dampers. More accurate modeling of mechanical damping will be needed for making better aeroelastic predictions. In addition, the modeling of gyroscopic terms will be a problem that should be solved. 


\section{Bibliography}

[1] E. H. Dowell, H. C. Curtiss, R. H. Scanlan, and F. Sisto. A Modern Course in Aeroelasticity, chapter 8, pages 411-442. Kluwer Academic Publishers, 1989.

[2] F. Sisto. A Survey of Current Problems in Turbomachine Aeroelasticity. In Recent Trends in Aeroelasticity, Structures, and Structural Dynamics, pages 48-62. University Press of Florida, 1986.

[3] E. Szechenyi. Understanding Fan Blade Flutter Through Linear Cascade. In AGARD Manual on Aeroelasticity in Axial-Flow Turbomachines, number AGARDograph No. 298, chapter 1-10. 1987.

[4] D. G. Halliwell. Effect of Intake Conditions on Supersonic Unstalled Flutter in Turbofan Engines. Journal of Aircraft, 17(5):300-304, May 1980.

[5] O. O. Bendiksen. Role of Shocks in Transonic/Supersonic Compressor Rotor Flutter. AIAA Journal, 24(7):1179-1186, July 1986.

[6] H. Stargardter. Fan Flutter Test. In AGARD Manual on Aeroelasticity in AxialFlow Turbomachines, number AGARDograph No. 298, chapter 2-10. 1987.

[7] D. S. Whitehead. Classical Two-Dimensional Methods. In AGARD Manual on Aeroelasticity in Axial-Flow Turbomachines, number AGARDograph No. 298, chapter 1-3. AGARD, 1987.

[8] O. O. Bendiksen. Aeroelastic Problems in Turbomachines. In Flight-Vehicle Materials, Structures, and Dynamics, volume 5 of 2, chapter 5, pages 241-297. ASME, 1993. 
[9] J. M. Verdon. Review of Unsteady Aerodynamic Method for Turbomachinery Aeroelastic and Aeroacoustic Applications. AIAA Journal, 31(2):235-250, February 1993.

[10] F. O. Carta. Coupled Blade-Disk-Shroud Flutter Instabilities in Turbojet Engine Rotors. Journal of Engineering for Power, 89(3):419-426, July 1967.

[11] D. S. Whitehead. Force and Moment Coefficients for Vibrating Aerofoils in Cascade. Technical Report Reports and Memoranda 3254, Aeronautical Research Council, February 1960.

[12] D. S. Whitehead. Bending Flutter of Unstalled Cascade Blades at Finite Deflection. Technical Report Reports and Memoranda 3386, Aeronautical Research Council, October 1962.

[13] H. Atassi and T. J. Akai. Aerodynamic and Aeroelastic Characteristics of Oscillating Loaded Cascades at Low Mach Number Part 1: Pressure Distribution, Forces, and Moments. Journal of Engineering for Power, 102(2):344-351, April 1980.

[14] T. J. Akai and H. Atassi. Aerodynamic and Aeroelastic Characteristics of Oscillating Loaded Cascades at Low Mach Number Part 2: Stability and Flutter Boundary. Journal of Engineering for Power, 102(2):352-356, April 1980.

[15] F. Lane and M. Friedman. Theoretical Investigation of Subsonic Oscillatory Blade-Row Aerodynamics. Technical Report NACA-TN-4136, February 1958.

[16] S. N. Smith. Discrete Frequency Sound Generation in Axial Flow Turbomachines. Technical Report Reports and Memoranda 3709, Aeronautical Research Council, March 1972.

[17] M. Namba. Subsonic Cascade Flutter with Finite Mean Lift. AIAA Journal, 13(5):586-593, May 1975.

[18] J. M. Verdon and J. E. McCune. Unsteady Supersonic Cascade in Subsonic Axial Flow. AIAA Journal, 13(2):193-201, February 1975. 
[19] T. Nagashima and D. S. Whitehead. Linearized Supersonic Unsteady Flow in Cascades. Technical Report Reports and Memoranda 3811, Aeronautical Research Council, February 1977.

[20] M. E. Goldstein, W. Braun, and J. J. Adamczyk. Unsteady Flow in a Supersonic Cascade with Strong In-Passage Shocks. Journal of Fluid Mechanics, 83(3):569604, December 1977.

[21] M. Namba and A. Ishikawa. Three-Dimensional Aerodynamic Characteristic of Oscillating Supersonic and Transonic Annular Cascades. Journal of Engineering for Power, 105(2):138-146, January 1983.

[22] J. M. Verdon and J. R. Casper. A Linearized Unsteady Aerodynamic Analysis for Transonic Cascades. Journal of Fluid Mechanics, 149:403-429, 1984.

[23] D. S. Whitehead. A Finite Element Solution of Unsteady Two-Dimensional Flow in Cascades. International Journal for Numerical Methods in Fluids, 10:13-34, 1990.

[24] K. C. Hall and E. F. Crawley. Calculation of Unsteady Flows in Turbomachinery Using Linearized Euler Equations. AIAA Journal, 27(6):777-787, June 1989.

[25] K. C. Hall and C. B. Lorence. Calculation of Three-Dimensional Unsteady Flows in Turbomachinery Using the Linearized Harmonic Euler Equations. Journal of Turbomachinery, 115(4):800-809, October 1993. ASME-92-GT-136.

[26] W. Ning and L. He. Computation of Unsteady Flows Around Oscillating Blades Using Linear and Nonlinear Harmonic Euler Methods. Journal of Turbomachinery, 120(3):508-514, July 1998. ASME-97-GT-229.

[27] R. H. Ni and F. Sisto. Numerical Computation of Nonstationary Aerodynamics of Flat Plate Cascades in Compressible Flow. Journal of Engineering for Power, 98(2):165-170, April 1976. 
[28] T. H. Fransson and M. Pandolfi. Numerical Investigation of Unsteady Subsonic Compressible Flows through an Oscillating Cascade. In ASME 31st International Gas Turbine Conference and Exhibit, June 1986.

[29] G. A. Gerolymos. Numerical Integration of the Blade-to-Blade Surface Euler Equations in Vibrating Cascades. AIAA Journal, 26(12):1483-1492, December 1988.

[30] L. He. An Euler Solution for Unsteady Flows Around Oscillating Blades. Journal of Turbomachinery, 115(4):714-722, October 1990. ASME-89-GT-279.

[31] J. I. Erdos, E. Alzer, and W. McNally. Numerical Solution of Periodic Transonic Flow through a Fan Stage. AIAA Journal, 15(11):1559-1568, November 1975.

[32] G. A. Gerolymos. Advances in the Numerical Integration of the ThreeDimensional Euler Equations in Vibrating Cascades. Journal of Turbomachinery, 115(4):781-790, October 1993. ASME-92-GT-170.

[33] D. Peitsch, H. E. Gallus, and S. Weber. Computation of Unsteady Transonic 3DFlow in Turbomachine Bladings. In Unsteady Aerodynamics and Aeroelasticity of Turbomachines, pages 161-176. Elsevier, 1995.

[34] R. W. MacCormack. Numerical computation of compressible viscous flow. Course Reader of AA214 Stanford University, 1995.

[35] D. L. Huff. Numerical Simulations of Unsteady, Viscous, Transonic Flow Over Isolated and Cascaded Airfoils Using a Deforming Grid. In AIAA 19th Fluid Dynamics, Plasma Dynamics and Lasers Conference, number AIAA-87-1316, June 1987.

[36] L. D. G. Siden. Numerical simulation of unsteady viscous compressible flows applied to blade flutter analysis. In ASME 36th International Gas Turbine and Aeroengine Congress and Exposition, number ASME-91-GT-203, June 1991. 
[37] L. He and J. D. Denton. Inviscid-Viscous Coupled Solution for Unsteady Flows Through Vibrating Blades: Part 1 - Description of the Method. Journal of Turbomachinery, 115(1):94-100, January 1993. ASME-91-GT-125.

[38] L. He and J. D. Denton. Inviscid-Viscous Coupled Solution for Unsteady Flows Through Vibrating Blades: Part 2 - Computational Results. Journal of Turbomachinery, 115(1):101-109, January 1993. ASME-91-GT-126.

[39] S. Ji and F. Liu. Flutter Computation of Turbomachinery Cascades Using a Parallel Unsteady Navier-Stokes Code. AIAA Journal, 37(3):320-327, March 1999.

[40] L. He and J. D. Denton. Three-Dimensional Time-Marching Inviscid and Viscous Solutions for Unsteady Flows Around Vibrating Blades. Journal of Turbomachinery, 116(3):469-476, July 1994. ASME-93-GT-92.

[41] A. J. Strazisar, J. R. Wood, M. D. Hathaway, and K. L. Suder. Laser Anemometer Measurements in a Transonic Axial-Flow Fan Rotor. Technical Report NACA-TP-2879, 1989.

[42] M. A. Bakhle, R. Srivastava, Jr. T. G. Keith, and G. L. Stefko. A 3D Euler/Navier-Stokes Aeroelastic Code for Propulsion Applications. In AIAA 33rd AIAA/ASME/SAE/ASEE Joint Propulsion Conference and Exhibit, number AIAA-97-2749, July 1997.

[43] B. S. Baldwin and H. Lomax. Thin Layer Approximation and Algebraic Model for Separated Turbulent Flows. In AIAA 16th Aerospace Sciences Meeting, number AIAA-78-257, January 1978.

[44] P. A. Storey. Holographic Vibration Measurement of a Rotating Fluttering Fan. In Proceedings of AIAA/SAE/ASME 18th Joint Propulsion Conference, number AIAA-82-1271, June 1982.

[45] D. S. Whitehead. Torsional Flutter of Unstalled Cascade Blades at Zero Deflection. Technical Report Reports and Memoranda 3429, Aeronautical Research Council, March 1964. 
[46] O. O. Bendiksen and P. Friedmann. Coupled Bending Torsion Flutter in Cascades. AIAA Journal, 18(2):194-201, February 1980.

[47] R. L. Bispringhoff, H. Ashley, and R. L. Halfman. Aeroelasticity. AddisonWesley, 1955.

[48] K. Rao, V. Kaza, and R. E. Kielb. Flutter of Turbofan Rotor with Mistuned Blades. AIAA Journal, 22(11):1618-1625, November 1984.

[49] G. A. Gerolymos. Coupled Three-Dimensional Aeroelastic Stability Analysis of Bladed Disks. Journal of Turbomachinery, 115(4):791-799, October 1993. ASME-92-GT-171.

[50] T. S. R. Reddy, M. A. Bakhle, D. L. Huff, and T. W. Swafford. Analysis of Cascades Using a Two-Dimensional Euler Aeroelastic Solver. In Proceedings of AIAA/ASME/ASCE/AHS/ASC 33rd Structures, Structural Dynamics and Material Conference, number AIAA-92-2370, April 1992.

[51] L. He. Rotating-Stall/Stall-Flutter prediction Using A Fluid/Structure Coupled Method. In Unsteady Aerodynamics and Aeroelasticity of Turbomachines, pages 597-607. Elsevier, 1995.

[52] M. Vahdati and M. Imregun. Nonlinear Aeroelasticity Analysis Using Unstructured Dynamics Meshes. In Unsteady Aerodynamics and Aeroelasticity of Turbomachines, pages 177-196. Elsevier, 1995.

[53] J. W. Chew, J. G. Marshall, M. Vahdati, and M. Imregun. Part-Speed Flutter Analysis of a Wide-Chord Fan Blade. In Unsteady Aerodynamics and Aeroelasticity of Turbomachines, pages 707-724. Kluwer Academic Publisher, 1998.

[54] D. A. Gottfried and S. Fleeter. Aerodynamic Damping Predictions for Turbomachine Blade Rows Using a Three-Dimensional Time Marching Simulation. In AIAA/ASME/SAE/ASEE Joint Propulsion Conference and Exhibit, number AIAA-99-2810, June 1999. 
[55] H. Doi and J. J. Alonso. Fluid/Structure Coupled Aeroelastic Computations for Transonic Flows in Turbomachinery. In ASME Turbo Expo 2002, number GT-2002-30313, June 2002.

[56] J. Yao, A. Jameson, J. J. Alonso, and F. Liu. Development and Validation of a Massively Parallel Flow Solver for Turbomachinery Flows. In 38th Aerospace Sciences Meeting and Exhibit, number AIAA-00-0882, June 2000.

[57] J. Yao, R. L. Davis, J. J. Alonso, and A. Jameson. Unsteady Flow Investigations in an Axial Turbine Using the Massively Parallel Flow Solver TFLO. In 39th Aerospace Sciences Meeting and Exhibit, number AIAA-2001-0529, June 2001.

[58] J. Yao, A. Jameson, J. J. Alonso, and F. Liu. Development and Validation of a Massively Parallel Flow Solver for Turbomachinery Flows. Journal of Propulsion and Power, 17(3):659-668, May 2001.

[59] R. L. Davis, J. Yao, J. P. Clark, G. Stetson J. J. Alonso, A. Jameson, C. W. Haldeman, and M. G. Dunn. Unsteady Interaction Between a Transonic Turbine Stage and Downstream Components. In ASME Turbo Expo 2002, number GT2002-30364, June 2002.

[60] M. W. Rubesin and W. C. Rose. The Turbulent Mean-Flow, Reynolds-Stress, and Heat Flux Equations in Mass-Averaged Dependent Variables. Technical Report NASA-TM-X-62248, March 1973.

[61] P. D. Thomas and C. K. Lombard. Geometric Conservation Law and Its Application to Flow Computations on Moving Grids. AIAA Journal, 17(10):10301037, October 1979.

[62] C. Farhat, M. Lesoinne, and N. Maman. Mixed Explicit/Implicit Time Integration of Coupled Aeroelastic Problems: Three-Field Formation, Geometric Conservation and Distributed Solution. International Journal for Numerical Methods in Engineering, 21:807-835, 1995. 
[63] A. Jameson, W. Schmidt, and E. Turkel. Numerical Solutions of Euler Equations by Finite Volume Method with Runge-Kutta Time Stepping Scheme. In AIAA 14th Fluid and Plasma Dynamics Conference, Palo Alto, number AIAA81-1259, June 1981.

[64] A. Harten. High Resolution Schemes for Hyperbolic Conservation Laws. Journal of Computational Physics, 49(3):357-393, March 1983.

[65] A. Jameson. Analysis and Design of Numerical Schemes for Gas Dynamics 1 - Artificial Diffusion, Upwind Biasing, Limiters and Their Effect on Accuracy and Multigrid Convergence. International Journal of Computational Dynamics, 4:171-218, 1995.

[66] A. Jameson. Analysis and Design of Numerical Schemes for Gas Dynamics 2 - Artificial Diffusion and Discrete Shock Structure. International Journal of Computational Dynamics, 5:1-38, 1995.

[67] A. Jameson. Time Dependant Calculations Using Multigrid, with Applications to Unsteady Flows Past Airfoils and Wings. In Proceedings of AIAA 10th Computational Fluid Dynamics Conference, number AIAA-91-1596, June 1991.

[68] H. Lomax, T. H. Pulliam, and D. W. Zingg. Fundamentals of computational fluid dynamics. Course Reader of AA214A Stanford University, 1998.

[69] R. P. Federenko. The Speed of Convergence of One Iterative Process. USSR Computational Mathematics and Mathematical Physics, 4:227-235, 1964.

[70] A. Jameson. Multigrid Algorithms for Compressible Flow Calculations. In Proceedings of the Second European Conference on Multigrid Methods, volume 1228, pages 166-201. Spring-Verlag, 1986.

[71] L. He. Method of Simulating Unsteady Turbomachinery Flows with Multiple Perturbations. AIAA Journal, 30(11):2730-2735, November 1992. 
[72] P. R. Spalart and S. R. Allmaras. A One -Equation Turbulence Model for Aerodynamic Flows. In Proceedings of 30th Aerospace Sciences Meeting and Exhibit, number AIAA-92-0439, January 1992.

[73] D. C. Wilcox. Turbulence Modeling for CFD. DCW Industries, 1993.

[74] B. A. Robinson, J. T. Batina, and H. T. Y. Yang. Aeroelastic Analysis of Wing Using the Euler Equations with a Deforming Mesh. Journal of Aircraft, 28(11):781-788, November 1991.

[75] J. J. Reuther, A. Jameson, J. Farmer, L. Martinelli, and D. Saunders. Aerodynamic Shape Optimization of Complex Aircraft Configurations via an Adjoint Formulation. In 34th Aerospace Sciences Meeting and Exhibit, number AIAA96-0094, January 1996.

[76] J. F. White and O. O. Bendiksen. Aeroelastic Behavior of Low-Aspect-Ratio Metal and Composite Blades. Journal of Engineering for Gas Turbines and Power, 109(2):168-175, April 1987.

[77] A. W. Leissa, J. K. Lee, and A. J. Wang. Rotating Blade Vibration Analysis Using Shells. Journal of Engineering for Power, 104(2):296-302, April 1982.

[78] J. S. Rao. Turbomachine Blade Vibration. John Wiley and Sons, 1991.

[79] M. Imregun. Structural Dynamics: Basics of Disk and Blade Vibration. In Aeroelasticity in Axial Flow Turbomachines, 1999-05. von Karman Institute, 1999.

[80] K. J. Bathe. Finite Element Procedures. Prentice Hall, 1996.

[81] J. S. Przemieniecki. Theory of Matrix Structural Analysis. McGraw-Hill, 1968.

[82] D. N. Herting. MSC/NASTRAN Advanced Dynamic Analysis User's Guide. MacNeal-Schwendler Corporation, 1997.

[83] O. G. McGee. Finite Element Analysis of Flexible, Rotating Blades. Technical Report NACA-TM-89906, July 1987. 
[84] R. E. Kielb and M. Imregun. Damping Characteristics. In Aeroelasticity in Axial-Flow Turbomachines, 1999-05. von Karman Institute, 1999.

[85] C. Lawrence, R. A. Aiello, and M. A. Ernst. A NASTRAN Primer for the Analysis of Rotating Flexible Blades. Technical Report NACA-TM-89861, May 1987.

[86] D. Bella and M. Reymond. MSC/NASTRAN DMAP Module Dictionary Version 68. MacNeal-Schwendler Corporation, 1995.

[87] M. G. Katona and O. C. Zienkiewicz. A Unified Set of Single Step Algorithms Part 3: The Beta-m Method, A Generalization of the Newmark Scheme. International Journal for Numerical Methods in Engineering, 21:1345-1359, February 1985 .

[88] G. Sitton. MSC/NASTRAN Basic Dynamic Analysis User's Guide. MacNealSchwendler Corporation, 1997.

[89] G. P. Guruswamy. Unsteady Aerodynamics and Aeroelastic Calculations for Wing Using Euler Equations. AIAA Journal, 28(3):461-469, March 1990.

[90] S. A. Brown. Displacement extrapolations for CFD+CSM aeroelastic analysis. In Proceedings of AIAA/ASME/ASCE/AHS/ASC Structures, Structural Dynamics, and Materials Conference and Exhibit, 38th, and AIAA/ASME/AHS Adaptive Structures Forum, number AIAA-97-1090, 1997.

[91] J. J. Reuther, J. J. Alonso, J. R. R. A. Martins, and S. C. Smith. A Coupled Aero-Structural Optimization Method For Complete Aircraft Configurations. In 37th Aerospace Sciences Meeting and Exhibit, number AIAA-99-0187, January 1999.

[92] C. Farhat and M. Lesoinne. Numerical Solutions of Euler Equation. In unknown, number AIAA-98-1806, 1998.

[93] J. Reuther, J. J. Alonso, J. C. Vassberg, A. Jameson, and L. Martinelli. An Efficient Multiblock Method for Aerodynamic Analysis and Design on Distributed 
Memory Systems. In AIAA Computational Fluid Dynamics Conference, number AIAA-97-1893, June 1997.

[94] R. V. Doggett, A. G. Rainey, and H. G. Morgan. An Experimental Investigation of Aerodynamic Effects of Airfoil Thickness on Transonic Flutter Characteristics. Technical Report NASA-TM-X-79, November 1959.

[95] J. J. Alonso. Parallel Computations of Unsteady and Aeroelastic Flow Using an Implicit Multigrid-Driven Algorithm. PhD thesis, Princeton University, 1997.

[96] D. C. Urasek, W. T. Gorrell, and W. S. Cunnan. Performance of Two Stage Fan Having Low-Aspect-Ratio, First-Stage Rotor Blading. Technical Report NACA-TP-1493, August 1979.

[97] R. V. Chima. Viscous Three-Dimensional Calculations of Transonic Fan Performance. Technical Report NASA-TM-103800, 1991.

[98] C. Hah and L. Reid. A Viscous Flow Study of Shock-Boundary Layer Interaction, Radial Transport, and Wake Development in a Transonic Compressor. Journal of Turbomachinery, 114(3):538-547, July 1992. ASME-91-GT-69.

[99] I. K. Jennions and M. G. Turner. Three-Dimensional Navier-Stokes Computations of Transonic Fan Flow Using an Explicit Flow Solver and an Implicit k- $\omega$ Solver. Journal of Turbomachinery, 115(1):261-272, April 1993.

[100] J. J. Adamczyk, M. L. Chelestina, and E. M. Greitzer. The Role of Tip Clearance in High-Speed Fan Stall. Journal of Turbomachinery, 115(1):28-39, January 1993.

[101] A. Arnone. Viscous Analysis of Three-Dimensional Rotor Flow Using a Multigrid Method. Journal of Turbomachinery, 116(3):435-445, July 1994.

[102] H. A. Chuang and J. M. Verdon. A Numerical Simulator for Three-Dimensional Flows Through Vibrating Blade Rows. Technical Report NASA-CR-1998208511, 1998. 
[103] R. E. Kielb. Forced Response Design Analysis. In Aeroelasticity in Axial-Flow Turbomachines, 1999-05. von Karman Institute, 1999. 